\title{
A Reduced-Order Model of Transport Phenomena for Power Plant Simulation
}

\author{
Final REPORT \\ Reporting Period Start Date: 06/17/05 \\ Reporting Period End Date: 09/17/09 \\ Principal Investigator: Paul Cizmas \\ Graduate Students: Brian Richardson, Thomas Brenner \\ AND RAYMOND FONTENOT \\ Date Report was Issued: OCtober 2009 \\ DOE Award Number: DE-FC26-05NT42445 \\ Texas Engineering Experiment Station \\ Office of Sponsored Research \\ 332 Wisenbaker Engineering Research Center, 3000 TAMU \\ College Station, TX 77843-3000
}




\section{Disclaimer}

This report was prepared as an account of work sponsored by an agency of the United States Government. Neither the United States Government nor any agency thereof, nor any of their employees, makes any warranty, express or implied, or assumes any legal liability or responsibility for the accuracy, completeness, or usefulness of any information, apparatus, product, or process disclosed, or represents that its use would not infringe privately owned rights. Reference herein to any specific commercial product, process, or service by trade name, trademark, manufacturer, or otherwise does not necessarily constitute or imply its endorsement, recommendation, or favoring by the United States Government or any agency thereof. The views and opinions of authors expressed herein do not necessarily state or reflect those of the United States Government or any agency thereof. 


\section{Abstract}

This report summarizes the objectives and tasks of this project, and describes how they were achieved. The report presents the progress made towards the program milestones and deliverables and summarizes the additional work done in the pursuit of these goals.

A reduced-order model based on proper orthogonal decomposition (POD) has been developed to simulate transient two- and three-dimensional isothermal and non-isothermal flows in a fluidized bed. Reduced-order models of void fraction, gas and solids temperatures, granular energy, and z-direction gas and solids velocity have been added to the previous version of the code. These algorithms are presented and their implementation is discussed. Verification studies are presented for each algorithm.

A number of methods to accelerate the computations performed by the reduced-order model are presented. The errors associated with each acceleration method are computed and discussed. Using a combination of acceleration methods, a two-dimensional isothermal simulation using the reduced-order model is shown to be 114 times faster than using the full-order model.

In the pursue of achieving the objectives of the project and completing the tasks planned for this program, several unplanned and unforeseen results, methods and studies have been generated. These additional accomplishments are also presented and they include: (1) a study of the effect of snapshot sampling time on the computation of the POD basis functions, (2) an investigation of different strategies for generating the autocorrelation matrix used to find the POD basis functions, (3) the development and implementation of a bubble detection and tracking algorithm based on mathematical morphology, (4) a method for augmenting the proper orthogonal decomposition to better capture flows with discontinuities, such as bubbles, and (5) a mixed reduced-order/full-order model, called point-mode proper orthogonal decomposition, designed to avoid unphysical due to approximation errors.

The limitations of the proper orthogonal decomposition method in simulating transient flows with moving discontinuities, such as bubbling flows, are discussed and several methods are proposed to adapt the method for future use. 


\section{List of Acronyms}

$\begin{array}{ll}\text { FOM } & \text { Full-Order Model } \\ \text { MFIX } & \text { Multiphase Flow with Interphase eXchanges (software) } \\ \text { ODEx } & \begin{array}{l}\text { Software for solving isothermal multiphase flow using Proper Orthogo- } \\ \text { nal Decomposition }\end{array} \\ \text { ODEt } & \begin{array}{l}\text { Software for solving non-isothermal multiphase flow using Proper Or- } \\ \text { thogonal Decomposition }\end{array} \\ \text { POD } & \text { Proper Orthogonal Decomposition } \\ \text { PODDEC } & \text { Proper Orthogonal Decomposition (software) } \\ \text { ROM } & \text { Reduced-Order Model } \\ \text { SVFCE } & \text { Solids Volume Fraction Correction Equation }\end{array}$




\section{Contents}

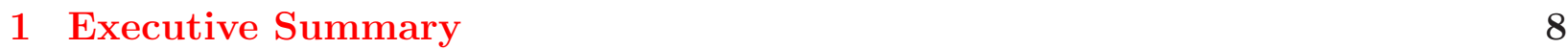

\begin{tabular}{lll}
\hline 2 & Introduction & 10
\end{tabular}

\begin{tabular}{|ccc}
\hline 3 & Experimental & 11
\end{tabular}

4 Results and Discussion 11

4.1 Program Deliverables . . . . . . . . . . . . . . . . . . . . . . . 11

4.1 .1 Database Generation for an Isothermal Flow . . . . . . . . . . . . . . 11

$4.1 .2 \quad$ Algorithm for Void Fraction . . . . . . . . . . . . . . . . . . . . . . . 13

4.1 .3 Void Fraction Algorithm Implementation . . . . . . . . . . . . . . . . 15

$4.1 .4 \quad$ Verification of Void Fraction Algorithm . . . . . . . . . . . . . . . . . 17

4.1 .5 Database Generation for a Non-Lsothermal Flow . . . . . . . . . . . . 25

4.1 .6 Reduced-Order Algorithm for Gas and Solids Temperatures . . . . . 26

4.1 .7 Implementation of the Energy Balance Equations in ODEt . . . . . . 31

1.1.8 $\quad$ Test, Verify and Validate ODEt . . . . . . . . . . . . . . . . . . 32

4.1 .9 Acceleration Methods for the ROM . . . . . . . . . . . . . . . . . . . 38

4.1 .10 CAPE-Open . . . . . . . . . . . . . . . . . . . . . . . . . 49

4.1 .11 User Manual and Theory Guidt . . . . . . . . . . . . . . . . . . . . . 50

4.1 .12 Interpolation and Extrapolation . . . . . . . . . . . . . . . . . . 50

4.1 .13 Dissemination of Research Results . . . . . . . . . . . . . . . . . . . . 53

4.1 .14 Granular Energy . . . . . . . . . . . . . . . . . . . . . 54

4.1 .15 Three-Dimensional Algorithm for ROM . . . . . . . . . . . . . . . . . 56

4.1 .16 3D Algorithm Implementation . . . . . . . . . . . . . . . . 57

4.1 .17 Testing of ROM vs. FOM . . . . . . . . . . . . . . . . . . . . 58

4.1 .18 3D Granular Energy and Temperature Algorithm . . . . . . . . . . . 58

4.2 Additional Accomplishments . . . . . . . . . . . . . . . . . . . . . . . 61

4.2 .1 Snapshot Distribution Study . . . . . . . . . . . . . . . . . . . . 61

4.2 .2 Composition of the Autocorrelation Matrix . . . . . . . . . . . . . . . 64

4.2 .3 Augmented Proper Orthogonal Decomposition . . . . . . . . . . . . . 67

4.2 .4 Point-Mode POD . . . . . . . . . . . . . . . . 76

$4.2 .5 \quad$ Morphology for Bubble Detection . . . . . . . . . . . . . . . . . . . . 79

$\begin{array}{llr}5 & \text { Conclusions } & 83\end{array}$

$\begin{array}{llr}6 & \text { Future Work } & 85\end{array}$

\section{List of Figures}

$1 \quad$ Geometry and boundary conditions for the two-dimensional fluidized bed. . . 14 
2 Time history of the first time coefficient calculated using MFIX, Hybrid and

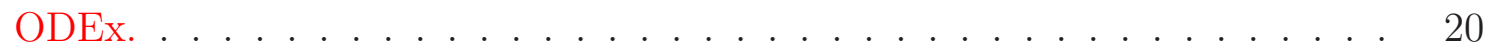

3 Time history of the second time coefficient calculated using MFIX, Hybrid

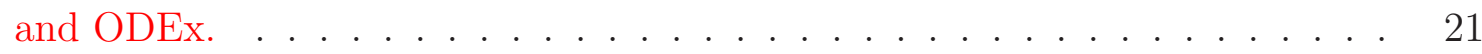

$4 \quad$ Contour plots at $t=1$ s showing gas pressure $\left(p_{q}\right)$ and void fraction $\left(\epsilon_{q}\right)$ using MFIX, Hybrid and ODEx. . . . . . . . . . . . . . . . . 22

5 Contour plots at $t=1 \mathrm{~s}$ showing $u$ and $v$ gas velocities using MFIX, Hybrid

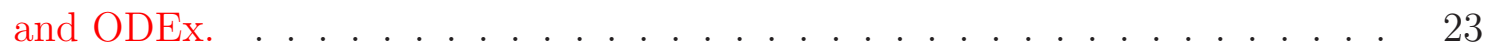

6 Contour plots at $t=1 \mathrm{~s}$ showing $u$ and $v$ solids velocities using MFTX, Hybrid

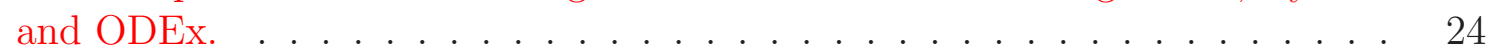

$7 \quad$ Average mode and first three basis functions of gas temperature, $T_{q}$. . . . . 26

$8 \quad$ Average mode and first three basis functions of solids temperature, $T_{s} . \quad$. . 26

9 Gas and solids temperature at $t=1.0 \mathrm{~s}:$ MFTX and ODEt. . . . . . . . . . . 35

10 Gas and solids temperature at $t=1.0 \mathrm{~s}$ in greater detail: MFIX and ODEt. . 36

11 Comparison of time coefficients for solids temperature: (a) $\alpha_{1}$, and (b) $\alpha_{2}$. 37

12 Comparison of time coefficients for solids temperature: (a) $\alpha_{1}^{T_{s}}$, and (b) $\alpha_{2}^{T_{s}} .37$

13 Cumulative energy spectrum for a database that spans $0.2-1.0 \mathrm{~s}$. . . . . . . . 39

14 Cumulative energy spectrum for a database that spans $0.2-0.35 \mathrm{~s}$. . . . . . . 39

15 Cumulative energy spectrum for a database that spans $0.35-1.0 \mathrm{~s}$. . . . . . . 39

16 Relationship between physical time and CPU time. . . . . . . . . . . . 43

17 Time step size vs. physical time: (a) FOM, and (b) ROM. . . . . . . . . . . 45

18 Gas pressure, void fraction and gas velocity $u_{g}$ at $t=1.5 \mathrm{~s}:$ MFIX and ODEx. 47

19 Gas velocities $v_{q}$ and solids velocities $\left(u_{s}, v_{s}\right)$ at $t=1.5 \mathrm{~s}:$ MFIX vs. ODEx. 48

20 Fourth basis function for void fraction. . . . . . . . . . . . . . . . 51

21 Fifth basis function for void fraction. . . . . . . . . . . . . . . . . . . . 51

22 Fourth time coefficient for void fraction. . . . . . . . . . . . . . . . . . 52

23 Fifth time coefficient for void fraction. . . . . . . . . . . . . . . . . . 52

$24 \quad$ FFT of fourth time coefficient for void fraction. . . . . . . . . . . . . . . . . 52

$25 \quad$ FFT of fifth time coefficient for void fraction. . . . . . . . . . . . . . . . 52

26 Contour plots of granular energy at $t=1 \mathrm{~s}:$ (a) MFIX, and (b) ODEx. . . . 56

$27 \quad$ Comparison of time coefficients for granular energy: (a) $\alpha_{1}^{\Theta_{m}}$, and (b) $\alpha_{2}^{\theta_{m}}$. . 57

28 Time history of the first time coefficient calculated using PODDEC and ODEX for the 3D test case . . . . . . . . . . . . . . . . . 59

29 Time history of the second time coefficient calculated using PODDEC and ODEx for the 3D test case. . . . . . . . . . . . . . . . . . 60

30 Methods for varying time between snapshots . . . . . . . . . . . . . . . . . 62

$31 \quad$ Relationship between physical time and CPU time in ODEx, with curve fit. . 62

32 Velocity profile at $t=1.0 \mathrm{~s} \ldots \ldots \ldots \ldots$. . . . . . . . . . . . . . . . . . . . . . . .

33 First mode time coefficients . . . . . . . . . . . . . . . . . . . 75

34 Discontinuity mode time coefficients . . . . . . . . . . . . . . . . . . 75

35 Contour plots of the average mode for $u_{q}$ : (a) unmasked, and (b) masked. . . 78

36 Contour plots indicating the location of two point modes: (a) $\phi_{10}^{\text {P1m }}$, and (b) $\phi_{18}^{\text {pmo }} 78$ 
37 Time histories for $u_{q}$ at two points in the flow, compared to: (a) $\alpha_{10}^{p m}$, and (b)

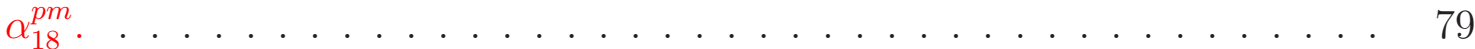

38 Contour plots for void fraction at $t=1.0$. . . . . . . . . . . . . . . . . 84

39 Shock detection algorithm results for a fluidized bed . . . . . . . . . . . . . . . 85

\section{List of Tables}

1 Program deliverables for project. . . . . . . . . . . . . . . . . . 12

2 Isothermal case parameters . . . . . . . . . . . . . . . . . . . . 13

$3 \quad$ Energy spectrum captured by the chosen number of modes. . . . . . . . . . . 17

$4 \quad$ Energy spectrum profile for the gas pressure and gas velocities. . . . . . . . . 19

$5 \quad$ Energy spectrum profile for the solids velocities and void fraction. . . . . . . 19

6 Non-isothermal case parameters . . . . . . . . . . . . . . . . 25

7 Most important routines created for ODEt. . . . . . . . . . . . . . . . . . 32

8 Most important routines modified for ODEt. . . . . . . . . . . . . . . . . . 33

$9 \quad$ Support routines modified for ODEt. . . . . . . . . . . . . . . . . . . . . . . 34

10 Number of modes used and their symbols. . . . . . . . . . . . . . . . . . . . 40

$11 \quad$ Eigenvalues during the transient and post transient regimes. . . . . . . . . . 43

$12 \quad$ Errors at $t=1.0 \mathrm{~s}$ with and without freezing. . . . . . . . . . . . . . . . . . 44

13 Speed-up factors and averaged errors: ROM vs. FOM . . . . . . . . . . . . 46

14 Interfaces identified for use in ODEX . . . . . . . . . . . . . . . . . . 49

15 Parameters of a bubbling fluidized bed (Gidaspow, 1994, p. 156). . . . . . . 53

16 POD reconstruction error using Equation (39) for various snapshot distributions 64

177 $\quad$ Number of modes used and their symbols. . . . . . . . . . . . . . . . . . . . 64

18 POD reconstruction error: split vs. coupled . . . . . . . . . . . . . . . . . 67

19 Parameters of fluidized bed Gidaspow (1994) . . . . . . . . . . . . . . . . . . . 83 


\section{Executive Summary}

The modeling of dense multiphase flow reactors is crucial for circulating fluidized-bed combustors and fluid catalytic cracking risers. The governing equations describing the phenomena inside multiphase flow reactors consist of a system of highly coupled partial differential equations. Solving these equations is a computationally intensive task. One goal of this project was to reduce the computational effort by improving a reduced-order model (ROM) code based on the proper orthogonal decomposition (POD) method. A second goal of this project was to implement a three-dimensional reduced-order algorithm that includes the conservation of energy and granular energy equations.

This report summarizes the objectives, tasks and accomplishments made during this research project. The report summarizes the project deliverables and details how the deliverables were achieved. It concludes with a discussion of additional accomplishments that were made in pursuit of the project objectives.

The first task, deriving, implementing and testing a reduced-order algorithm for computing the void fraction has been completed. A test case for two-dimensional isothermal flow was simulated using MFIX to build a database of snapshots. The POD basis functions were computed for this database and the ROM was shown to produce good results at reference conditions.

The second task, deriving, implementing and testing a reduced-order algorithm for the gas and solids temperatures has been completed. A test case for two-dimensional non-isothermal flow was simulated using MFIX to build a database of snapshots. The POD basis functions were computed, and the ROM was shown to produce good agreement with the full-order model (FOM) at reference conditions.

The third task, implementing an algorithm for granular energy has been completed. The granular energy has been modeled using both an algebraic equation and a reduced-order model of a partial differential equation. Both models are shown to be in good agreement with the FOM at reference conditions.

The fourth task, deriving, implementing and testing a reduced-order algorithm for the $z$-direction gas and solids velocities has been completed. The algorithms for the other field variables were updated to include the contributions of fluxes through the top and bottom faces of the computational cell. A three-dimensional isothermal test case was simulated using MFIX and a database of snapshot was collected. The POD basis functions computed from the database of snapshots were used in the ROM, which showed good agreement with MFIX at reference conditions.

Several acceleration methods for ROMs were developed and tested. These included a database splitting algorithm, an algorithm for solving quasi-symmetrical matrices and a strategy for updating some terms in the linear system of equations less often. The effect of time step adjustment was also explored. A combination of database splitting and initial time step adjustment was shown to give a speed-up factor of 114 .

The ROM was tested for cases of interpolation of particle diameter and fluid viscosity. It

was also used to extrapolate results in time, past the end of the database of snapshots. The 
POD time coefficients were studied in the frequency domain and useful information, such as the bubbling frequency, has been identified.

The interfaces necessary for the ROM to meet the CAPE-Open standards have been identified and summarized. Additionally a User's Manual and Theory Guide for ODEx have been written. These documents are described in this report.

Two practical aspects of POD have been studied and are presented in the additional accomplishments section. The first was the best placement of snapshots in time to minimize error. The second was the proper form of the autocorrelation matrix from which the POD basis functions are computed.

During the project, it was discovered that bubbles, which are moving discontinuities of the void fraction variable, cause the POD method to produce unphysical approximations for the flow when using a reduced number of modes. Several strategies for adapting the POD method to accurately model moving discontinuities are presented in the additional accomplishments section. Two of these methods, an augmented POD mode method and a point-mode method, have been implemented and tested for simple one-dimensional and two-dimensional canonical cases, such as the wave equation and Burgers equation. These methods have not been yet implemented in the ODEx code, but the initial results were promising and deserve further work. 


\section{Introduction}

This report summarizes the objectives, tasks and accomplishments made during this research project. The report presents the project deliverables and how they were achieved. Additional accomplishments made during the pursuit of the project objectives are also discussed.

A fourth year was added to this project as the project was ongoing. The additional deliverables that were added are summarized in the section detailing the program deliverables. All of the additional tasks have been completed.

The goal of this project was to add reduced-order models for the solids volume fraction correction equation, gas and solids energy balance, $z$-direction conservation of gas and solids momentum and the granular energy equation to ODEx, an existing reduced-order model for multiphase flow. These tasks have been completed and results of their verification are included.

During this project it was discovered that proper orthogonal decomposition (POD) based reduced-order models give unphysical approximations for field variables with moving discontinuities. This is a significant limitation, as it prevents the simulation of bubbling flow, where the bubbles are moving discontinuities in the void fraction. Two adaptations to the POD method are presented in the additional accomplishments section, a method for augmenting the POD basis functions and a point-mode approach. These two additional methods were

not fully implemented in the reduced-order model code, but both showed initial promise and deserve further work in the future. 


\section{$3 \quad$ Experimental}

No experimental work has been planned as part of this research project.

\section{Results and Discussion}

The purpose of this section is to present and discuss the progress made toward the program deliverables and additional accomplishments achieved in the process of reaching the program deliverables.

\subsection{Program Deliverables}

This section presents the work done in the specific areas of the program deliverables. The deliverables for this research project and their status is shown in Table $\mathbb{1}$.

\subsubsection{Database Generation for an Isothermal Flow}

The objective of producing a POD database for an isothermal, non-reactive fluidized bed has been achieved. The database was required to generate POD basis functions for the reduced-order model. The database was generated by first simulating a bubbling fluidized bed using MFIX. The basis functions were then extracted from the MFIX output data using the PODDEC code.

The parameters of the fluidized bed used for the database generation were taken from (Gidaspow, 1994, pp. 151-157). The control volume consisted of a rectangle discretized using 108 grid points in the $x$ direction and 124 grid points in the $y$ direction. In this particular case, the gas entered the lower boundary of the control volume with a constant velocity of $1 \mathrm{~cm} / \mathrm{s}$ in the vertical direction for the first 0.2 seconds. At 0.2 seconds the boundary conditions were changed to include a central jet with a velocity of $12.6 \mathrm{~cm} / \mathrm{s}$, which induced circulation in the bed. This calculation was performed from 0.2 to 1.0 seconds. These data were used to create the POD database. The calculations were split into two time periods (up to 0.2 seconds, and past 0.2 seconds) and only the second period was used to generate basis functions. This was done to avoid the transient flow that occurred when introducing the gas inflow into a completely stationary packed bed. The flow parameters are summarized in Table 2 and the geometry of the problem is shown in Fig. 1. 
Table 1: Program deliverables for project.

\begin{tabular}{|c|c|c|c|c|}
\hline & Program deliverable & Begin date & End date & Status \\
\hline 1. & Generate POD database for isothermal, non-reactive fluidized beds & $06 / 17 / 05$ & $9 / 18 / 05$ & Completed \\
\hline 2. & $\begin{array}{l}\text { Develop algorithm for reducing the solids volume fraction correction } \\
\text { equation }\end{array}$ & $9 / 18 / 05$ & $12 / 18 / 05$ & Completed \\
\hline 3. & $\begin{array}{l}\text { Begin implementation of the algorithm for the reduction of the SVFCE } \\
\text { in ODEx }\end{array}$ & $12 / 18 / 05$ & $02 / 24 / 06$ & Completed \\
\hline 4. & Test, verify and validate the fully-reduced POD model & $02 / 27 / 06$ & 09/16/06 & Completed \\
\hline 5. & $\begin{array}{l}\text { Generate database for non-isothermal, non-reactive fluidized beds and } \\
\text { calculate POD basis modes for these flows }\end{array}$ & $09 / 17 / 06$ & $12 / 16 / 06$ & Completed \\
\hline 6. & Develop algorithm for Galerkin projection of energy balance equations & $12 / 18 / 06$ & $03 / 16 / 07$ & Completed \\
\hline 7. & $\begin{array}{l}\text { Begin implementation of the algorithm for energy balance equations and } \\
\text { start developing the ODEt code }\end{array}$ & $03 / 17 / 07$ & $06 / 16 / 07$ & Completed \\
\hline 8. & Test, verify and validate ODEt against MFIX & $06 / 17 / 07$ & $09 / 16 / 07$ & Completed \\
\hline 9. & $\begin{array}{l}\text { Assess the accuracy and time efficiency (speed up) of the reduced-order } \\
\text { model }\end{array}$ & $09 / 17 / 07$ & $12 / 16 / 07$ & Completed \\
\hline 10. & Identify CAPE-OPEN standard interfaces & $12 / 17 / 07$ & $06 / 16 / 08$ & Completed \\
\hline 11. & Prepare User Manual and Theory Guide & $06 / 18 / 08$ & $07 / 01 / 08$ & Completed \\
\hline 12. & Evaluate the ROM vs. the FOM for interpolation and extrapolation cases & $07 / 02 / 08$ & $08 / 15 / 08$ & Completed \\
\hline 13. & Disseminate research results & $08 / 16 / 08$ & 09/07/08 & Completed \\
\hline 14. & Develop, implement and test algorithm for granular energy in the ROM & 09/08/08 & $11 / 01 / 08$ & Completed \\
\hline 15. & Develop algorithm for 3D ROM & $11 / 02 / 08$ & $12 / 15 / 08$ & Completed \\
\hline 16. & Implement 3D algorithm & $12 / 18 / 08$ & $03 / 22 / 09$ & Completed \\
\hline 17. & Test the $3 \mathrm{D}$ ROM vs. the FOM & 03/23/09 & $05 / 15 / 09$ & Completed \\
\hline 18. & Develop, implement and test 3D granular energy algorithm & $05 / 16 / 09$ & $06 / 15 / 09$ & Completed \\
\hline
\end{tabular}


Table 2: Isothermal case parameters

\begin{tabular}{llr}
\hline \hline Parameter & Description & Value \\
\hline$x_{\text {length }}$ & Length of the domain in $x$-direction & $25.4 \mathrm{~cm}$ \\
$y_{\text {length }}$ & Length of the domain in $y$-direction & $76.5 \mathrm{~cm}$ \\
imax & Number of cells in $x$-direction & 108 \\
jmax & Number of cells in $y$-direction & 124 \\
$v_{1}$ & Jet gas inflow velocity & $12.6 \mathrm{~cm} / \mathrm{s}$ \\
$v_{2}$ & Distributed gas inflow velocity & $1.0 \mathrm{~cm} / \mathrm{s}$ \\
$p_{s}$ & Static gas pressure at outlet & $1.01 \times 10^{6} \mathrm{~g} /\left(\mathrm{cm} \mathrm{s}^{2}\right)$ \\
$T_{g 0}$ & Gas temperature & $297 \mathrm{~K}$ \\
$\mu_{g 0}$ & Gas viscosity & $1.8 \times 10^{-4} \mathrm{~g} /(\mathrm{cm} \mathrm{s})$ \\
$t_{\text {start }}$ & Start time & $0 \mathrm{~s}$ \\
$t_{s t o p}$ & Stop time & $1 \mathrm{~s}$ \\
$\triangle t$ & Initial time step & $1.0 \times 10^{-4} \mathrm{~S}$ \\
$\rho_{s o}$ & Constant solids density & $1.0 \mathrm{~g} / \mathrm{cm}^{3}$ \\
$D_{p}$ & Solids particle diameter & $0.5 \mathrm{~mm}$ \\
$h_{s 0}$ & Initial packed bed height & $38.25 \mathrm{~cm}$ \\
$\epsilon_{g}^{*}$ & Packed bed void fraction & 0.40 \\
\hline
\end{tabular}

The PODDEC code was run on the MFIX output data. A total of twenty modes were extracted from each output variable. Each mode has 108 x $124=13392$ spatial components. The PODDEC code also generated twenty time coefficient files, each containing 320 time components, by projecting the MFIX snapshots onto the POD basis functions. These time coefficients were used to verify the results of the reduced-order model.

\subsubsection{Algorithm for Void Fraction}

The second deliverable of this project was the development of a reduced-order algorithm based on the solids volume fraction correction equation for calculating the void fraction. The methodology used in the full-order model (FOM), the MFIX code, approximates the solids volume fraction $\epsilon$ as

$$
\epsilon(x, t) \cong \epsilon^{*}(x, t)+\epsilon^{\prime}(x, t),
$$

where $\epsilon^{*}$ is a tentative value of $\epsilon$, i.e., $\epsilon^{*}$ was computed using old values of the state vector. $\epsilon^{\prime}$ is the correction of the tentative value. The approach used in MFIX to calculate the solids volume fraction is similar to the approach used to calculate pressure. As a result, the method developed herein for a reduced-order model of the solids volume fraction correction equation is similar to the method used for the reduced-order model of the pressure correction. 


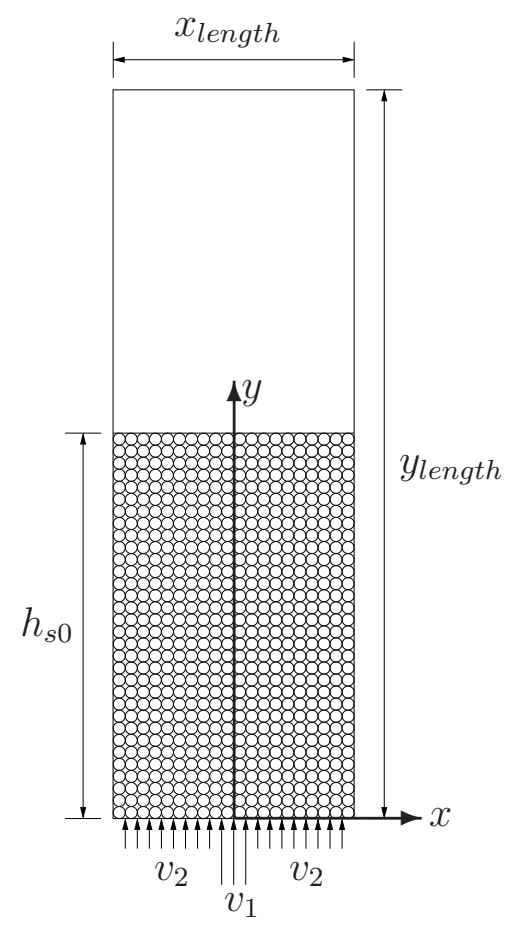

Figure 1: Geometry and boundary conditions for the two-dimensional fluidized bed.

The discretized form of the solids volume fraction correction equation is

$$
a_{p}^{\epsilon} \epsilon_{p}^{\prime}=\sum_{n b} a_{n b}^{\epsilon} \epsilon_{n b}^{\prime}+b_{p}^{\epsilon}
$$

where the $a_{n b}^{\epsilon}, a_{p}^{\epsilon}, b_{p}^{\epsilon}$ coefficients are defined in the MFIX Numerical Technique document (Syamla, 1998, pp. 48-52). The correction of the solids volume fraction is approximated using the basis functions of $\epsilon, \phi_{i}^{\epsilon}$

$$
\epsilon^{\prime}(x, t) \cong \sum_{j=1}^{m^{\epsilon}} \alpha_{j}^{\epsilon^{\prime}}(t) \phi_{j}^{\epsilon}(x),
$$

where $m^{\epsilon}$ is the number of solid volume fraction modes kept in the approximation. Note that the mean value of $\epsilon, \phi_{0}^{\epsilon}$, is not included in the series.

Substituting Equation (2) into Equation (11) yields

$$
a_{p}^{\epsilon} \sum_{j=1}^{m^{\epsilon}} \alpha_{j}^{\epsilon^{\prime}} \phi_{j_{p}}^{\epsilon}=\sum_{n b} a_{n b}^{\epsilon} \sum_{j=1}^{m^{\epsilon}} \alpha_{j}^{\epsilon^{\prime}} \phi_{j_{n b}}^{\epsilon}+b_{p}^{\epsilon}
$$


The Galerkin projection of Equation (3) onto the basis functions $\phi_{i}^{\epsilon}$ yields

$$
a_{p}^{\epsilon}\left(\sum_{j=1}^{m^{\epsilon}} \alpha_{j}^{\epsilon^{\prime}} \phi_{j_{p}}^{\epsilon}, \phi_{i}^{\epsilon}\right)=\sum_{n b} a_{n b}^{\epsilon}\left(\sum_{j=1}^{m^{\epsilon}} \alpha_{j}^{\epsilon^{\prime}} \phi_{j_{n b}}^{\epsilon}, \phi_{i}^{\epsilon}\right)+\left(b_{p}^{\epsilon}, \phi_{i}^{\epsilon}\right), \quad i=1, m^{\epsilon} .
$$

Equation (4) can be rearranged as

$$
\sum_{j=1}^{m^{\epsilon}} \alpha_{j}^{\epsilon^{\prime}}\left[a_{p}^{\epsilon}\left(\phi_{j_{p}}^{\epsilon}, \phi_{i}^{\epsilon}\right)-\sum_{n b} a_{n b}^{\epsilon}\left(\phi_{j_{n b}}^{\epsilon}, \phi_{i}^{\epsilon}\right)\right]=\left(b_{p}^{\epsilon}, \phi_{i}^{\epsilon}\right), \quad i=1, m^{\epsilon}
$$

The system of linear equations (5) can be written as

$$
\tilde{\mathcal{A}}^{\epsilon} \alpha^{\epsilon^{\prime}}=\tilde{\mathcal{B}}^{\epsilon}
$$

where

$$
\begin{aligned}
& \tilde{\mathcal{A}}_{i j}^{\epsilon}=\left(\left(a_{p}^{\epsilon} \phi_{j}^{\epsilon}-\sum_{n b} a_{n b}^{\epsilon} \phi_{j, n b}^{\epsilon}\right), \phi_{i}^{\epsilon}\right), \\
& \tilde{\mathcal{B}}_{i}^{\epsilon}=\left(b_{p}^{\epsilon}, \phi_{i}^{\epsilon}\right) .
\end{aligned}
$$

The dimensions of $\tilde{\mathcal{A}}$ and $\tilde{\mathcal{B}}$ are $m^{\epsilon} \times m^{\epsilon}$ and $m^{\epsilon} \times 1$, respectively.

An alternative method can be developed for deriving a reduced-order model of the solids volume fraction equation. This alternative method would use basis functions of the correction of the solids volume fraction, $\epsilon^{\prime}$, instead of the solids volume fraction, $\epsilon$. The approximation of the correction of the solids volume fraction would be

$$
\epsilon^{\prime}(x, t) \cong \phi_{0}^{\epsilon^{\prime}}(x)+\sum_{j=1}^{m^{\epsilon^{\prime}}} \alpha_{j}^{\epsilon^{\prime}}(t) \phi_{j}^{\epsilon^{\prime}}(x)
$$

where $\phi_{j}^{\epsilon^{\prime}}(x)$ are the basis functions of the correction of the solids volume fraction, and $m^{\epsilon^{\prime}}$ is the number of modes kept in the series.

\subsubsection{Void Fraction Algorithm Implementation}

The goal of implementing the algorithm to reduce the solids volume fraction correction equation into the ODEx code has been achieved. The task of implementing the reduced-order model (ROM) of the solids volume fraction correction equation into ODEx involved using the original equations as discretized in MFIX and projecting them onto the basis functions extracted using POD as described in the previous section. The implementation required the creation of two additional subroutines to be added to the existing Hybrid_puv ROM. Many additional modifications were made to existing subroutines during the implementation 
process. Below is a list of the primary subroutines modified or created during the completion of this task.

calc_ab_pp_s.f Projects the solids volume fraction correction equation on the basis functions. This routine uses the output from the conv_source_epp.f routine and the basis functions extracted from the flow snapshots to generate a linear system to represent the solids volume fraction correction equation.

reconstruct_ep_g.f Reconstructs the void fraction variable using the basis functions computed using POD and the time coefficients computed using ODEx.

solve_epp.f Primary control subroutine for the solution of the solids volume fraction correction equation. This routine was modified from Hybrid_puv to include the calc_ab_epp.f routine. The routine outputs the time coefficients of the solids volume fraction correction.

correct_1.f Applies the solids volume fraction correction to the solids velocities. This routine was modified from Hybrid_puv to use the time coefficients computed in the solve_epp.f subroutine.

Many additional modifications were made to supporting routines such as the module files, allocate_arrays.f, calc_resid.f and set_ic.f. 


\subsubsection{Verification of Void Fraction Algorithm}

The goal of testing, verifying and validating the fully-reduced POD model, which was implemented in the ODEx code, has been achieved. For the test case chosen, the number of modes used were $\mathrm{nP}_{-} \mathrm{g}=2, \mathrm{nU}_{-} \mathrm{g}=2, \mathrm{nV}_{-} \mathrm{g}=5, \mathrm{nU}_{-} \mathrm{s}=8, \mathrm{nV}_{-} \mathrm{s}=6, \mathrm{nEP}_{-} \mathrm{g}=7$. Table 3 shows the energy spectrum captured by the chosen variables for each of the six state variables. Tables $⿴$

\begin{tabular}{|c|c|c|}
\hline \hline Variable & \# Modes & \% Spectrum \\
\hline P_g & 2 & $99.997 \%$ \\
\hline U_g & 2 & $96.377 \%$ \\
\hline V_g & 5 & $99.972 \%$ \\
\hline U_s & 8 & $99.970 \%$ \\
\hline V_s & 6 & $99.989 \%$ \\
\hline EP_g & 7 & $99.999 \%$ \\
\hline \hline
\end{tabular}

Table 3: Energy spectrum captured by the chosen number of modes.

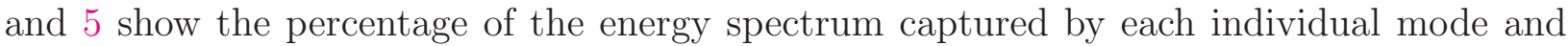
the cumulative energy captured by the sum over the modes for each field variable.

The gas pressure and gas velocity variables can be represented by only a few modes. The solids velocities and void fraction parameters require more modes to capture because the flow features for the chosen case are more complex and have more subtle velocity changes than the gas parameters. Using this test case it was possible to demonstrate that the new ODEx code was more computationally efficient than the previous ROM Hybrid_puv and MFIX. The required computation time for MFIX was 9761.453 seconds, for Hybrid_puv 3740.247 seconds and for ODEx 894.317 seconds. The reduced order ODEx code was able to run 11 times faster than the full numerical model, MFIX, and 4 times faster than the previous ROM, Hybrid_puv. Figures 2 [ 3 show time histories of the first two time coefficients computed for each state variable by ODEx, where they are compared to the best-case solution for the time coefficients computed by PODDEC and the results from Hybrid_puv.

The time histories show that the first two time coefficients computed by ODEx match match well to the 'exact' solution computed by PODDEC for the six field variables. The $y$ velocities time coefficients computed by Hybrid_puv are unusually poor for the first time coefficient but much better for the second time coefficient.

Figures 囵 compare the reconstructed field variables at $t=1.0 \mathrm{~s}$ to the MFIX solution. The contour plots show good correlation between the flow features computed by the full numerical model MFIX and the reduced-order models. The lone exception can be found in the solids $v$ velocity computed by ODEx. ODEx correctly computes the solids $v$ velocity except for the central region of the bed. The difference between the contour levels of the solids $v$ velocity, however, is extremely small. The entire solids $v$ velocity variation spans the interval $(-0.003,0.0002) \mathrm{cm} / \mathrm{s}$ while the gas $v$ velocity spans a much larger interval $(0.0$, 
$15.0) \mathrm{cm} / \mathrm{s}$.

The error of the solids $v$ velocity increases when the number of modes used for any of the solids parameters decreases. The accuracy of the solids $v$ velocity is particularly sensitive to changes of the number of modes used for the void fraction. This dependence on additional state variables makes it difficult to achieve greater computational efficiency by simply reducing the number of modes used to represent the solids parameters. For example, the number of modes of solids $u$ velocity, nU_s, could be reduced from its current value of 8 to 3 modes and still obtain good results for the solids $u$ velocities. However, the accuracy of the solution of the solids $v$ velocities would decrease. 


\begin{tabular}{|c|c|c|c|c|c|c|}
\hline \hline \multirow{2}{*}{$\begin{array}{c}\text { Number } \\
\text { of Modes }\end{array}$} & \multicolumn{2}{|c|}{ P_g } & \multicolumn{2}{c|}{ U_g $^{\prime}$} & \multicolumn{2}{c|}{ V_g } \\
\cline { 2 - 7 } & Energy\% & Total\% & Energy\% & Total\% & Energy\% & Total\% \\
\hline 1 & 99.938 & 99.938 & 90.102 & 90.102 & 85.018 & 85.018 \\
\hline 2 & 0.589 & 99.997 & 6.274 & 96.377 & 13.646 & 98.664 \\
\hline 3 & 0.024 & 99.999 & 2.914 & 99.291 & 0.986 & 99.650 \\
\hline 4 & 0.004 & 99.999 & 0.305 & 99.596 & 0.248 & 99.898 \\
\hline 5 & - & 99.999 & 0.220 & 99.815 & 0.074 & 99.972 \\
\hline 6 & - & 99.999 & 0.120 & 99.993 & 0.013 & 99.985 \\
\hline 7 & - & 99.999 & 0.026 & 99.996 & 0.008 & 99.993 \\
\hline 8 & - & 99.999 & 0.017 & 99.997 & 0.004 & 99.997 \\
\hline
\end{tabular}

Table 4: Energy spectrum profile for the gas pressure and gas velocities.

\begin{tabular}{|c|c|c|c|c|c|c|}
\hline \hline \multirow{2}{*}{$\begin{array}{c}\text { Number } \\
\text { of Modes }\end{array}$} & \multicolumn{2}{|c|}{ U_s } & \multicolumn{2}{c|}{ V_s } & \multicolumn{2}{c|}{ EP_g } \\
\cline { 2 - 7 } & Energy\% & Total\% & Energy\% & Total\% & Energy\% & Total\% \\
\hline 1 & 80.644 & 80.644 & 96.559 & 96.559 & 68.597 & 68.597 \\
\hline 2 & 15.373 & 96.017 & 2.018 & 98.577 & 27.541 & 96.138 \\
\hline 3 & 1.818 & 97.835 & 1.069 & 99.647 & 3.691 & 99.829 \\
\hline 4 & 1.252 & 99.086 & 0.203 & 99.850 & 0.112 & 99.941 \\
\hline 5 & 0.495 & 99.582 & 0.121 & 99.971 & 0.043 & 99.983 \\
\hline 6 & 0.223 & 99.804 & 0.018 & 99.989 & 0.014 & 99.998 \\
\hline 7 & 0.098 & 99.902 & 0.007 & 99.996 & 0.001 & 99.999 \\
\hline 8 & 0.068 & 99.970 & 0.002 & 99.999 & - & 99.999 \\
\hline
\end{tabular}

Table 5: Energy spectrum profile for the solids velocities and void fraction. 
EP_g Time Coefficient 1 vs. Time

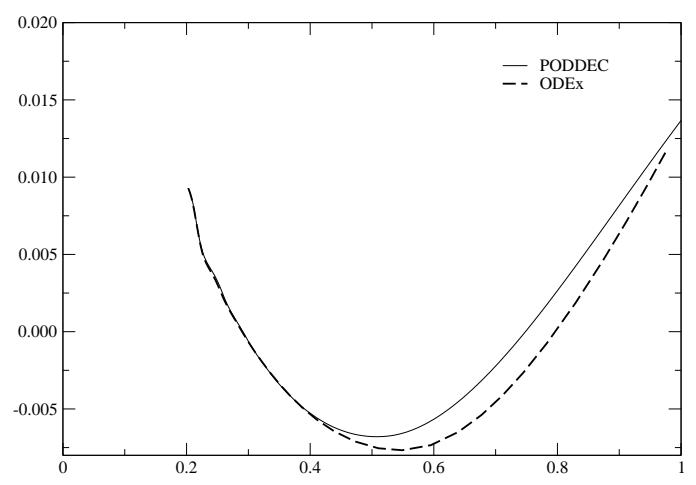

U_g Time Coefficient 1 vs. Time

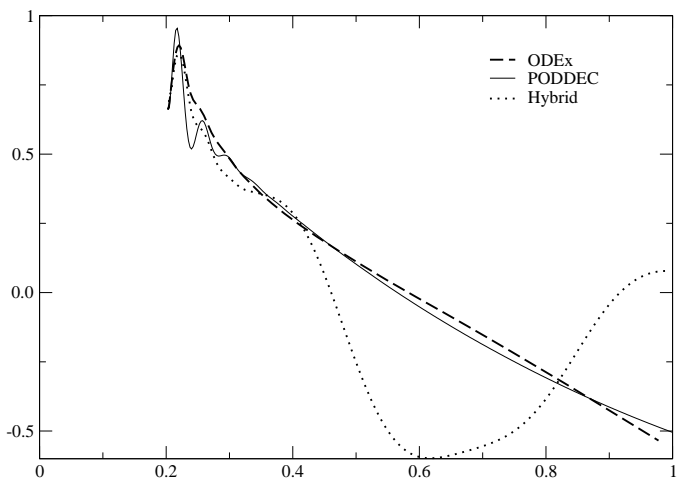

V_g Time Coefficient 1 vs. Time

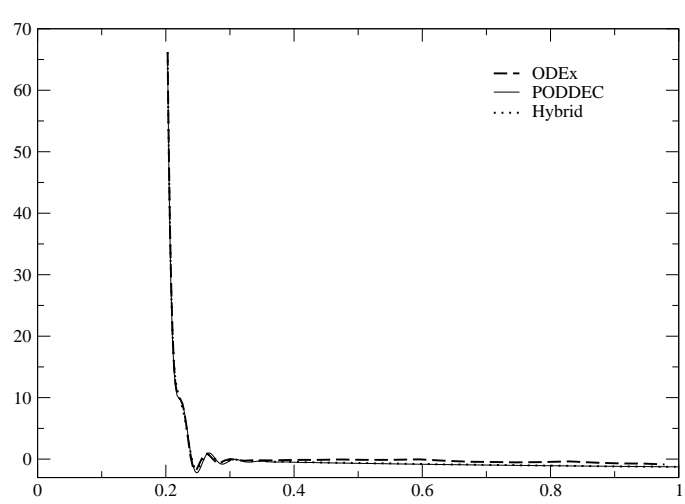

P_g Time Coefficient 1 vs. Time

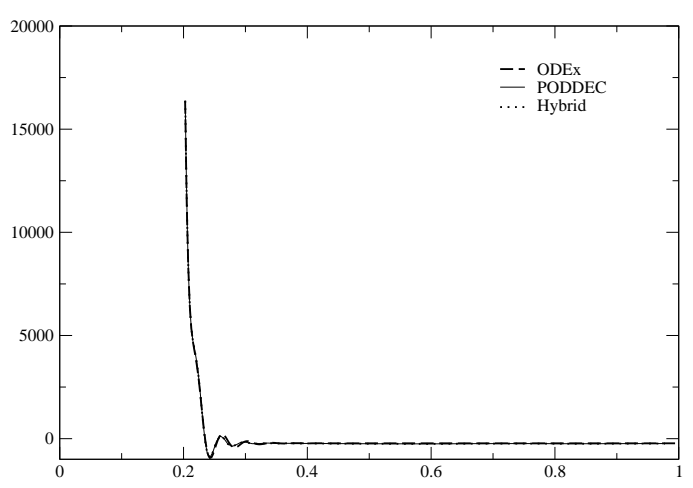

U_s Time Coefficient 1 vs. Time

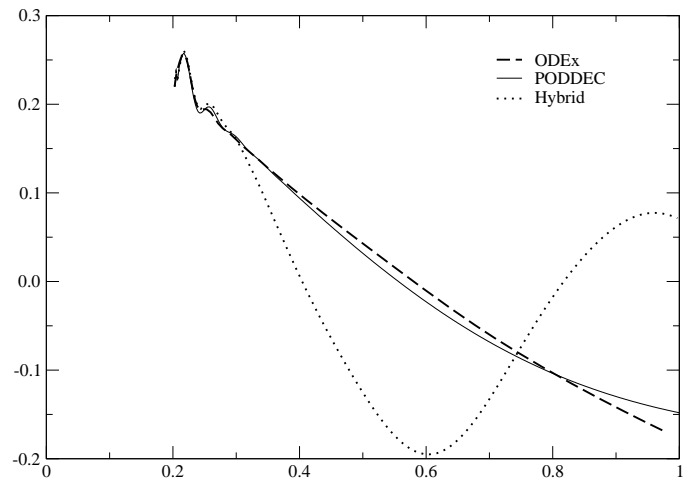

V_s Time Coefficient 1 vs. Time

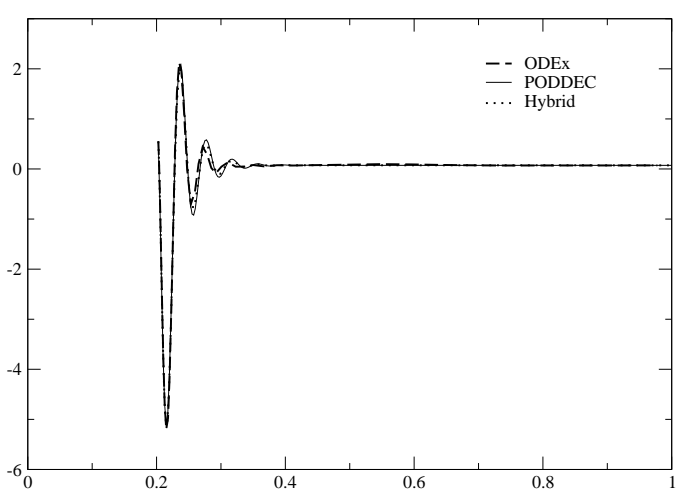

Figure 2: Time history of the first time coefficient calculated using MFIX, Hybrid and ODEx. 

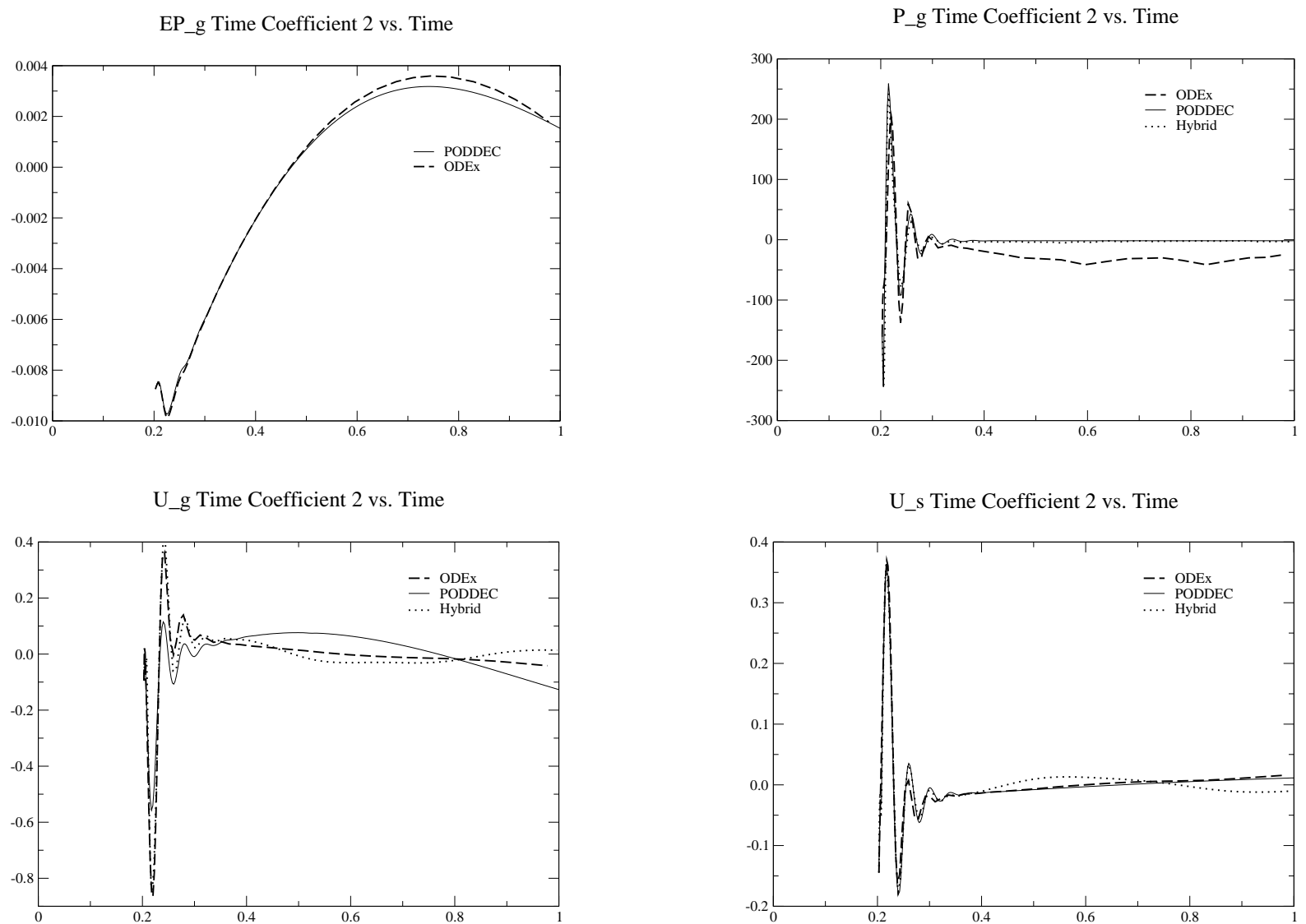

V_g Time Coefficient 2 vs. Time

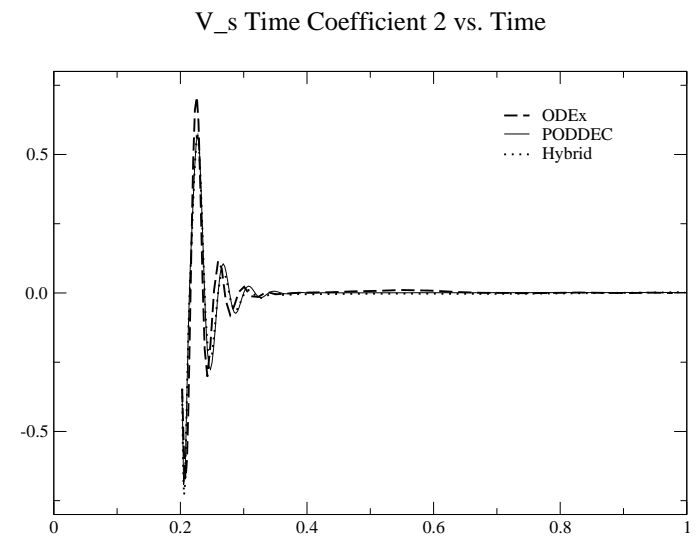

Figure 3: Time history of the second time coefficient calculated using MFIX, Hybrid and ODEx. 


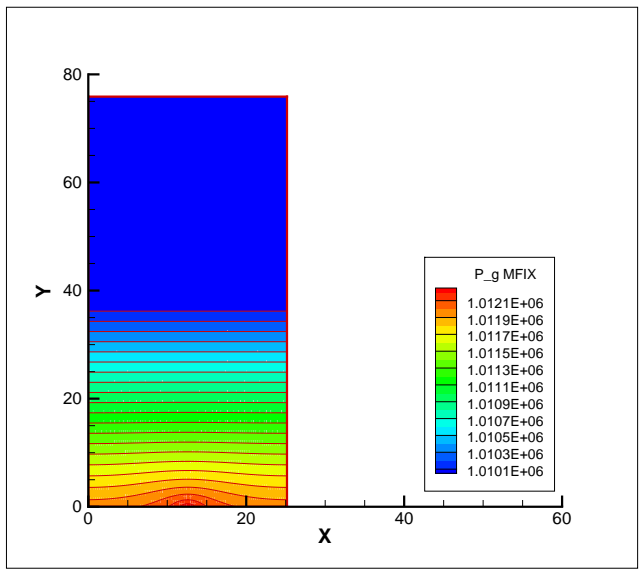

(a) MFIX gas pressure

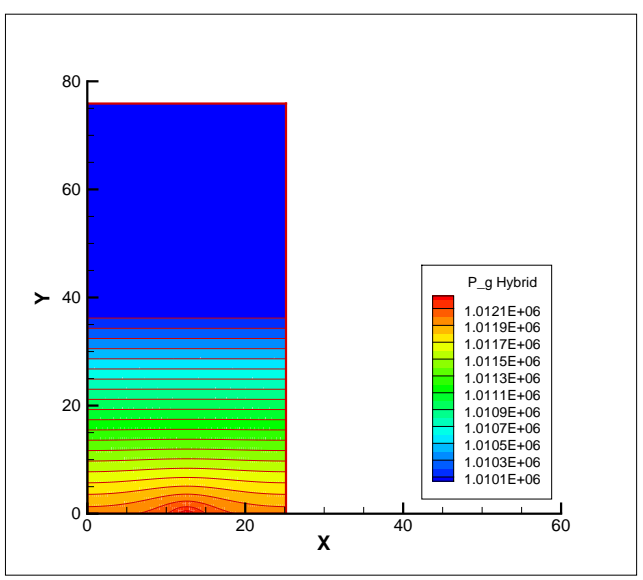

(c) Hybrid gas pressure

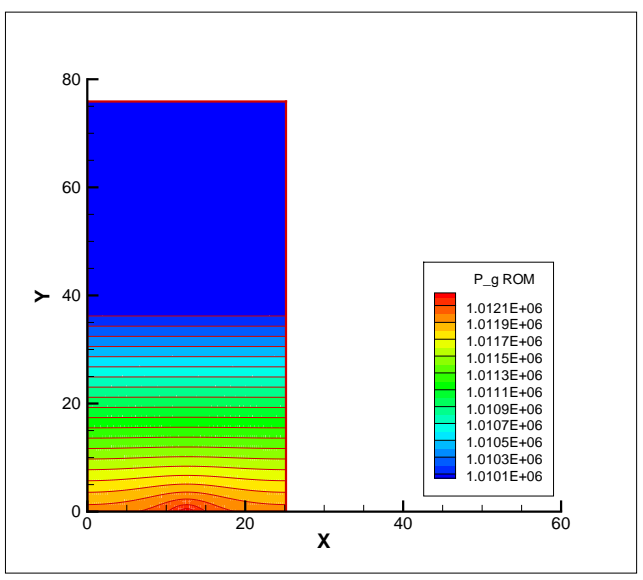

(d) ODEx gas pressure

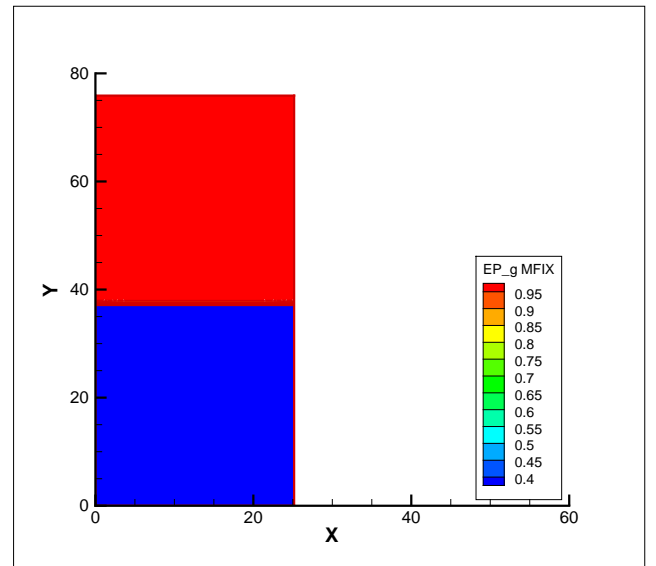

(b) MFIX void fraction

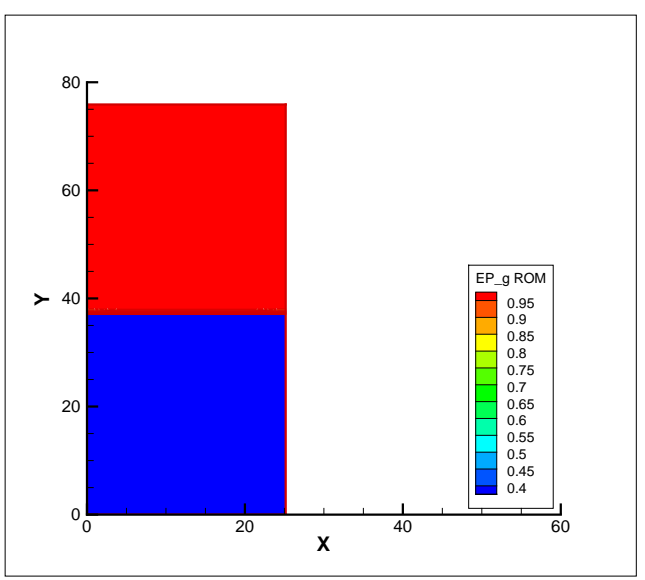

(e) ODEx void fraction

Figure 4: Contour plots at $t=1 \mathrm{~s}$ showing gas pressure $\left(p_{g}\right)$ and void fraction $\left(\epsilon_{g}\right)$ using MFIX, Hybrid and ODEx. 


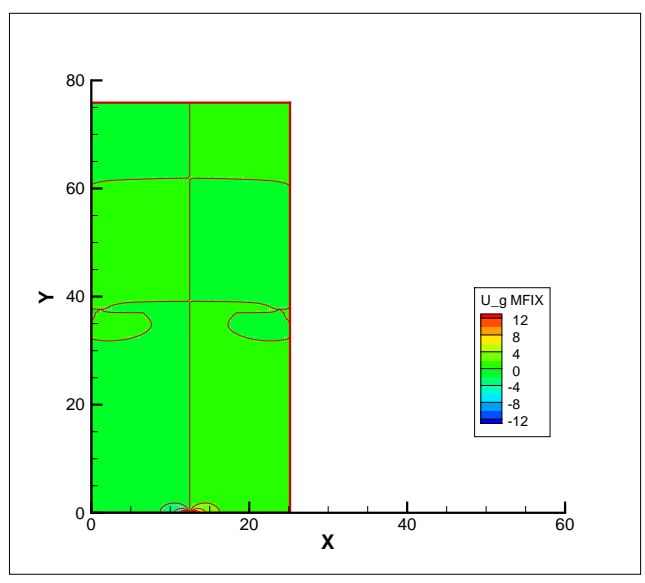

(a) MFIX $x$-direction gas velocity

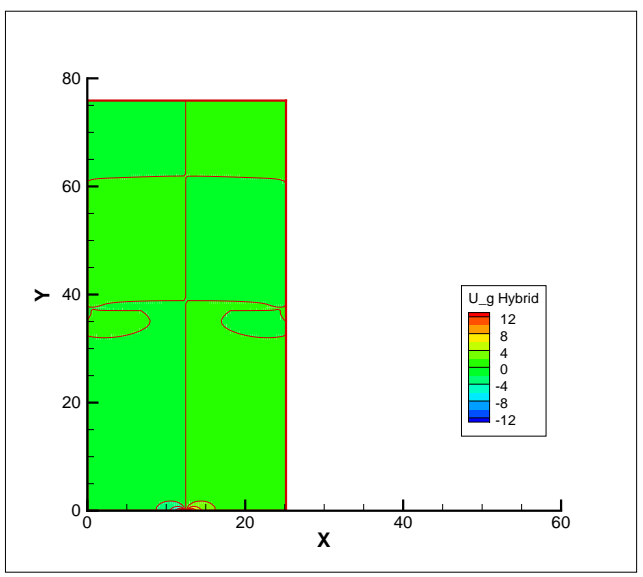

(c) Hybrid $x$-direction gas velocity

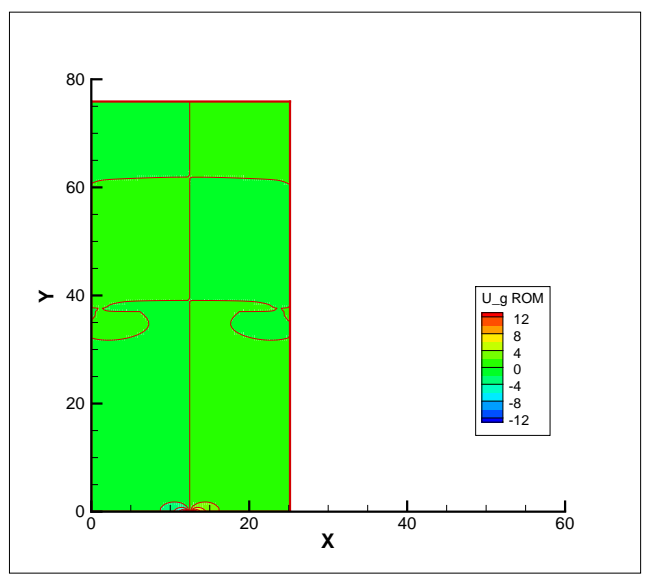

(e) ODEx $x$-direction gas velocity

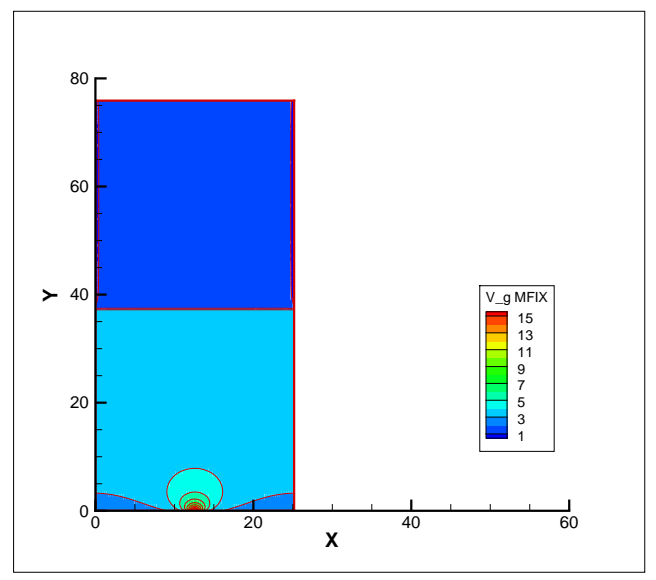

(b) MFIX $y$-direction gas velocity

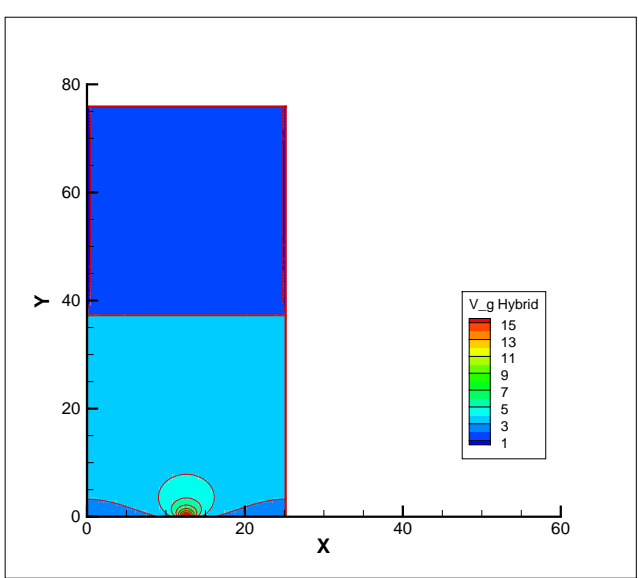

(d) Hybrid $y$-direction gas velocity

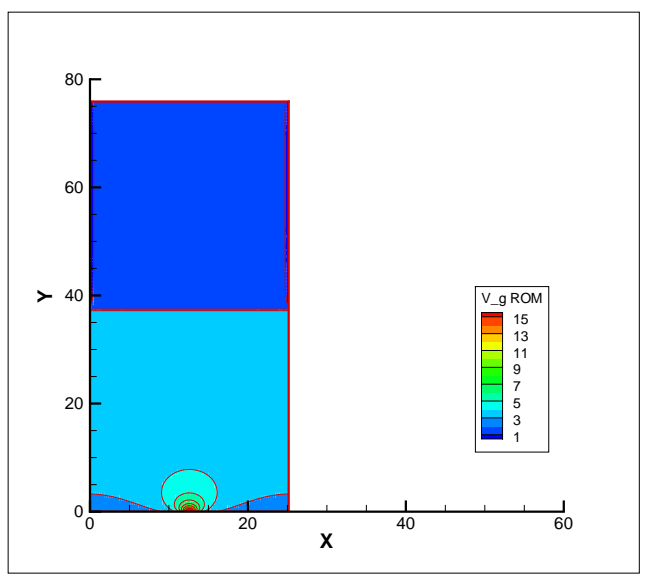

(f) ODEx $y$-direction gas velocity

Figure 5: Contour plots at $t=1 \mathrm{~s}$ showing $u$ and $v$ gas velocities using MFIX, Hybrid and ODEx. 


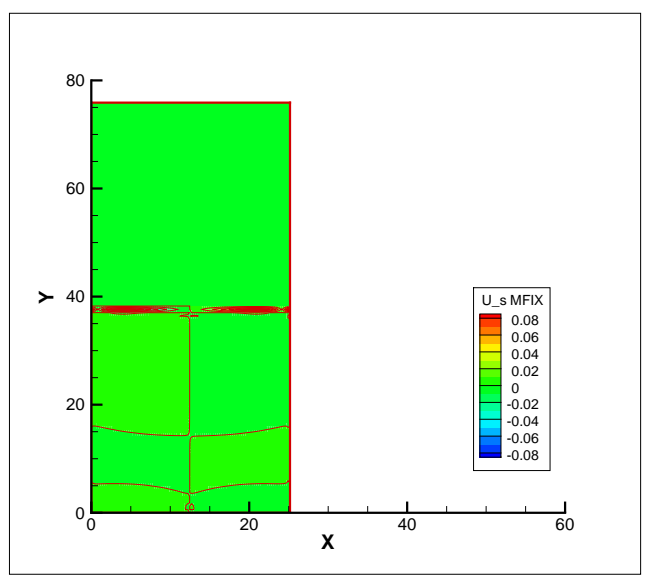

(a) MFIX $x$-direction solids velocity

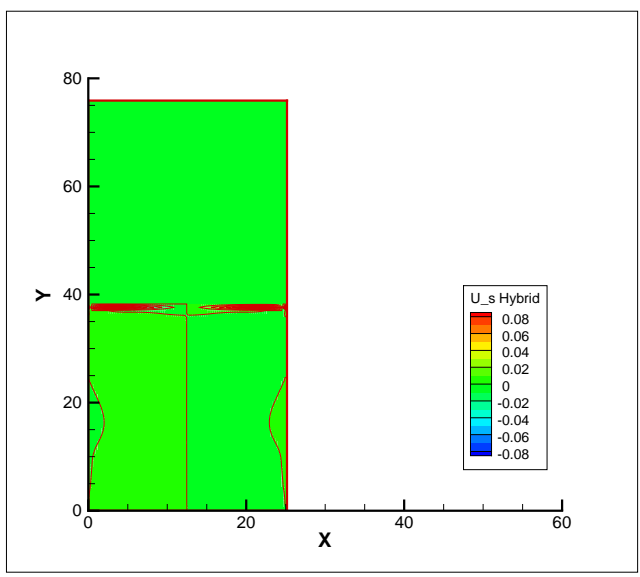

(c) Hybrid $x$-direction solids velocity

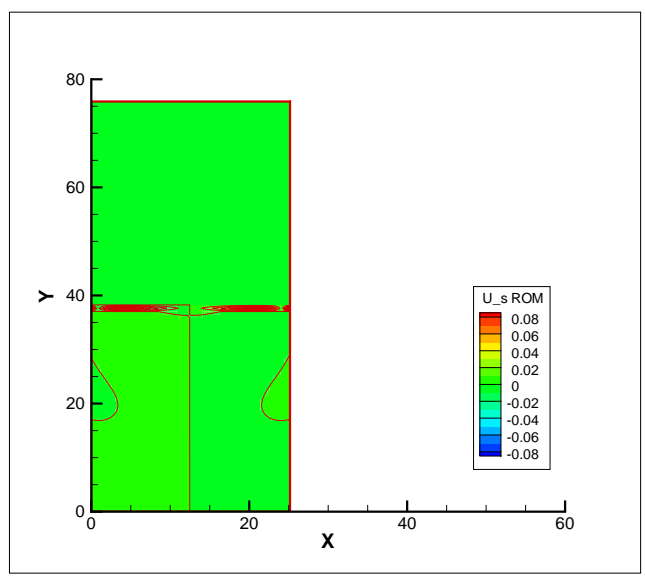

(e) ODEx $x$-direction solids velocity

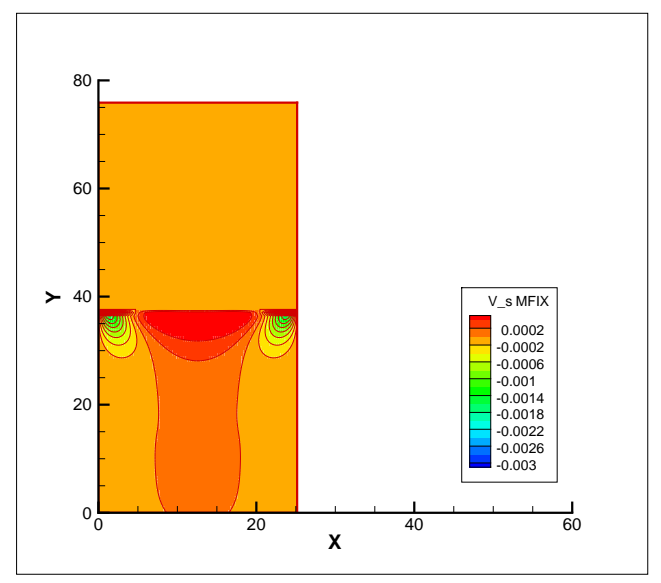

(b) MFIX $y$-direction solids velocity

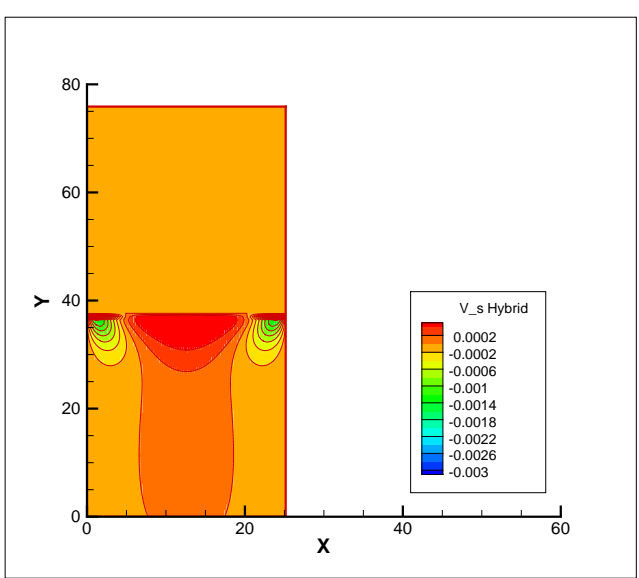

(d) Hybrid $y$-direction solids velocity

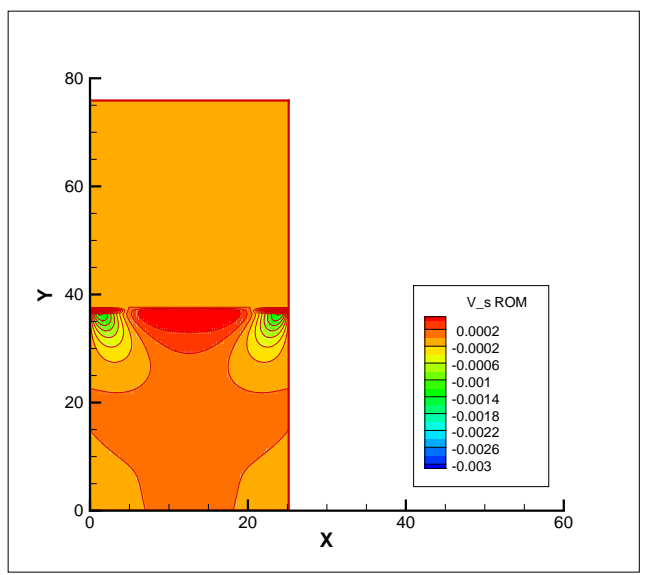

(f) ODEx $y$-direction solids velocity

Figure 6: Contour plots at $t=1 \mathrm{~s}$ showing $u$ and $v$ solids velocities using MFIX, Hybrid and ODEx. 


\subsubsection{Database Generation for a Non-Isothermal Flow}

A database for non-isothermal, non-reactive fluidized beds was generated and the POD basis functions were computed. The input parameters used in MFIX for the non-isothermal case are shown in Table 6 .

Table 6: Non-isothermal case parameters

\begin{tabular}{llr}
\hline \hline Parameter & Description & Value \\
\hline$x_{\text {length }}$ & Length of the domain in $x$-direction & $25.4 \mathrm{~cm}$ \\
$y_{\text {length }}$ & Length of the domain in y-direction & $76.5 \mathrm{~cm}$ \\
$i$ max & Number of cells in $x$-direction & 108 \\
$j$ max & Number of cells in $y$-direction & 124 \\
$v_{1}$ & Jet gas inflow velocity & $12.6 \mathrm{~cm} / \mathrm{s}$ \\
$v_{2}$ & Distributed gas inflow velocity & $1.0 \mathrm{~cm} / \mathrm{s}$ \\
$p_{s}$ & Static gas pressure at outlet & $1.01 \times 10^{6} \mathrm{~g} /\left(\mathrm{cm} \cdot \mathrm{s}^{2}\right)$ \\
$T_{g 0}$ & Initial gas temperature & $297 \mathrm{~K}$ \\
$T_{s 0}$ & Initial solids temperature & $297 \mathrm{~K}$ \\
$T_{g 1}$ & Jet gas inlet temperature & $450 \mathrm{~K}$ \\
$T_{g 2}$ & Distributed gas inlet temperature & $297 \mathrm{~K}$ \\
$\mu_{g 0}$ & Gas viscosity & $\mathrm{g} /(\mathrm{cm} \cdot \mathrm{s})$ \\
$t_{s t a r t}$ & Start time & $0 \mathrm{~s}$ \\
$t_{s t o p}$ & Stop time & $1 \mathrm{~s}$ \\
$\triangle t$ & Initial time step & $1.8 \times 10^{-4}$ \\
$\rho_{s o}$ & Constant solids density & $1.0 \mathrm{~g} / \mathrm{cm}^{3}$ \\
$D_{p}$ & Solids particle diameter & $0.5 \mathrm{~mm}$ \\
$h_{s 0}$ & Initial packed bed height & $38.25 \mathrm{~cm}$ \\
$C_{p g 0}$ & Initial gas phase specific heat & $0.25 \mathrm{cal} / \mathrm{gK}$ \\
$C_{p s 0}$ & Initial solids phase specific heat & $0.310713 \mathrm{cal} / \mathrm{gK}$ \\
$\epsilon_{g}^{*}$ & Packed bed void fraction & 0.40 \\
\hline & &
\end{tabular}

The input parameters for the non-isothermal case were similar to the input parameters used for the isothermal case given in Table 2, except for specifying that the jet inlet gas was 150 degrees hotter than the initial gas and solids phases in the control volume or the distributed flow. The control volume consisted of a rectangle discretized using 108 equidistant nodes in the $x$ direction and 124 equidistant nodes in the $y$ direction. In this particular case, the gas entered through the lower boundary of the control volume with a constant velocity of $1 \mathrm{~cm} / \mathrm{s}$ in the vertical direction for the first 0.2 seconds. At time $t=0.2$ seconds, the boundary conditions were changed and a centralized jet with a velocity of $12.6 \mathrm{~cm} / \mathrm{s}$ was specified in order to induce circulation in the bed. The test case geometry is shown in Fig. 1.

The calculations performed from 0.2 to 1.0 seconds were used to create the POD database. 
The calculations prior to $t=0.2$ seconds were discarded to avoid the transience that occurred when introducing the gas inflow into a completely stationary packed bed.

The database of snapshots generated by using MFIX was needed to extract the POD basis functions. The PODDEC code was used to extract the POD basis functions. Twenty modes were extracted for each of the state variables, that is, gas pressure, gas void fraction, $x$ - and $y$-direction gas and solids velocities, and gas and solids temperature. Contour plots of the average mode and first three basis functions of the gas and solids phase temperatures are shown in Figs. 0 and 8 .

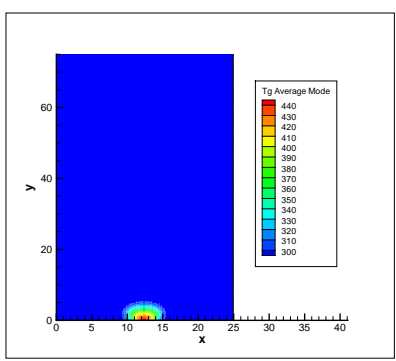

(a) $\phi_{0}^{T_{g}}$

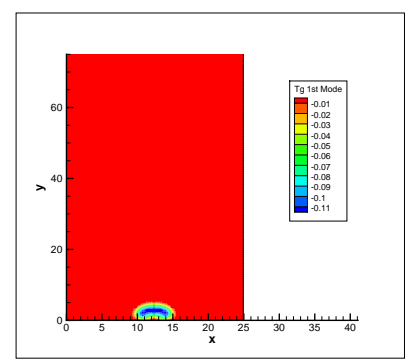

(b) $\phi_{1}^{T_{g}}$

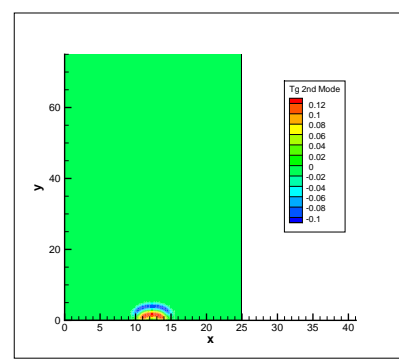

(c) $\phi_{2}^{T_{g}}$

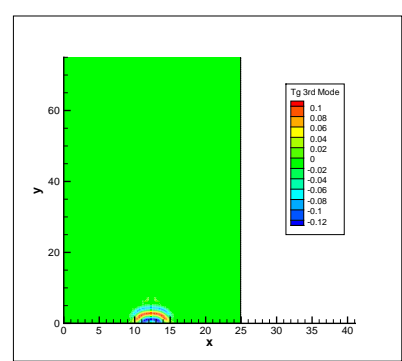

(d) $\phi_{3}^{T_{g}}$

Figure 7: Average mode and first three basis functions of gas temperature, $T_{g}$.

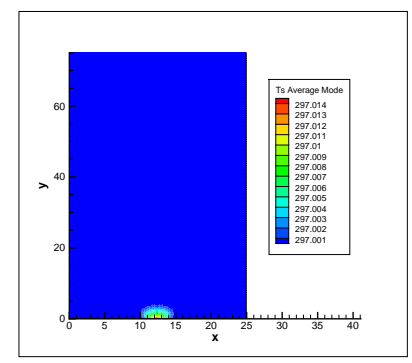

(a) $\phi_{0}^{T_{s}}$

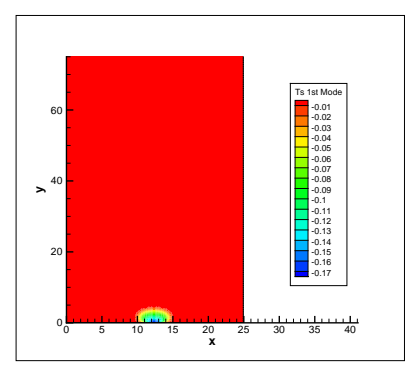

(b) $\phi_{1}^{T_{s}}$

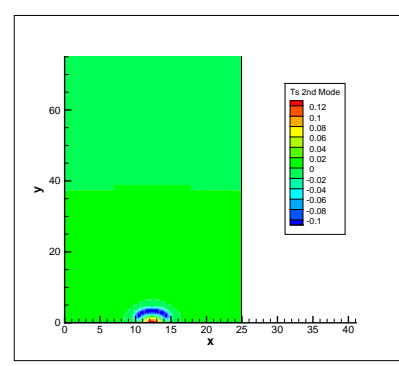

(c) $\phi_{2}^{T_{s}}$

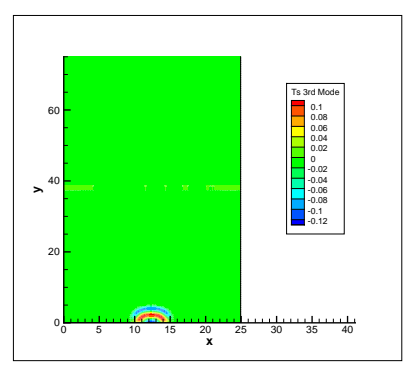

(d) $\phi_{3}^{T_{s}}$

Figure 8: Average mode and first three basis functions of solids temperature, $T_{s}$.

\subsubsection{Reduced-Order Algorithm for Gas and Solids Temperatures}

The scalar energy balance equations used to model the gas and solids phase temperature fields for a non-isothermal fluidized bed are (Syamla, [998, p. 62):

- Energy balance for fluids phase

$$
\epsilon_{g} \rho_{g} C_{p g}\left(\frac{\partial T_{g}}{\partial t}+\vec{v}_{g} \cdot \nabla T_{g}\right)=-\nabla \cdot \vec{q}_{g}+\gamma_{g \ell}\left(T_{s}-T_{g}\right)-\triangle H_{r g}+\gamma_{R g}\left(T_{R g}^{4}-T_{g}^{4}\right)
$$


- Energy balance for solids phase

$$
\epsilon_{s} \rho_{s} C_{p s}\left(\frac{\partial T_{s}}{\partial t}+\vec{v}_{s} \cdot \nabla T_{s}\right)=-\nabla \cdot \vec{q}_{s}-\gamma_{g \ell}\left(T_{s}-T_{g}\right)-\triangle H_{r s}+\gamma_{R s}\left(T_{R s}^{4}-T_{s}^{4}\right)
$$

where $\vec{q}_{g}, \triangle H_{r g}, \gamma_{g \ell}$ and $\gamma_{R g}$ represent the gas-phase conductive heat flux, heat of reaction, gas-solids heat transfer coefficient and fluids phase radiative heat transfer coefficient, respectively. Similar terms exist for the energy balance equation for solids phase.

The energy balance equations (17) and (8) can be written in the general form of transport equations. Consequently, the discretized energy equation is similar to the discretized scalar transport Equation (Syamla, 1998, p. 54). The discretized transport equation for a scalar, $\psi$, can be written as (Syamla], 1998, p. 18, Eq. 3.12)

$$
a_{P}(\psi)_{P}=\sum_{n b} a_{n b}(\psi)_{n b}+b_{P}
$$

where $p$ and $n b$ denote the cell center and neighbor cell centers, respectively. For the energy balance equation $\psi=T$, where $T$ can be the gas or solids phase temperature. The coefficients of the discretized energy balance equation were derived as

$$
\begin{aligned}
& a_{E}^{T_{\ell}} \quad=D_{e}-\frac{\left(\xi_{\ell}^{T}\right)_{e}}{2}\left(\epsilon_{\ell} \rho_{\ell}\right)_{E}\left(C_{p_{\ell}}+C_{p_{\ell E}}\right)\left(u_{\ell}\right)_{e} A_{e}, \\
& a_{W}^{T_{\ell}} \quad=D_{w}+\frac{\left(\bar{\xi}_{\ell}^{T}\right)_{w}}{2}\left(\epsilon_{\ell} \rho_{\ell}\right)_{W}\left(C_{p_{\ell}}+C_{p_{\ell E}}\right)\left(u_{\ell}\right)_{w} A_{w} \\
& a_{N}^{T_{\ell}} \quad=D_{n}-\frac{\left(\xi_{\ell}^{T}\right)_{n}}{2}\left(\epsilon_{\ell} \rho_{\ell}\right)_{N}\left(C_{p_{\ell}}+C_{p_{\ell N}}\right)\left(v_{\ell}\right)_{n} A_{n}, \\
& a_{S}^{T_{\ell}} \quad=D_{s}+\frac{\left(\bar{\xi}_{\ell}^{T}\right)_{s}}{2}\left(\epsilon_{\ell} \rho_{\ell}\right)_{S}\left(C_{p_{\ell}}+C_{p_{\ell N}}\right)\left(v_{\ell}\right)_{s} A_{s}, \\
& a_{p}^{T_{\ell}} \quad=-\left(\sum_{n b}\left(a_{\ell}^{T}\right)_{n b}+\frac{\left(\rho_{\ell}^{o}\right) C_{p_{\ell}} \Delta V}{\Delta t}+\gamma_{R m}\left(T_{\ell}^{o}\right)^{3}\right), \quad \text { (Ge) } \\
& \left.b_{p}^{T_{\ell}} \quad=-\left(\frac{\left(\rho_{\ell}^{o}\right) C_{p_{\ell}} \Delta V}{\triangle t}\left(T_{\ell}^{o}\right)-\triangle H_{R m} \triangle V+S_{R C \ell} \triangle V\right), \quad \text { (9f } f\right) \\
& \left(S_{R C \ell}\right)_{p}=\gamma_{R m}\left(T_{R m}^{4}+3\left(T_{\ell}^{o}\right)^{4}\right)-4 \gamma_{R m}\left(T_{\ell}^{o}\right)^{3} T_{\ell},
\end{aligned}
$$

In equations (9a-f), $\ell$ denotes the phase index ( $g$ or $s)$. The subscripts $E, W, N$ and $S$ denote the cell centers of the East, West, North and South neighbor cells, respectively. The subscripts $e, w, n$ and $s$ denote the East, West, North and South face of the cell, respectively. The diffusion terms, $D_{f}$, where $f$ is the face of the cell, are modeled as Syamla, 1998, p. 19, Eq. 3.23)

$$
D_{f}=\frac{K_{\ell f} A_{f}}{\triangle x_{f}} .
$$

The $K_{\ell f}$ flux terms are given in (Syamla, 1998, p. 17, Eq. $3.6 \& 3.7$ ). $\xi, \epsilon, \rho$, and $C_{p}$ are the convection weighting factor, gas void fraction, density and constant volume specific heat, respectively. $u_{\ell}$ and $v_{\ell}$ are the phase velocities in the $x$ - and $y$-direction. $A$ is the area of 
a cell face. The superscript $o$ denotes "old" variables, that is, variables from the previous time step. The infinitesimal cell volume is represented by $\triangle V$. The radiation source term, $S_{R C \ell}$, is not modeled in the ODEt code.

The projection of the scalar energy equation onto the temperature basis functions is derived below. First, the scalar energy equation for temperature can be written as

$$
a_{P}\left(T_{\ell}\right)_{P}=\sum_{n b} a_{n b}\left(T_{\ell}\right)_{n b}+b_{P}
$$

where $\ell$ denotes either the gas or solids phase ( $g$ or $s$ ). Next, equations (9)e and (9) $f$ are substituted into Equation (10). The source terms present in the new expression are combined into the term $S_{\ell}^{T}$ for convenience.

$$
-\sum_{n b}\left(a_{m}^{T}\right)_{n b}\left(T_{\ell}\right)_{P}-\frac{\rho_{\ell} C_{p_{\ell}} \Delta V}{\triangle t}\left(T_{\ell}\right)_{P}=-\sum_{n b} a_{n b}\left(T_{\ell}\right)_{n b}-\frac{\rho_{\ell} C_{p_{\ell}} \Delta V}{\triangle t}\left(T_{\ell}^{o}\right)_{P}+S_{\ell}^{T}
$$

Equation (11) can be rearranged as

$$
-\rho_{\ell} C_{p_{\ell}} \Delta V \frac{\left(T_{\ell}\right)_{p}-\left(T_{\ell}^{o}\right)_{p}}{\Delta t}=\sum_{n b} a_{n b}\left(\left(T_{\ell}\right)_{n b}-\left(T_{\ell}\right)_{p}\right)+S_{\ell}^{T}
$$

Replacing $\frac{\left(T_{\ell}\right)_{p}-\left(T_{\ell}^{o}\right)_{p}}{\Delta t}$ with $\frac{\partial T_{\ell}}{\partial t}$ yields

$$
-\rho_{\ell} C_{p_{\ell}} \Delta V \frac{\partial T_{\ell}}{\partial t}=\sum_{n b} a_{n b}\left(\left(T_{\ell}\right)_{n b}-\left(T_{\ell}\right)_{p}\right)+S_{\ell}^{T}
$$

The temperature $T_{\ell}$ is approximated using the POD basis functions and time coefficients in the following equation:

$$
T_{\ell}(x, t) \cong \phi_{0}^{T_{\ell}}(x)+\sum_{i=1}^{m^{T_{\ell}}} \alpha_{i}^{T_{\ell}}(t) \phi_{i}^{T_{\ell}}(x)
$$

Substituting Equation (13) into Equation (12) and dropping the $x$ and $t$ variables yields

$$
-\rho_{\ell} C_{p_{\ell}} \Delta V \sum_{i=1}^{m^{T_{\ell}}} \dot{\alpha}_{i}^{T_{\ell}} \phi_{i}^{T_{\ell}}=\sum_{n b} a_{n b} \sum_{i=1}^{m^{T_{\ell}}}\left(\phi_{i, n b}^{T_{\ell}}-\phi_{i}^{T_{\ell}}\right) \alpha_{i}^{T_{\ell}}+S_{\ell}^{T}
$$


Substituting the $a$ coefficients given in equations (9)a - (9)d yields

$$
\begin{aligned}
\rho \Delta V \cdot \sum_{i=1}^{m_{\ell}^{T_{\ell}}} \dot{\alpha}_{i}^{T_{\ell}} \phi_{i}^{T_{\ell}}= & -\sum_{i=0}^{m^{T_{\ell}}} \sum_{j=0}^{m^{u}} \frac{\left(\xi_{\ell}^{T}\right)_{e}}{2}\left(\epsilon_{\ell} \rho_{\ell}\right)_{E}\left(C_{p_{\ell}}+C_{p_{\ell E}}\right) \Delta y \phi_{j, e}^{u_{\ell}}\left(\phi_{i, e}^{T_{\ell}}-\phi_{i}^{T_{\ell}}\right) \alpha_{i}^{T_{\ell}} \alpha_{j}^{u_{\ell}}+ \\
& +\sum_{i=0}^{m^{T_{\ell}}} \sum_{j=0}^{m^{u}} \frac{\left(\bar{\xi}_{\ell}^{T}\right)_{w}}{2}\left(\epsilon_{\ell} \rho_{\ell}\right)_{W}\left(C_{p_{\ell}}+C_{p_{\ell E}}\right) \Delta y \phi_{j, w}^{u_{\ell}}\left(\phi_{i, w}^{T_{\ell}}-\phi_{i}^{T_{\ell}}\right) \alpha_{i}^{T_{\ell}} \alpha_{j}^{u_{\ell}}- \\
& -\sum_{i=0}^{m_{\ell}^{T_{\ell}}} \sum_{j=0}^{m^{u}} \frac{\left(\xi_{\ell}^{T}\right)_{n}}{2}\left(\epsilon_{\ell} \rho_{\ell}\right)_{N}\left(C_{p_{\ell}}+C_{p_{\ell N}}\right) \Delta x \phi_{j, n}^{v_{\ell}}\left(\phi_{i, n}^{T_{\ell}}-\phi_{i}^{T_{\ell}}\right) \alpha_{i}^{T_{\ell}} \alpha_{j}^{v_{\ell}}+ \\
& +\sum_{i=0}^{m^{T} \ell} \sum_{j=0}^{m^{v}} \frac{\left(\bar{\xi}_{\ell}^{T}\right)_{s}}{2}\left(\epsilon_{\ell} \rho_{\ell}\right)_{S}\left(C_{p_{\ell}}+C_{p_{\ell N}}\right) \Delta x \phi_{j, s}^{v_{\ell}}\left(\phi_{i, s}^{T_{\ell}}-\phi_{i}^{T_{\ell}}\right) \alpha_{i}^{T_{\ell}} \alpha_{j}^{v_{\ell}}+ \\
& +\sum_{i=0}^{m^{T_{\ell}}}\left(K_{\ell}\right)_{e} \Delta y\left(\phi_{i, e}^{T_{\ell}}-\phi_{i}^{T_{\ell}}\right) \alpha_{i}^{T_{\ell}}+\sum_{i=0}^{m^{T_{\ell}}}\left(K_{\ell}\right)_{w} \Delta y\left(\phi_{i, w}^{T_{\ell}}-\phi_{i}^{T_{\ell}}\right) \alpha_{i}^{T_{\ell}}+ \\
& +\sum_{i=0}^{m^{T_{\ell}}}\left(K_{\ell}\right)_{n} \Delta x\left(\phi_{i, n}^{T_{\ell}}-\phi_{i}^{T_{\ell}}\right) \alpha_{i}^{T_{\ell}}+\sum_{i=0}^{m^{T_{\ell}}}\left(K_{\ell}\right)_{s} \Delta x\left(\phi_{i, s}^{T_{\ell}}-\phi_{i}^{T_{\ell}}\right) \alpha_{i}^{T_{\ell}}+ \\
& +S_{\ell}^{T}
\end{aligned}
$$

For two-dimensional flows, $A_{E}=A_{W}=\Delta y$ and $A_{N}=A_{S}=\Delta x$. Equation (14) is the finite volume discretized energy equation approximated using proper orthogonal decomposition. The $S_{\ell}^{T}$ terms are computed based on the previous time step values of the gas and solids temperatures. Next, Equation (14) is projected onto the basis functions $\phi_{k}^{T_{\ell}}$, where $k \in$ $\left[1, m^{T_{\ell}}\right]$, and yields 


$$
\begin{aligned}
\rho \Delta V \quad \cdot & \sum_{i=1}^{m^{T_{\ell}}} \dot{\alpha}_{i}^{T_{\ell}}\left(\phi_{i}^{T_{\ell}}, \phi_{k}^{T_{\ell}}\right)= \\
& -\sum_{i=0}^{m^{T_{\ell}}} \sum_{j=0}^{m^{u}} \frac{\left(\xi_{\ell}^{T}\right)_{e}}{2}\left(\epsilon_{\ell} \rho_{\ell}\right)_{E}\left(C_{p_{\ell}}+C_{p_{\ell E}}\right) \Delta y \phi_{j, e}^{u_{\ell}}\left(\phi_{i, e}^{T_{\ell}}-\phi_{i}^{T_{\ell}}\right), \phi_{k}^{T_{\ell}} \alpha_{i}^{T_{\ell}} \alpha_{j}^{u_{\ell}}+ \\
& +\sum_{i=0}^{m^{T_{\ell}}} \sum_{j=0}^{m^{u}} \frac{\left(\bar{\xi}_{\ell}^{T}\right)_{w}}{2}\left(\epsilon_{\ell} \rho_{\ell}\right)_{W}\left(C_{p_{\ell}}+C_{p_{\ell E}}\right) \Delta y \phi_{j, w}^{u_{\ell}}\left(\phi_{i, w}^{T_{\ell}}-\phi_{i}^{T_{\ell}}\right), \phi_{k}^{T_{\ell}} \alpha_{i}^{T_{\ell}} \alpha_{j}^{u_{\ell}}- \\
& -\sum_{i=0}^{m^{T_{\ell}}} \sum_{j=0}^{m^{u}} \frac{\left(\xi_{\ell}^{T}\right)_{n}}{2}\left(\epsilon_{\ell} \rho_{\ell}\right)_{N}\left(C_{p_{\ell}}+C_{p_{\ell N}}\right) \Delta x \phi_{j, n}^{v_{\ell}}\left(\phi_{i, n}^{T_{\ell}}-\phi_{i}^{T_{\ell}}\right), \phi_{k}^{T_{\ell}} \alpha_{i}^{T_{\ell}} \alpha_{j}^{v_{\ell}}+ \\
& +\sum_{i=0}^{m^{T_{\ell}}} \sum_{j=0}^{m^{v}} \frac{\left(\bar{\xi}_{\ell}^{T}\right)_{s}}{2}\left(\epsilon_{\ell} \rho_{\ell}\right)_{S}\left(C_{p_{\ell}}+C_{p_{\ell N}}\right) \Delta x \phi_{j, s}^{v_{\ell}}\left(\phi_{i, s}^{T_{\ell}}-\phi_{i}^{T_{\ell}}\right), \phi_{k}^{T_{\ell}} \alpha_{i}^{T_{\ell}} \alpha_{j}^{v_{\ell}}+ \\
& +\sum_{i=0}^{m^{T_{\ell}}}\left(K_{\ell}\right)_{e} \Delta y\left(\left(\phi_{i, e}^{T_{\ell}}-\phi_{i}^{T_{\ell}}\right), \phi_{k}^{T_{\ell}}\right) \alpha_{i}^{T_{\ell}}+\sum_{i=0}^{m^{T_{\ell}}}\left(K_{\ell}\right)_{w} \Delta y\left(\left(\phi_{i, w}^{T_{\ell}}-\phi_{i}^{T_{\ell}}\right), \phi_{k}^{T_{\ell}}\right) \alpha_{i}^{T_{\ell}}+ \\
& +\sum_{i=0}^{m^{T_{\ell}}}\left(K_{\ell}\right)_{n} \Delta x\left(\left(\phi_{i, n}^{T_{\ell}}-\phi_{i}^{T_{\ell}}\right), \phi_{k}^{T_{\ell}}\right) \alpha_{i}^{T_{\ell}}+\sum_{i=0}^{m^{T_{\ell}}}\left(K_{\ell}\right)_{s} \Delta x\left(\left(\phi_{i, s}^{T_{\ell}}-\phi_{i}^{T_{\ell}}\right), \phi_{k}^{T_{\ell}}\right) \alpha_{i}^{T_{\ell}}+ \\
& +\left(S_{\ell}^{T}, \phi_{k}^{T_{\ell}}\right) .
\end{aligned}
$$

Equation (15) can be written as

$$
\check{\mathcal{A}}_{k k}^{T_{\ell}} \dot{\alpha}_{k}^{T_{\ell}}=\sum_{i=0}^{m^{T_{\ell}}} \sum_{j=0}^{m^{u}} \check{\mathcal{F}}_{k i j}^{T_{\ell}} \alpha_{i}^{T_{\ell}} \alpha_{j}^{u_{\ell}}+\sum_{i=0}^{m^{T_{\ell}}} \sum_{j=0}^{m^{v}} \check{\mathcal{G}}_{k i j}^{T_{\ell}} \alpha_{i}^{T_{\ell}} \alpha_{j}^{v_{\ell}}+\sum_{i=0}^{m^{T_{\ell}}} \check{\mathcal{H}}_{k i}^{T_{\ell}} \alpha_{i}^{T_{\ell}}+\check{\mathcal{S}}_{\ell}^{T},
$$

where

$$
\begin{aligned}
\check{\mathcal{A}}_{i j}^{T_{\ell}}= & \delta_{i j} \cdot\left(\rho_{p} \phi_{j}^{T_{\ell}} \Delta V, \phi_{i}^{T_{\ell}}\right), \\
\check{\mathcal{F}}_{k i j}^{T_{\ell}}= & \left(-\left(\xi_{\ell}^{T_{\ell}} \epsilon_{\ell} \rho_{\ell} C_{p_{\ell}}\right)_{e} \Delta y \phi_{j, e}^{u_{\ell}}\left(\phi_{i, e}^{T_{\ell}}-\phi_{i}^{T_{\ell}}\right), \phi_{k}^{T_{\ell}}\right)+\left(\left(\bar{\xi}_{\ell}^{T_{\ell}} \epsilon_{\ell} \rho_{\ell}\right)_{w}\left(C_{p_{\ell}}\right)_{e} \Delta y \phi_{j, w}^{u_{\ell}}\left(\phi_{i, w}^{T_{\ell}}-\phi_{i}^{T_{\ell}}\right), \phi_{k}^{T_{\ell}}\right) \\
\check{\mathcal{G}}_{k i j}^{T_{\ell}}= & \left(-\left(\xi_{\ell}^{T_{\ell}} \epsilon_{\ell} \rho_{\ell} C_{p_{\ell}}\right)_{n} \Delta x \phi_{j, e}^{v_{\ell}}\left(\phi_{i, e}^{T_{\ell}}-\phi_{i}^{T_{\ell}}\right), \phi_{k}^{T_{\ell}}\right)+\left(\left(\bar{\xi}_{\ell}^{T_{\ell}} \epsilon_{\ell} \rho_{\ell}\right)_{s}\left(C_{p_{\ell}}\right)_{n} \Delta x \phi_{j, w}^{v_{\ell}}\left(\phi_{i, w}^{T_{\ell}}-\phi_{i}^{T_{\ell}}\right), \phi_{k}^{T_{\ell}}\right) \\
\check{\mathcal{H}}_{l i}^{T_{\ell}}= & \frac{\left(K_{\ell}\right)_{e} \Delta y}{\Delta x}\left(\phi_{i, e}^{T_{\ell}}-\phi_{i}^{T_{\ell}}\right), \phi_{k}^{T_{\ell}}+\frac{\left(K_{\ell}\right)_{w} \Delta y}{\Delta x}\left(\phi_{i, w}^{T_{\ell}}-\phi_{i}^{T_{\ell}}\right), \phi_{k}^{T_{\ell}} \\
& \quad+\frac{\left(K_{\ell}\right)_{n} \Delta x}{\Delta y}\left(\phi_{i, n}^{T_{\ell}}-\phi_{i}^{T_{\ell}}\right), \phi_{k}^{T_{\ell}}+\frac{\left(K_{\ell}\right)_{s} \Delta x}{\Delta y}\left(\phi_{i, s}^{T_{\ell}}-\phi_{i}^{T_{\ell}}\right), \phi_{k}^{T_{\ell}}, \\
\check{\mathcal{S}}_{k}^{T_{\ell}}= & \left(S^{T_{\ell}}, \phi_{k}^{T_{\ell}}\right) .
\end{aligned}
$$


In Equation (16) the original energy balance equations have been reduced to a set of $m^{T_{\ell}}$ ordinary differential equations. Equation (16) can be rewritten in a simpler form in terms of coefficients $a_{p}^{T_{\ell}}, a_{n b}^{T_{\ell}}, b_{p}^{T_{\ell}}$ and unknowns, $\alpha^{T_{\ell}}$.

$$
\tilde{\mathcal{A}}^{T_{\ell}} \alpha^{T_{\ell}}=\tilde{\mathcal{B}}^{T_{\ell}}
$$

where

$$
\begin{aligned}
& \tilde{\mathcal{A}}_{i j}^{T_{\ell}}=\left(\left(a_{p}^{T_{\ell}} \phi_{j}^{T_{\ell}}-\sum_{n b} a_{n b}^{T_{\ell}} \phi_{j, n b}^{T_{\ell}}\right), \phi_{i}^{T_{\ell}}\right), \\
& \tilde{\mathcal{B}}_{i}^{T_{\ell}}=\left(b_{p}^{T_{\ell}}, \phi_{i}^{T_{\ell}}\right) .
\end{aligned}
$$

Herein the dimensions of $\tilde{\mathcal{A}}^{T_{\ell}}$ and $\tilde{\mathcal{B}}^{T_{\ell}}$ are $m^{T_{\ell}} \times m^{T_{\ell}}$ and $m^{T_{\ell}} \times 1$, respectively.

\subsubsection{Implementation of the Energy Balance Equations in ODEt}

The reduced-order model of the energy equations has been implemented in ODEt. The ODEt code uses an iterative, time marching algorithm which is similar to the algorithm used by MFIX. An outline of the iterative loop of the ODEt solution algorithm is given below:

- Using the time coefficients from the previous iteration or those read in from the initial condition file for the first time step, the field variables $u_{g}, v_{g}, u_{s}, v_{s}, p_{g}, \epsilon_{g}, T_{g}$ and $T_{s}$ are reconstructed. For compressible flows, the density $\rho$ is calculated using the ideal gas law with the previous or reconstructed gas temperature. The physical properties $\rho_{g}$ and $\rho_{s}$ are calculated. The transport properties $\mu_{g}, \mu_{s}$ and $F_{g s}$ are calculated. The temperature dependent conductivity, $K_{m}$, and heat transfer coefficient, $\gamma_{g s}$, are calculated using the previous temperature field values.

- The linear systems of equations (Yuan et al, 2005, p. 248, Eq. 30 \& 31) are solved to obtain the tentative values of $\alpha_{i}^{u_{g}}(t), i \in\left[1, m^{u_{g}}\right], \alpha_{i}^{v_{g}}(t), i \in\left[1, m^{v_{g}}\right], \alpha_{i}^{u_{s}}(t), i \in\left[1, m^{u_{s}}\right]$ and $\alpha_{i}^{v_{s}}(t), i \in\left[1, m^{v_{s}}\right]$ where $m$ indicates the number of modes used. The values are called tentative because they are calculated based on the previous pressure field and will be corrected by the gas pressure correction and the solids volume fraction correction respectively.

- The linear system of equations (Yuan et a1. 2005, p. 249, Eq. 32) is solved to obtain $\alpha_{i}^{p_{g}^{\prime}}(t), i \in\left[1, m^{p_{g}}\right]$, the time coefficients of the gas pressure correction.

- The time coefficients of $p_{g}, u_{g}$ and $v_{g}$ are corrected using the new value of $\alpha_{i}^{p_{g}^{\prime}}(t)$.

- The linear system of equations (6) is solved to obtain $\alpha_{i}^{\epsilon_{s}{ }^{\prime}}(t), i \in\left[1, m^{\epsilon_{s}}\right]$, the time coefficients of the solids volume fraction correction. The void fraction, $\epsilon_{g}$ is calculated using $\epsilon_{g}=1-\epsilon_{s}$. 
- The time coefficients of $\epsilon_{s}, u_{s}$ and $v_{s}$ are corrected using the new $\alpha_{i}^{\epsilon_{s}{ }^{\prime}}(t)$ values.

- The linear systems of equations (17) are solved to obtain $\left(\alpha_{i}^{T_{g}}\right)(t), i \in\left[1, m^{T_{g}}\right]$ and $\alpha_{i}^{T_{s}}(t), i \in\left[1, m^{T_{s}}\right]$, the time coefficients of the gas and solids phase temperature.

- Check the convergence. If the solution is converged, ODEt advances to the next time step.

The input data for ODEt are the basis functions extracted from the database of eight field variables generated using MFIX. The eight field variables are $x$ - and $y$-direction gas and solids velocities, gas pressure, void fraction, and gas and solids temperatures. The solutions of ODEt are $\alpha_{i}^{u_{g}}, i \in\left[1, m^{u_{g}}\right], \alpha_{i}^{v_{g}}, i \in\left[1, m^{v_{g}}\right], \alpha_{i}^{u_{s}}, i \in\left[1, m^{u_{s}}\right], \alpha_{i}^{v_{s}}, i \in\left[1, m^{v_{s}}\right],\left(\alpha_{i}^{p_{g}}\right)^{\prime}$, $i \in\left[1, m^{p_{g}}\right],\left(\alpha_{i}^{\epsilon_{g}}\right)^{\prime}, i \in\left[1, m^{\epsilon_{g}}\right], \alpha_{i}^{T_{g}}(t), i \in\left[1, m^{T_{g}}\right]$ and $\alpha_{i}^{T_{s}}(t), i \in\left[1, m^{T_{s}}\right]$.

Table 0 shows a list of the most important subroutines created for the POD-based reduced-order model, ODE3x. The routines in Table 0 are especially important because they are used to perform the projection of the energy balance equations onto the temperature basis functions, solve the linear system of equations and reconstruct the temperature values from the computed time coefficients, $\alpha_{i}^{T_{\ell}}$.

Table 7: Most important routines created for ODEt.

\begin{tabular}{l|l}
\hline Subroutine & \multicolumn{1}{c}{ Description } \\
\hline calc_ab_phi.f & $\begin{array}{l}\text { Projects the energy equation system onto the basis } \\
\text { functions extracted from the temperature snapshots } \\
\text { Calculates the constant basis function products } \\
\text { phi_phi_prod.f }\end{array}$ \\
reconstruct_t_m.f & $\begin{array}{l}\text { Reconstructs the gas and solids phase temperatures } \\
\text { from the basis functions and time coefficients } \\
\text { Replaces original leq_bicgs with LU decomposition solver }\end{array}$ \\
\hline
\end{tabular}

Table 8 shows a list of the most important subroutines modified for ODEt. In general, the modified routines retain the same names used in MFIX. Most of the modifications involved adding new terms for the POD algorithm.

Table 9 shows a list of support subroutines modified for ODEt. The routines in Table 9 are called support routines because they are not directly used for calculation. These subroutines are used for data input/output and for declaring new variables.

\subsubsection{Test, Verify and Validate ODEt}

In order to verify and validate ODEt against MFIX, a non-isothermal test case was used. The boundary conditions for this case are shown in Figure 1 and the specific parameters are given in Table 6. The values used for the initial specific heat coefficients $C_{p g 0}$ and $C_{p s 0}$ 
Table 8: Most important routines modified for ODEt.

\begin{tabular}{|c|c|}
\hline Subroutine & Modification \\
\hline allocate_arrays.f & $\begin{array}{l}\text { Defined dynamic allocation size for the basis } \\
\text { function arrays, time coefficients, constant basis } \\
\text { function products and all new energy equation } \\
\text { parameters not defined in ODEx. }\end{array}$ \\
\hline calc_mu_g.f & Implemented temperature dependent $\mu_{g}$ calculation \\
\hline calc $_{-}$ & Implemented 2-D, 2-phase version of routines \\
\hline calc_gamma & Implemented 2-D, 2-phase version of routine \\
\hline conv_dif_phi.f & Implemented 2-D, 2-phase version of routine \\
\hline bc_p & Implemented no slip wall condition \\
\hline iters & Added write out operations for $\alpha^{T_{\ell}}$ \\
\hline cal_prop.f & Sets $C_{p_{\ell}}, \gamma_{R \ell}$, and $\Delta H_{r \ell}$ from input data \\
\hline sour & Implemented 2-D, 2-phase version of routine \\
\hline solve & $\begin{array}{l}\text { Modified the call line for the linear equation } \\
\text { solver and calc_resid_s to solve for the time } \\
\text { coefficient of temperature, } \alpha^{T_{\ell}} \text {. Added the } \\
\text { call line for the new calc_ab_phi routine. Added } \\
\text { error flag to identify if } T<0 \text {. }\end{array}$ \\
\hline time_march.f & $\begin{array}{l}\text { Added lines to open and write out computed time } \\
\text { coefficients to the output files. }\end{array}$ \\
\hline & Added call statements for reconstruct_t routines. \\
\hline
\end{tabular}


Table 9: Support routines modified for ODEt.

\begin{tabular}{|c|c|}
\hline Subroutine & Modification \\
\hline $\begin{array}{l}\text { basis_mod.f } \\
\text { get_data.f }\end{array}$ & $\begin{array}{l}\text { Added temperature basis function definitions } \\
\text { Added statement to print error flag for undefined input }\end{array}$ \\
\hline namelist.inc & $\begin{array}{l}\text { Added number of modes for temperature parameters, } \mathrm{n}_{-} T \mathrm{Tm} \text {, } \\
\text { and initial values of } C_{p_{\ell}}, \gamma_{R \ell} \text {, and } \Delta H_{r \ell} \text { to list of } \\
\text { parameters to be read from the input file, puv.dat }\end{array}$ \\
\hline param_mod.f & $\begin{array}{l}\text { Added definitions for } \mathrm{nT}_{-} \mathrm{g} \text { and } \mathrm{nT}_{-} \mathrm{s} \text {, the integer } \\
\text { number of modes used for temperature reconstruction }\end{array}$ \\
\hline read_basis.f & $\begin{array}{l}\text { Modified routine to read the temperature basis functions } \\
\text { from the PODDEC generated basis function files }\end{array}$ \\
\hline set_ic.f & $\begin{array}{l}\text { Modified routine to read the first time coefficients for } \\
\text { temperature from the PODDEC generated time coefficient } \\
\text { files to generate an initial field solution }\end{array}$ \\
\hline time_coe_mod.f & $\begin{array}{l}\text { Added definitions for } \alpha_{g}^{T} \text { and } \alpha_{s}^{T} \text {, the gas and solids } \\
\text { phase temperature time coefficients }\end{array}$ \\
\hline
\end{tabular}

are those for air and ash at a temperature of $297 \mathrm{~K}$, respectively. In this case, radiation and internal heat sources are not modeled. Heat transfer is achieved only by convection and diffusion between hot gas entering the control volume and the gas and solids bed at an initially cooler temperature. The particle diameter is assumed to be uniform and constant. Both the gas and solids phases are modeled as a single species and the flow is assumed to be non-reacting.

Figure 9 compares the gas and solids temperature fields computed using MFIX and ODEt at $t=1 \mathrm{~s}$ and Figure 10 shows the same fields in greater detail around the jet inlet. The agreement is very good. This agreement is also evident in the time coefficients, shown in Figures 11 and 12 . 


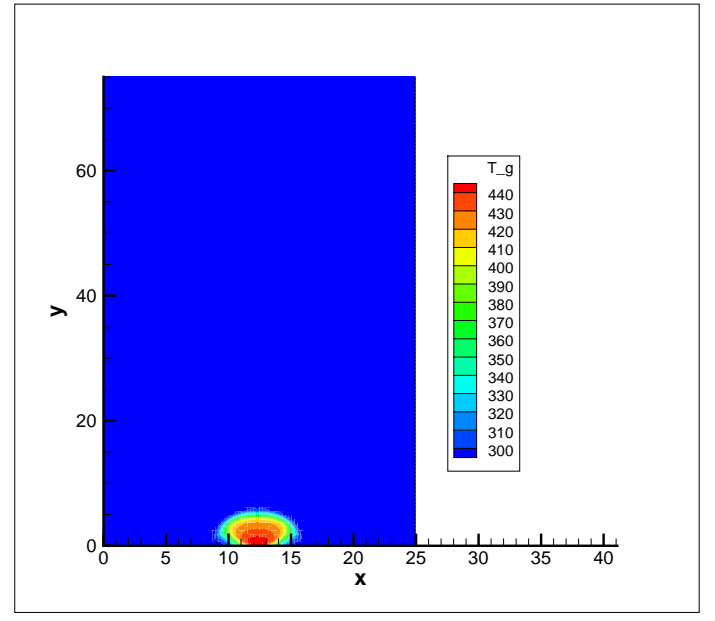

(a)

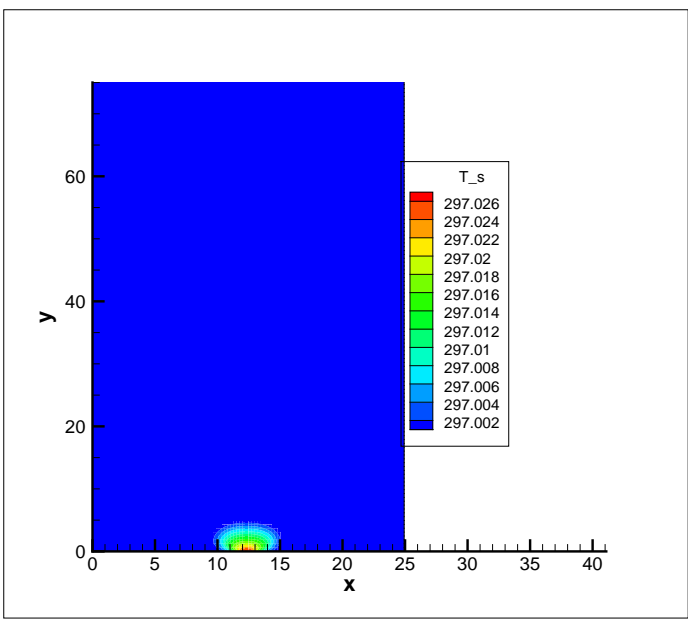

(c)

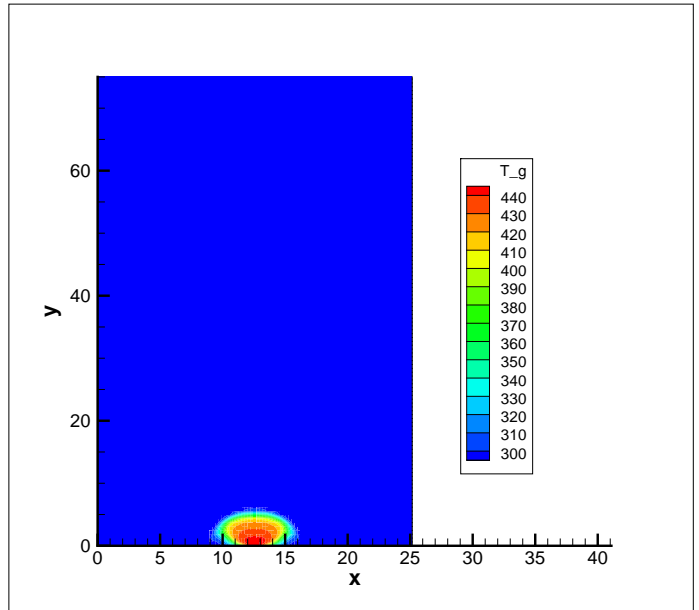

(b)

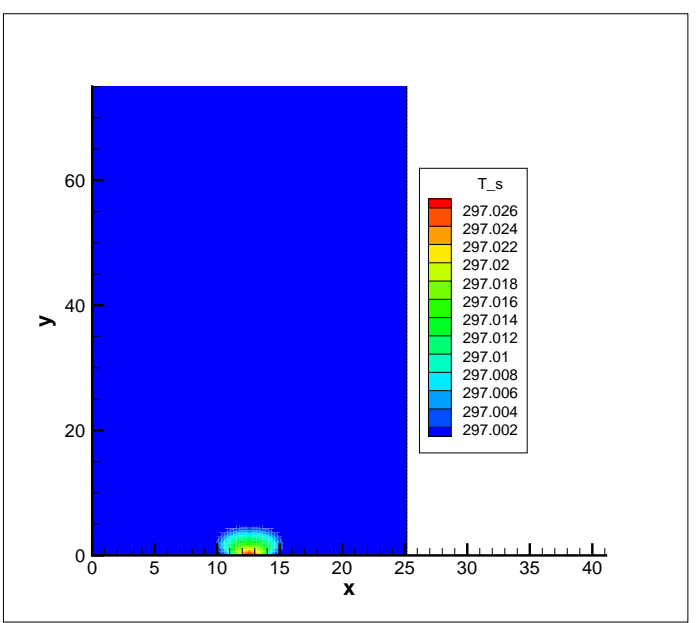

(d)

Figure 9: Contour plots at $t=1.0 \mathrm{~s}$ for: (a) gas temperature, MFIX, (b) gas temperature, ODEt, (c) solids temperature, MFIX, and (d) solids temperature, ODEt. 


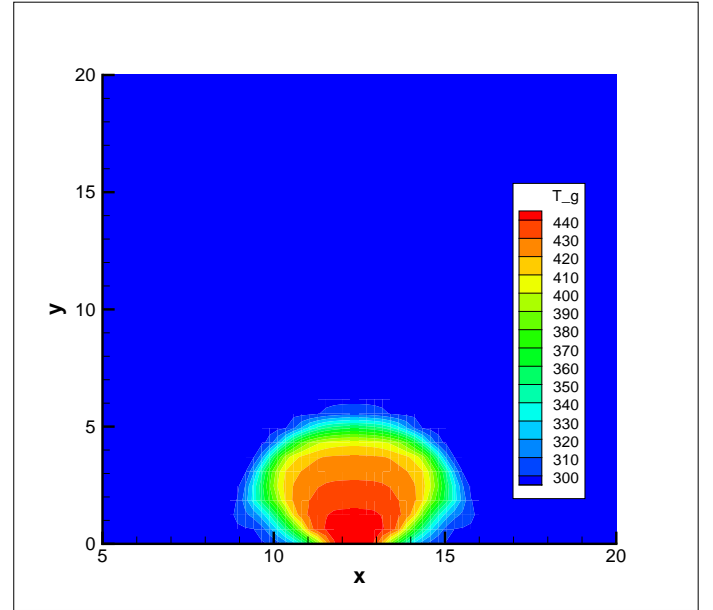

(a)

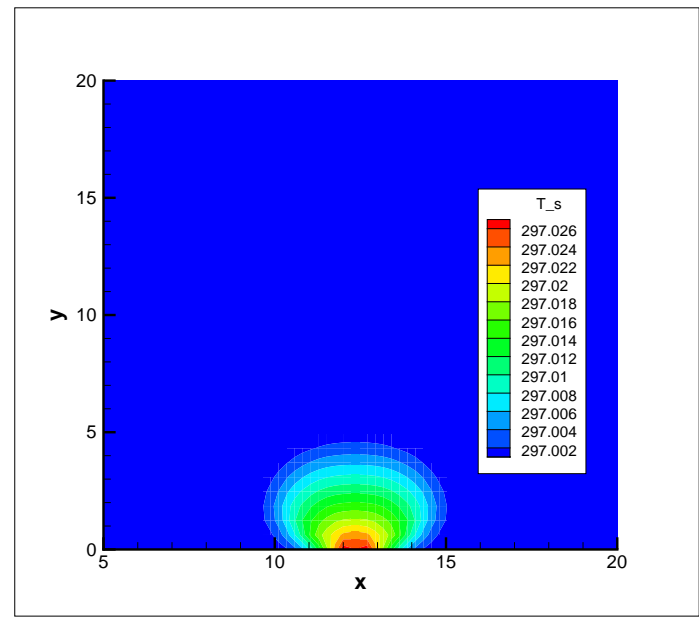

(c)

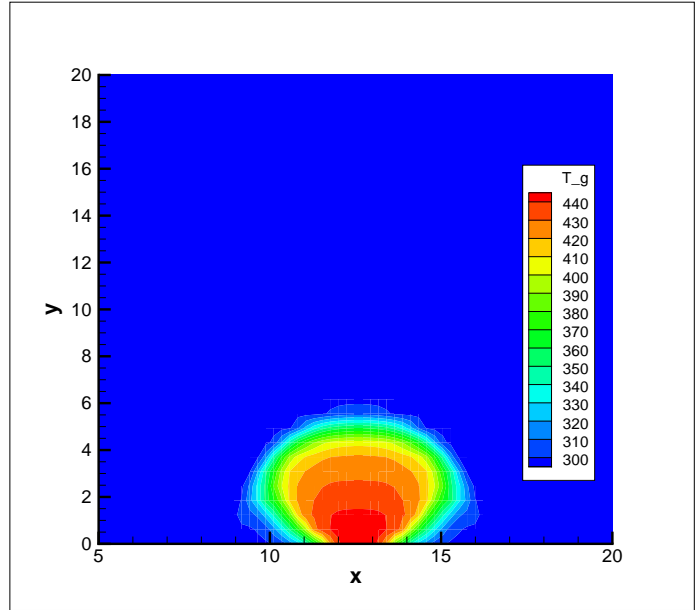

(b)

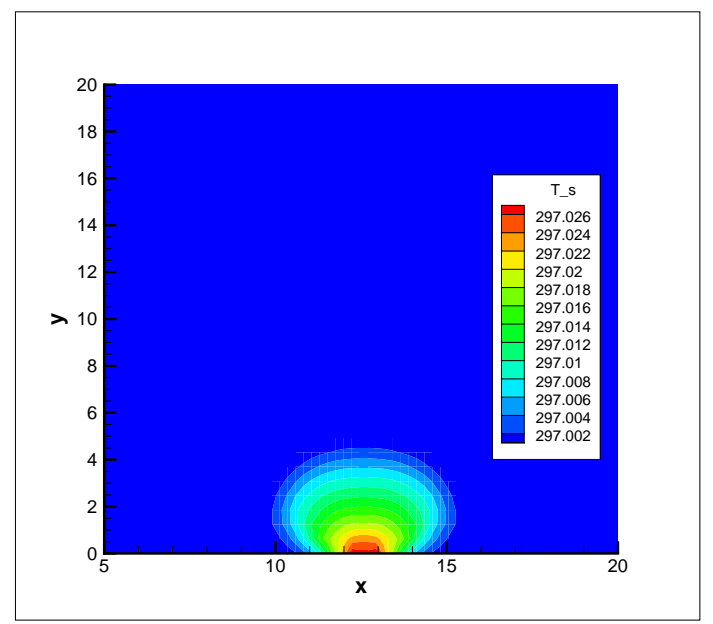

(d)

Figure 10: Contour plots showing the region around the jet inlet at $t=1.0 \mathrm{~s}$ for: (a) gas temperature, MFIX, (b) gas temperature, ODEt, (c) solids temperature, MFIX, and (d) solids temperature, ODEt. 


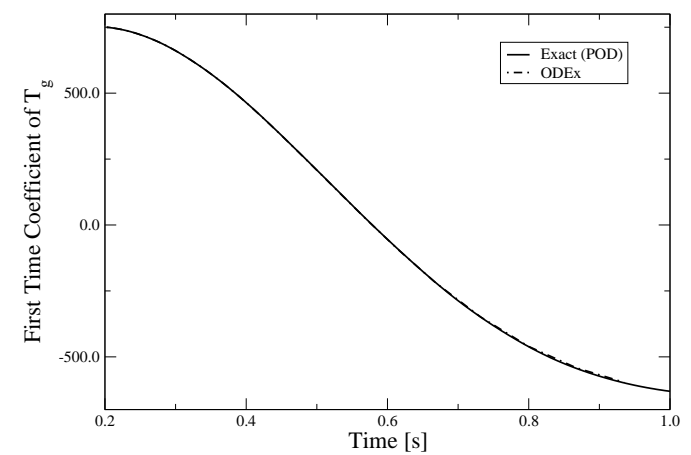

(a)

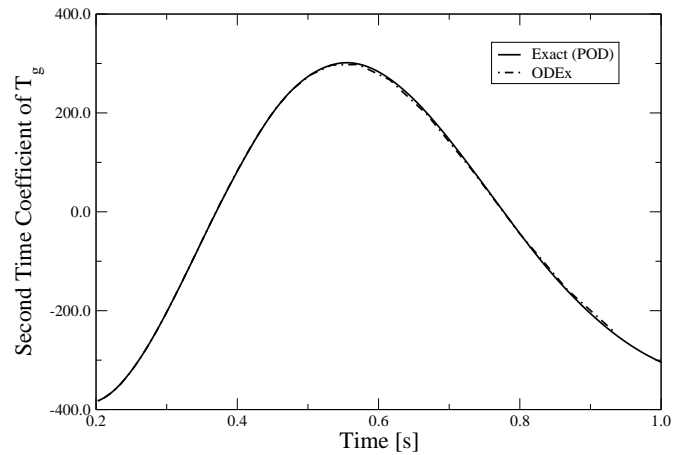

(b)

Figure 11: Comparison of time coefficients for solids temperature: (a) $\alpha_{1}^{T_{g}}$, and (b) $\alpha_{2}^{T_{g}}$.

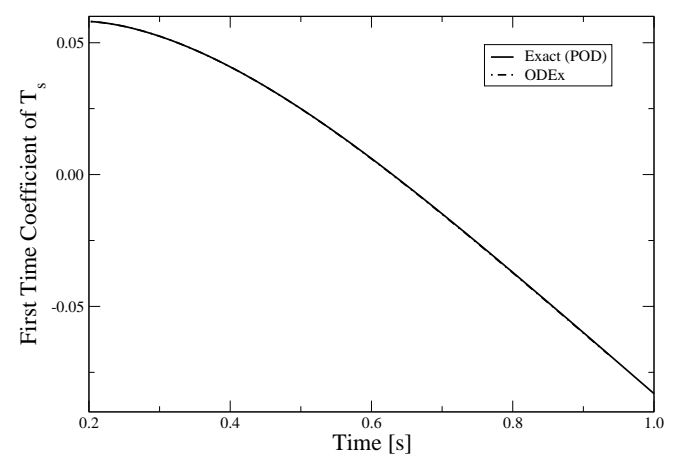

(a)

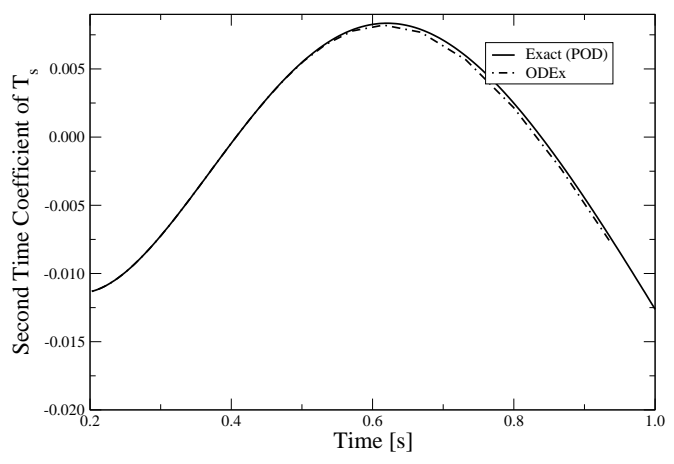

(b)

Figure 12: Comparison of time coefficients for solids temperature: (a) $\alpha_{1}^{T_{s}}$, and (b) $\alpha_{2}^{T_{s}}$. 


\subsubsection{Acceleration Methods for the ROM}

Several acceleration methods were explored as a means to speed up ODEx. An evaluation of the time efficiency and error for these methods is presented. The techniques presented herein are: (i) an algorithm for splitting the database, (ii) an algorithm for solving quasisymmetrical matrices, and (iii) a strategy for reducing the frequency of updating $\tilde{\mathcal{A}}$. The influence of a time step adjustment strategy on the time efficiency is also discussed. Finally, ODEx was used to extrapolate the field variables in time.

An algorithm for splitting the snapshot database was developed. The POD basis functions were extracted from a database of snapshots generated by numerically integrating the governing differential equations. Currently, it is common to use a database that includes all the snapshots. Using a single database that covers the entire time domain, however, could be too restrictive. For example, consider the transience during the start-up of the flow in a fluidized bed. The large variation in time at start-up requires more modes than are necessary to model the flow features present in the latter part of the simulation. A method to avoid this problem is to split the database of snapshots.

Splitting the database into multiple subsets produces an auto-correlation matrix $\overline{\bar{R}}$ that contains more relative energy in the first modes. $\overline{\bar{R}}$ is given by

$$
\overline{\bar{R}}(\mathbf{x}, \mathbf{y})=\sum_{i=1}^{M} \mathbf{u}\left(\mathbf{x}, t_{i}\right) \mathbf{u}^{T}\left(\mathbf{y}, t_{i}\right) / M
$$

Herein, energy is defined as the sum of all the POD eigenvalues. The relative energy captured by the $k$ th mode is defined as $\lambda_{k} / \sum_{i=1}^{M} \lambda_{i}$ (Cizmas and Palacios, 2003). As the relative energy of the first modes increases, fewer POD modes are needed in the reconstruction

$$
u\left(x, t_{i}\right)=\sum_{k=1}^{M} \alpha_{k}\left(t_{i}\right) \varphi_{k}(x), \quad i=1, \ldots, M
$$

to approximate the solution. Consequently, the computational cost of the reduced-order model decreases.

The snapshots created by solving the full-order model for the minimum fluidization case were divided into two parts. The first part, which captured the transient flow, ranged from 0.2 to $0.35 \mathrm{~s}$ and included 60 snapshots. The second part, which captured the slower varying flow, ranged from 0.35 to $1.0 \mathrm{~s}$ and included 260 snapshots. Figure 13 shows the cumulative energy of the POD modes obtained using a single database that covered the entire time domain. Figures 14 and 15 show the cumulative energy of the POD modes for the split databases.

The energy variation extracted from the 0.2-1.0 s database, shown in Fig. 13, was similar to the energy variation extracted from the transient snapshots, shown in Fig. 14. Most of the energy extracted from the 0.35-1.0 s database was, however, concentrated in the first mode, as shown in Fig. 15. This concentration of the energy allowed capturing most of the flow 


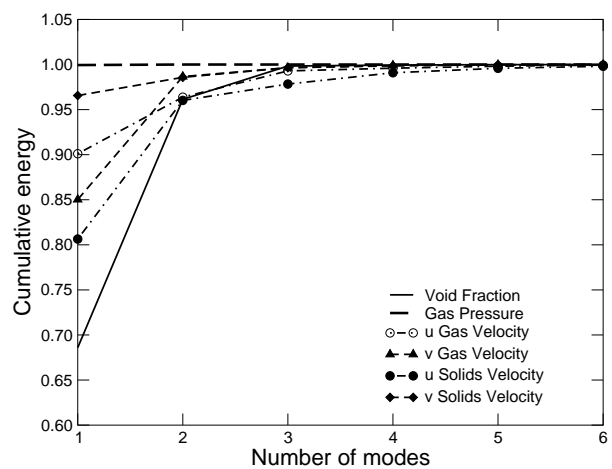

Figure 13: Cumulative energy spectrum for a database that spans 0.2-1.0 s.
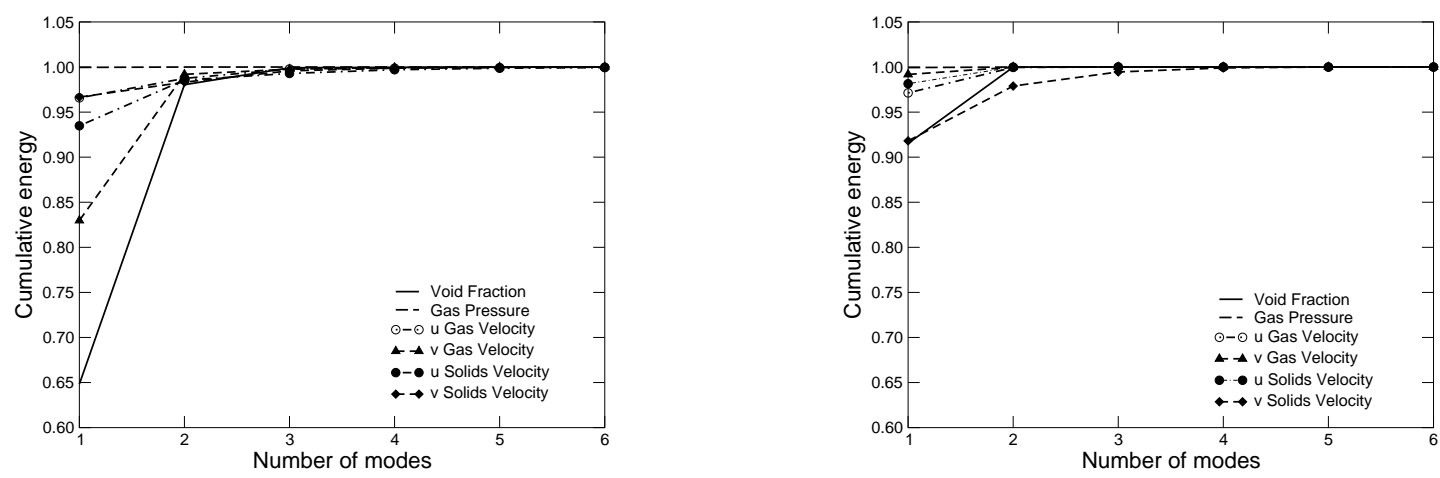

Figure 14: Cumulative energy spectrum for a Figure 15: Cumulative energy spectrum for a database that spans $0.2-0.35 \mathrm{~s}$. database that spans $0.35-1.0 \mathrm{~s}$. 
features using fewer modes compared to the transient regime. For the minimum fluidization case without database splitting, the reduced-order model with the modes given by Table 10 was 21 times faster then the full-order model. When the database was split at $t=0.35 \mathrm{~s}$, the number of modes used from 0.2 to $0.35 \mathrm{~s}$ was given by Table 10 and the number of modes used from 0.35 to $1.0 \mathrm{~s}$ was 1 for each variable. Splitting the database at $t=0.35 \mathrm{~s}$ resulted in a speed-up of 30 compared to the full-order model. The increase in the speed-up factor was the result of using fewer modes in the post transient period.

Table 10: Number of modes used and their symbols.

\begin{tabular}{lcc}
\hline Field variable & Symbol & Number of modes \\
\hline Gas pressure & $N_{p_{g}}$ & 2 \\
Solids volume fraction & $N_{\epsilon s}$ & 3 \\
$u$ gas velocity & $N_{u g}$ & 1 \\
$v$ gas velocity & $N_{v g}$ & 5 \\
$u$ solids velocity & $N_{u s}$ & 4 \\
$v$ solids velocity & $N_{v s}$ & 3 \\
\hline
\end{tabular}

The error of the reduced-order model with respect to the full-order model was defined as Yuan et al., 2005)

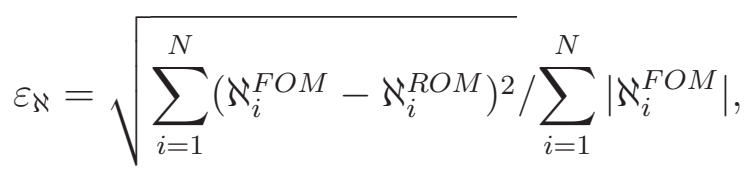

where $\aleph$ represents the dependent field variable, FOM denotes the full-order model, and $R O M$ denotes the reduced-order model with or without freezing. Here $N$ is the total number of spatial grid points. The errors of each variable were then used to calculate the average error as

$$
\varepsilon_{a v g}=\left(\varepsilon_{p_{g}}+\varepsilon_{\epsilon_{s}}+\varepsilon_{u_{g}}+\varepsilon_{v_{g}}+\varepsilon_{u_{s}}+\varepsilon_{v_{s}}\right) / 6
$$

In the case without database splitting the average error at $t=1.0 \mathrm{~s}$ was $1.882 \mathrm{E}-2$. The average error at $t=1.0 \mathrm{~s}$ while using database splitting was $4.370 \mathrm{E}-2$. The error increased in the latter case because slightly less energy was captured in the second interval.

The second acceleration method explored was a solver for linear systems with quasisymmetric matrices. Let us consider the system of equations generated by projecting the solids volume fraction correction equation onto the basis functions extracted from an ensemble of $\epsilon_{s}$ snapshots. This system can be written as

$$
\tilde{\mathcal{A}}^{\epsilon_{s}} \alpha^{\epsilon_{s}}=\tilde{\mathcal{B}}^{\epsilon_{s}},
$$


where $\alpha^{\epsilon_{s}}$ is the vector of unknowns $\alpha_{i}^{\epsilon_{s}}$. The $\ell k$ element of the $\tilde{\mathcal{A}}^{\epsilon_{s}}$ matrix is

$$
\tilde{\mathcal{A}}_{\ell k}^{\epsilon_{s}}=\left\{\varphi_{\ell}\right\}^{T}[A]\left\{\varphi_{k}\right\}-\sum_{n b=1}^{N B}\left\{\varphi_{\ell}\right\}^{T}\left[A_{n b}\right]\left\{\varphi_{k_{n b}}\right\}, \quad \ell, k=1, \ldots, m
$$

The $\tilde{\mathcal{A}}^{\epsilon_{s}}$ matrix is not symmetrical because of the second term $-\sum_{n b=1}^{N B}\left\{\varphi_{\ell}\right\}^{T}\left[A_{n b}\right]\left\{\varphi_{k_{n b}}\right\}$ of the element $\tilde{\mathcal{A}}_{\ell k}^{\epsilon_{s}}$. The matrix would be symmetrical if $\left\{\varphi_{k}\right\}=\left\{\varphi_{k_{n b}}\right\}$. The difference between the two vectors $\left\{\varphi_{k}\right\}$ and $\left\{\varphi_{k_{n b}}\right\}$ is small, however, because the latter vector is evaluated at slightly different spatial locations compared to the first vector. Similarly, the systems of linear algebraic equations for the other field variables have matrices that are not symmetrical. These matrices, however, are quite close to being symmetrical, and for this reason will be called quasi-symmetrical.

The algorithm proposed herein for solving a system of equations $A x=b$, in which the matrix $A$ must be positive definite and quasi-symmetrical, begins by splitting the matrix into a symmetrical and a non-symmetrical part (Cizmas et al., 2008):

$$
\left(A_{s}+A_{n}\right) x=b
$$

A solution of the symmetrical part is then obtained by solving the system of equations

$$
A_{s} x_{s}^{(1)}=b
$$

The solution of Equation (21) is decomposed in a component obtained by solving the system of equations (22) and a correction needed because the matrix $A$ is non-symmetrical

$$
x=x_{s}^{(1)}+x_{n}^{(1)}
$$

Substituting Equation (23) into Equation (21) and deducting Equation (22) yields

$$
\begin{aligned}
& \left(A_{s}+A_{n}\right) x_{n}^{(1)}=-A_{n} x_{s}^{(1)} \text { or } \\
& \left(A_{s}+A_{n}\right) x_{n}^{(1)}=b^{(1)}
\end{aligned}
$$

Note that the system of equations (24) has the same matrix as Equation (21). Consequently, an iterative process can be used to find the solution. $x_{n}^{(1)}$ can be considered a correction of the solution $x_{s}^{(1)}$ that is needed because the $A$ matrix is non-symmetrical. The correction $x_{n}^{(1)}$ can be split into two components: a component $x_{s}^{(2)}$ obtained by solving the system $A_{s} x_{s}^{(2)}=b^{(1)}$ and a correction needed because the matrix $A$ is non-symmetrical, similarly to the approach used in Equation (23):

$$
x_{n}^{(1)}=x_{s}^{(2)}+x_{n}^{(2)}
$$


Substituting Equation (25) into Equation (24) and using $A_{s} x_{s}^{(2)}=b^{(1)}$ yields

$$
\begin{aligned}
& \left(A_{s}+A_{n}\right) x_{n}^{(2)}=-A_{n} x_{s}^{(2)} \text { or } \\
& \left(A_{s}+A_{n}\right) x_{n}^{(2)}=b^{(2)}
\end{aligned}
$$

This process of approximating the solution yields after $p$ steps

$$
x=x_{s}^{(1)}+x_{s}^{(2)}+\cdots+x_{s}^{(p)}+x_{n}^{(p)},
$$

where the values $x_{s}^{(i)}, 1 \leq i \leq p$, are obtained by solving the linear system

$$
A_{s} x_{s}^{(i)}=b^{(i)}
$$

where $b^{(i)}=-A_{n} x_{s}^{(i-1)}$. The iterative process of adding corrections is stopped when $x_{s}^{(p)}$ is smaller than an imposed error.

The computation of $x_{s}^{(i)}$ requires the solution of the linear system of equations (28) several times. The Cholesky decomposition is used for the factorization of the positivedefinite symmetrical matrix $A_{s}$. The method takes advantage of the fact that the $A_{s}$ matrix is constant and only the right-hand-side vector $b^{(i)}$ changes.

The method proposed herein replaced the LU decomposition (Press et a1, 1992, p. 34) that was previously used to solve the linear algebraic systems for the field variables (Yuan et a1., 2005). For a system with $m$ equations, the number of operations for the LU decomposition is $m^{3} / 3$ while the number of operations for the Cholesky decomposition is $m^{3} / 6$. Numerical tests showed that three to four $x_{s}^{(i)}$ terms in Equation (27) were usually sufficient to obtain a solution with an error less than $10^{-6}$. Consequently, three to four $x_{s}^{(i)}$ solutions of the linear algebraic system of equations (28) must be computed. Since only the righthand-side term $b^{(i)}$ changes while the matrix $A_{s}$ is constant, the number of operations for the proposed method was increased by a factor proportional to $m^{2}$ multiplied by the number of $x_{s}^{(i)}$ terms in Equation (27). As long as $m$ is larger than 4, the computational cost of the proposed method is approximately half that of the LU decomposition. For additional details, see (Cizmas et al. 2008.

The third acceleration method that was explored was freezing the $\tilde{\mathcal{A}}$ matrix of the linear system. The projection of the discretized differential equation onto the basis functions takes most of the computational time of a subiteration. For a minimum fluidization case Yuan, $2003)$ numerical tests showed that the components of the projected $\tilde{\mathcal{A}}$ matrix do not vary significantly past the transient period. To quantify the variation of the $\tilde{\mathcal{A}}$ matrix, the eigenvalues of $\tilde{\mathcal{A}}(t)^{-1} \tilde{\mathcal{A}}(t+\Delta t)$, where $\Delta t$ is the subiteration time step, were compared against 1 , the eigenvalues of identity matrix. Table 11 shows the results of a comparison performed using the $\tilde{\mathcal{A}}^{v_{g}}$ matrices extracted from the transient and post transient periods. The eigenvalues varied by $4.1 \cdot 10^{-3} \%$ between subiterations in the transient period, and by $6.7 \cdot 10^{-7 \%}$ in the post transient period. Similar results were obtained for the other dependent variables. Since the $\tilde{\mathcal{A}}$ matrices of the linear systems did not vary significantly between two subitera- 
tions, they were updated every other iteration to reduce computational time. The $\tilde{\mathcal{B}}$ vectors, however, were updated every subiteration.

Table 11: Eigenvalues of $\tilde{\mathcal{A}}^{v^{-1}}(t) \tilde{\mathcal{A}}^{v_{g}}(t+\Delta t)$ during the transient $(t=0.25 \mathrm{~s})$ and post transient $(t=0.9 \mathrm{~s})$ regimes.

\begin{tabular}{cccccc}
\hline Time & \multicolumn{5}{c}{ Eigenvalues } \\
$t[\mathrm{~s}]$ & $\lambda_{1}$ & $\lambda_{2}$ & $\lambda_{3}$ & $\lambda_{4}$ & $\lambda_{5}$ \\
\hline 0.25 & 1.000000000 & 1.000000000 & 1.000000000 & 0.999982334 & 0.999936216 \\
0.90 & 1.000000000 & 1.000000000 & 1.000000000 & 1.000000000 & 0.999999933 \\
\hline
\end{tabular}

Due to the possibility of accumulating errors because of freezing the $\tilde{\mathcal{A}}$ matrix, skipping to update $\tilde{\mathcal{A}}$ was started only when the transient period ended. The relationship between the computational time and the physical time was used herein to determine when to stop updating the matrix every subiteration. Figure 16 shows that for $t>0.4 \mathrm{~s}$ the relationship between the CPU time and the physical time was close to linear. This indicates that the transient period ends at $t=0.4 \mathrm{~s}$.

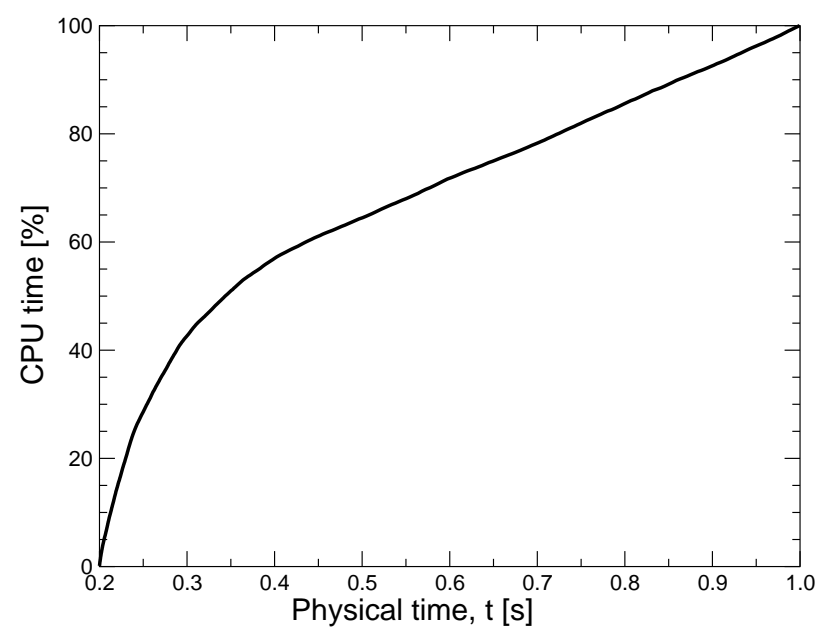

Figure 16: Relationship between physical time and CPU time.

The application of freezing resulted in a $10.1 \%$ decrease of computational time while using the modes from Table 10. There was, however, an increase of the error compared to the reduced-order model without freezing. The error of the reduced-order model with respect to the full-order model was defined by Equation (19). The error of the ROM with freezing, 
$\varepsilon^{F}$, with respect to the ROM without freezing, $\varepsilon^{N F}$, was defined as $\varepsilon^{F-N F}=\left|\varepsilon^{N F}-\varepsilon^{F}\right| / \varepsilon^{N F}$. Table 12 shows the errors of some of the dependent field variables at time $t=1 \mathrm{~s}$, the end of the integration period. The largest error $\varepsilon^{F-N F}$ corresponds to the solids volume fraction. The errors between the reduced-order model and the full-order model for the solids volume fraction are extremely small. Consequently, the increase of the error $\varepsilon_{\epsilon_{s}}^{F-N F}$ in this case is not a concern.

Table 12: Errors at $t=1.0 \mathrm{~s}$ with and without freezing using POD modes specified by Table 10 .

\begin{tabular}{clll}
\hline Variable & $\varepsilon^{N F}[\%]$ & $\varepsilon^{F}[\%]$ & $\varepsilon^{F-N F}[\%]$ \\
\hline$u_{g}$ & 0.215 & 0.224 & 0.419 \\
$v_{g}$ & 0.340 & 0.362 & 6.471 \\
$p_{g}$ & $1.544 \cdot 10^{-5}$ & $1.643 \cdot 10^{-5}$ & 6.412 \\
$\epsilon_{s}$ & $4.159 \cdot 10^{-5}$ & $5.059 \cdot 10^{-5}$ & 21.640 \\
\hline
\end{tabular}

Given that freezing the projection of the $\tilde{\mathcal{A}}$ matrix greatly reduces the number of operations per subiteration, a $10.1 \%$ decrease of CPU time is a small improvement. This limited reduction of the computational time was due to two factors. First, freezing was applied beginning at time $t=0.4 \mathrm{~s}$ and for this reason affected only a portion of the integration time. Second, an increase in the number of subiterations diminished the benefit of saving computational time by not updating the $\tilde{\mathcal{A}}$ matrix.

The effect of the initial time step on the computational time was also explored. The FOM and the ROM use an identical method to adjust the time step during the integration. This method can vary the size and the frequency of the time step adjustment. Given identical initial time steps and time step adjustment parameters, the time step size in the ROM increased by at least one order of magnitude, while the time step in the FOM remained almost constant, as shown in Fig. 17.

Three parameters were modified in the ROM to reduce the computation time: (1) the frequency of the time step adjustment, (2) the size of the time step adjustment, and (3) the size of the initial time step. Numerical tests showed that the variation of the initial time step size was the most effective time step adjustment speed-up method. The initial time step for the minimum fluidization case was varied between $10^{-4}$ and $10^{-3} \mathrm{~s}$. Increasing the initial time step size from $10^{-4}$ to $10^{-3}$ s reduced the computational time between 6.1 and $22.3 \%$, depending on the number of modes used for the minimum fluidization case. This relatively small reduction in computational time did not reflect the increase of the initial time step. Much of the computational speed-up was lost because the size of the time step was reduced during the integration in the transient period to satisfy convergence.

The optimal value for reducing the computational time was found to be $5 \cdot 10^{-4} \mathrm{~s}$ while the time step was adjusted by $\pm 10 \%$ every 5 iterations. Increasing the initial time step above the optimal value did not diminish the computational time because the time step must be 


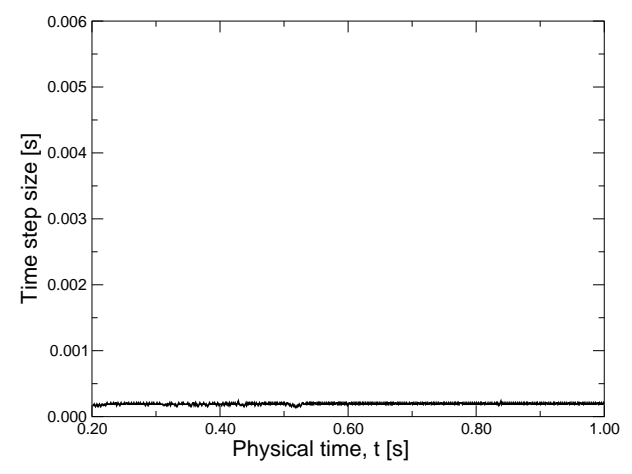

(a)

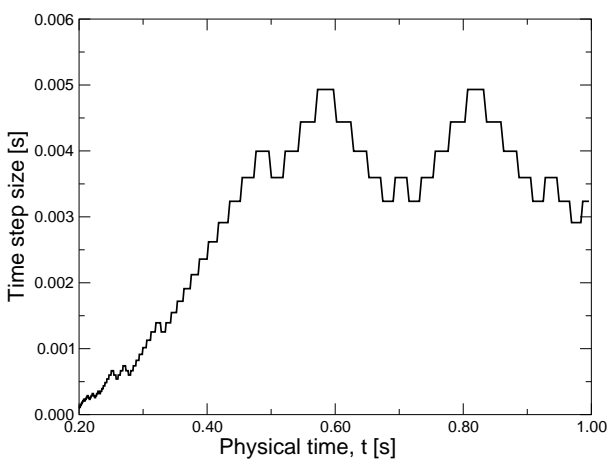

(b)

Figure 17: Time step size vs. physical time: (a) FOM, and (b) ROM.

reduced to achieve convergence. On the other hand, when the initial time step was smaller than the optimal value, the time steps did not grow as large as in the case with optimal initial time step.

A summary of the speed-up factors achieved by the various versions of the reduced-order model is given in Table 13. The speed-up factors are reported with respect to the fullorder model. All the reduced-order models used the number of modes specified by Table 10. Table 13 also shows the errors $\varepsilon_{\text {avg }}$ computing using Equation 20 between the full-order model and the various versions of reduced-order models.

As shown in Table 13, the smallest errors correspond to the reduced-order model with initial time step adjustment. The largest errors correspond to the reduced-order model with projection freezing. Since the reduced-order model with projection freezing had the largest errors and the smallest speed-up factor, this acceleration technique was considered least useful. Consequently only the acceleration techniques based on database splitting and time step adjustment were combined.

Finally, the performance of ODEx was explored for a time extrapolation case where ODEx was allowed to run for an additional $0.5 \mathrm{~s}$ past the last snapshot. Time extrapolation began at $t=1.0 \mathrm{~s}$. At this time all of the field variables had reached a slowly varying state. This case tested the ability of the POD model to predict field variable values outside of the original time domain.

For the test case chosen, the number of modes used were $N p_{g}=2, N u_{g}=2, N v_{g}=5$, $N u_{s}=8, N v_{s}=5, N \epsilon_{g}=5$. This mode combination differs slightly from the one used in the standard isothermal case because it was found that this mode selection produced slightly better results for this case. Figures 18 and 19 show the reconstructed solutions of the POD model and the exact solutions of MFIX for the six field variables $p_{g}, \epsilon_{g}, u_{g}, v_{g}, u_{s}$ and $v_{s}$ respectively at $t=1.5 \mathrm{~s}$. The agreement between ODEx and MFIX is generally quite good. 
Table 13: Speed-up factors and averaged errors: ROM vs. FOM.

\begin{tabular}{lcr}
\hline & Speed-up Factor & Error, $\varepsilon_{\text {avg }}$ \\
\hline FOM & 1 & 0 \\
ROM with no acceleration & 21 & $1.882 \mathrm{E}-2$ \\
ROM with database splitting & 30 & $4.370 \mathrm{E}-2$ \\
ROM with projection freezing & 23 & 0.151818 \\
ROM with initial time step adjustment & 25 & $1.601 \mathrm{E}-2$ \\
ROM with database splitting and & & \\
$\quad$ initial time step adjustment & 114 & $6.240 \mathrm{E}-2$ \\
\hline
\end{tabular}



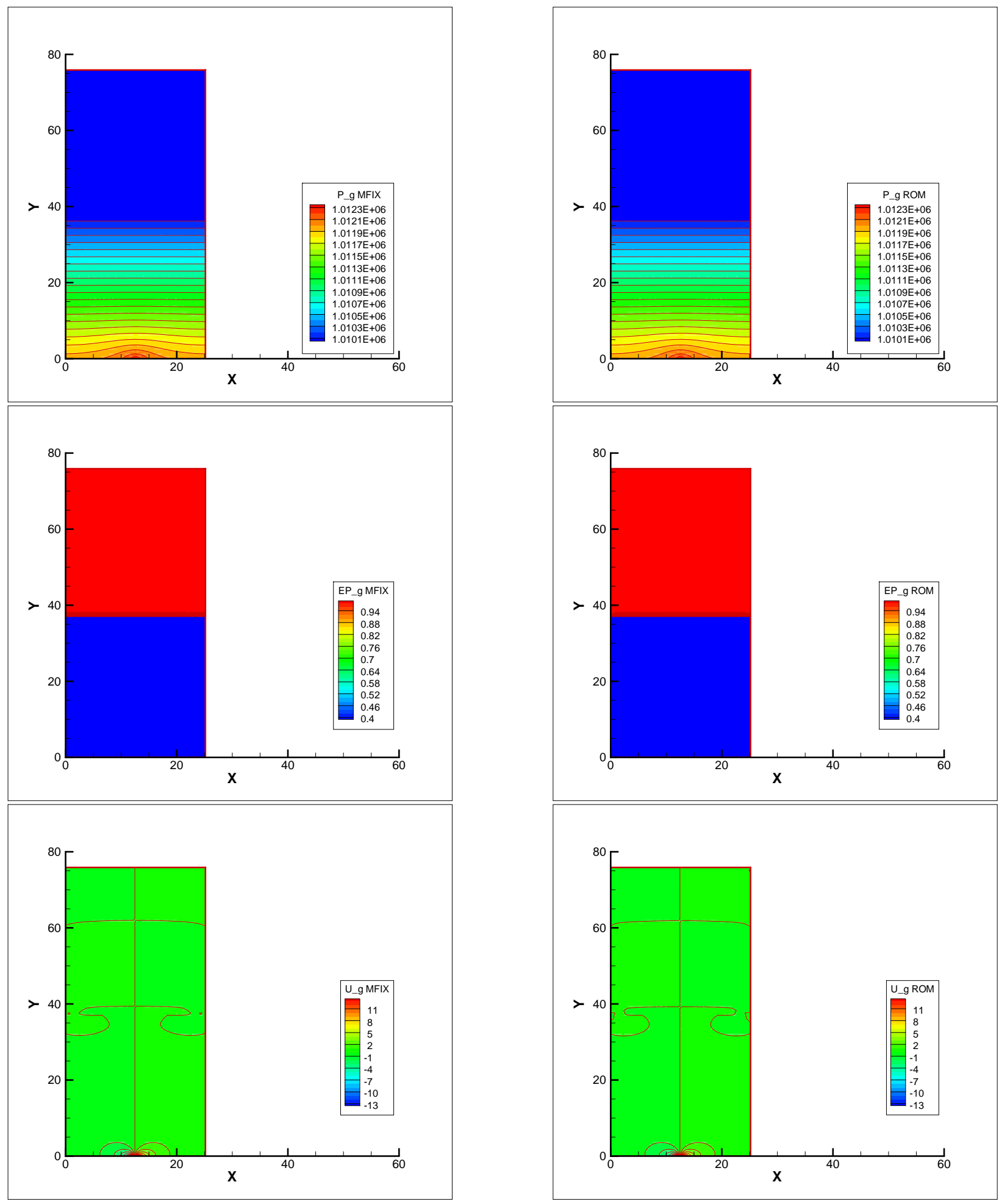

Figure 18: Gas pressure $p_{g}$, void fraction $\epsilon_{g}$ and $x$-direction gas velocity $u_{g}$ at $t=1.5 \mathrm{~s}$ : MFIX and ODEx. 

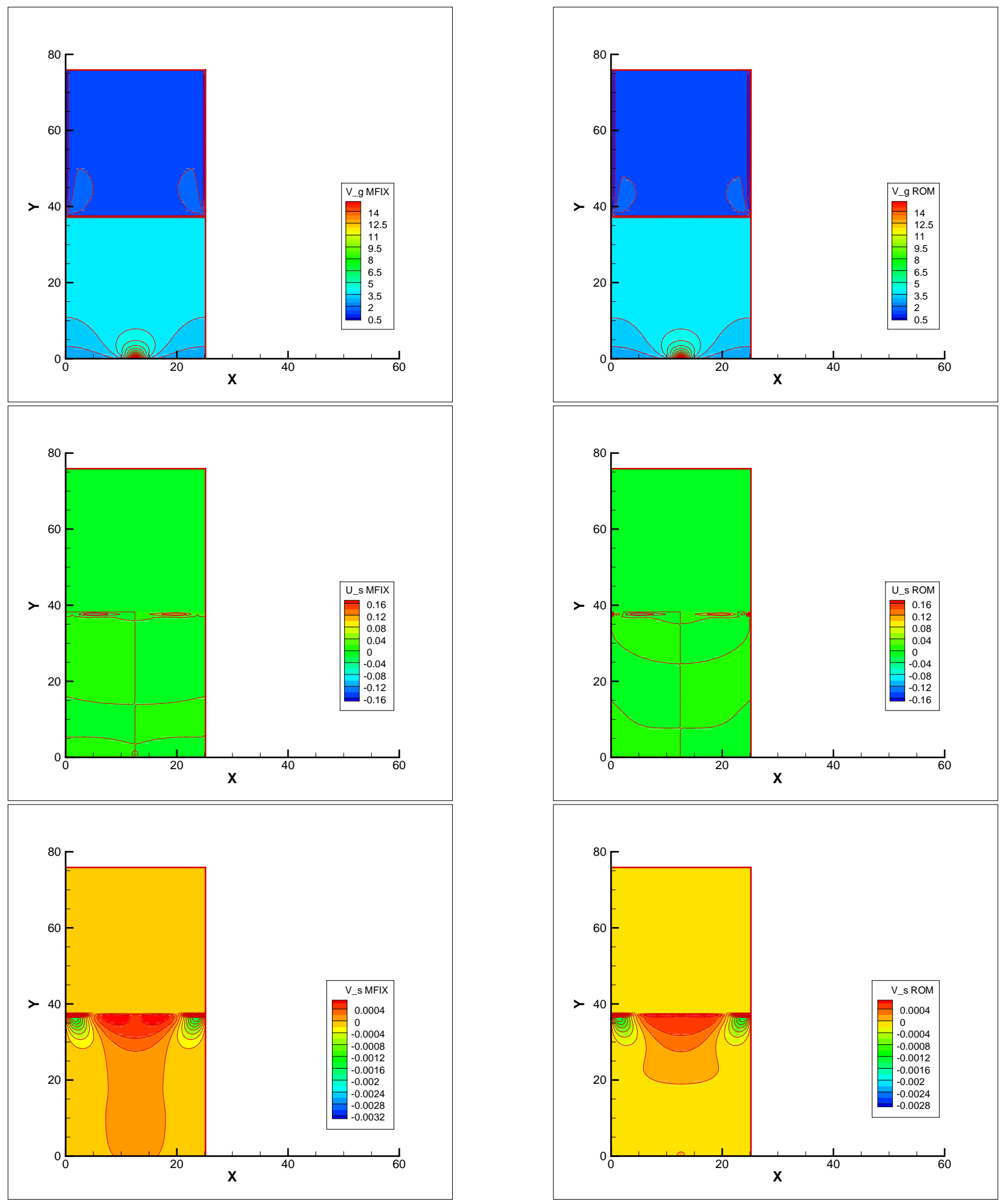

Figure 19: $y$-direction gas velocities $v_{g}$ and the $x$ - and $y$-direction solids velocities $\left(u_{s}, v_{s}\right)$ at $t=1.5 \mathrm{~s}$ : MFIX vs. ODEx. 


\subsubsection{CAPE-Open}

Several CAPE-Open (CO) interfaces have been identified for use in future integration with other simulation software (CO-LaN consortium, 2003). The six interfaces selected for ODEx are listed in Table 14. A brief summary of these interfaces and the reasons for selecting them follow.

Table 14: Interfaces identified for use in ODEx

\begin{tabular}{|c|l|l|}
\hline \hline & Interface & Selection Justification \\
\hline 1. & Identification Common Interface & Required of all components \\
2. & Parameter Common Interface & Passes input parameters into ODEx \\
3. & Error Common Interface & Communicates errors from ODEx \\
4. & Collection Common Interface & Efficiently returns lists \\
5. & Utilities Common Interface & Included for future growth \\
6. & Persistence Common Interface & Captures states for restarting simulations \\
\hline \hline
\end{tabular}

The Identification Interface provides the ODEx component with a means of supplying its name and description to other components. This interface also allows the component name and description to be set. While this isn't necessarily a critical task, every registered CO component must support this interface.

The Parameter Interface is the most relevant interface for ODEx. This interface allows for communication of both parameter values and the list of parameters being communicated. The parameters currently contained in the ODEx input file could be passed into the ODEx component using this interface.

The Error Interface will allow ODEx to communicate to the calling environment that an error has occurred. While at its heart this is a binary operation (either there is an error or there isn't) the CO standard includes several pre-defined flags for specific errors. Warnings should be handled through a separate interface which has yet to be defined within the CO standard. As an alternative solution, we suggest that warnings can be handled through the Utilities Interface, described later in this section.

The Collection Interface provides a means for a more efficient return of a list of items. For instance, a list of parameters could be retrieved this way. This interface appears to be of limited use to ODEx, but has been included due to its classification as a common interface.

The Utilities Interface functions as a catch-all for basic functionalities that are not captured elsewhere. This interface also allows the component to call upon the environment for information. There is currently no need for this interface in ODEx, but it has been included to facilitate future growth and because of its classification as a common interface.

The Persistence Interface provides a means for the simulation state to be saved periodically to facilitate future restoration. In the fully reduced version of ODEx, this is rather simple as it means capturing the time coefficients, something that is straightforward and 
requires very little storage. This interface is more useful when a hybrid version of ODEx is used and variables need to be captured at a number of spatial locations and at a number of different times.

\subsubsection{User Manual and Theory Guide}

A User Manual was written giving instructions for compiling and running ODEx. This document includes a list of permissible input parameters for the input file puv.dat. This includes parameters needed for two-dimensional, three-dimensional, isothermal, non-isothermal and granular energy cases.

A Theory Guide for ODEx was written. This document outlines the hydrodynamic model and gives the governing equations for this model. The discretization scheme is briefly described. The POD theory is given and applied to a sample problem. Finally, the governing equations for ODEx for both isothermal and non-isothermal flow, granular temperature calculation and two- and three-dimensional cases are derived.

\subsubsection{Interpolation and Extrapolation}

Previous work has explored the problem of interpolation and extrapolation of solids-phase particle diameters and fluid viscosity (Yuan et al. 2005). Additionally the extrapolation of the field variables in time has been studied (Richardson, 2007). The error in the POD approximation of the field variables has been computed as a function of time between snapshots in the snapshot database (Brenner et al, 2009). This error gives an estimate of the limits of interpolation in time on the dynamics of the flow that can be captured.

POD has been applied to a bubbling fluidized bed and the results have been analyzed in the frequency domain. The bubbling case was simulated in MFIX using the parameters given in Table 15 for the geometry shown in Fig. 1. Snapshots were captured every 0.0025 seconds, starting at $1.0 \mathrm{~s}$ and ending at $2.0 \mathrm{~s}$. This time domain was selected to capture a flow that was fully developed. When the POD basis functions were computed, it was noticed that certain basis functions for the void fraction resembled a bubble at different locations in the spatial domain as shown in Figs. 20-21. The time coefficients associated with these basis functions were then computed using the projection method and were plotted in Figs. $22-23$ as a function of time. As these time coefficients were strongly periodic, a Fourier transform was

performed to analyze the basis function in the frequency domain. The resulting frequencies are shown in Figs. 2425.

Figures 2425 show that the time coefficients associated with basis functions that have bubble-like structures have a dominant frequency of $7 \mathrm{~Hz}$. It is possible to qualitatively determine the bubbling frequency in the fluidized bed by generating an animation of the flow and counting the number of bubbles that are formed. This method shows that approximately seven bubbles are formed between $t=1.0 \mathrm{~s}$ and $t=2.0 \mathrm{~s}$. Therefore, we conclude that it is possible to determine the bubbling frequency of the flow by applying Fourier analysis to the time coefficients of the POD. Furthermore, for the case studied herein, the fourth and fifth 


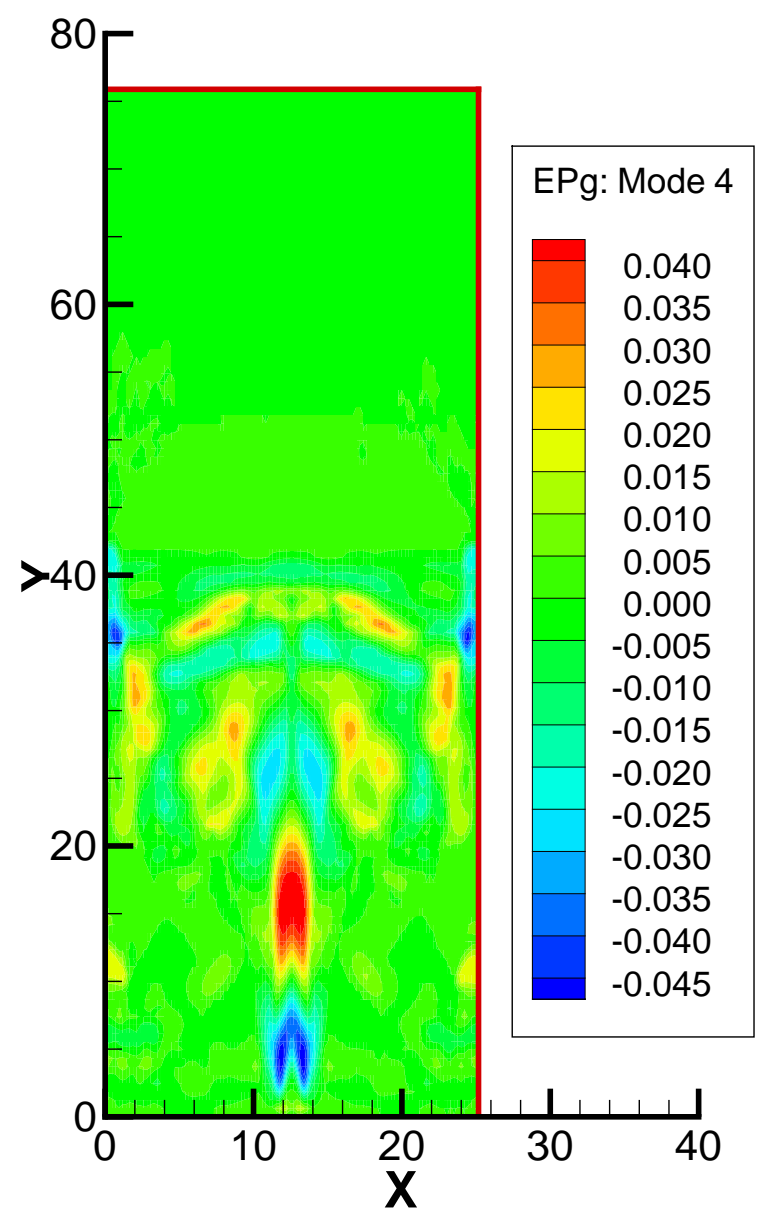

Figure 20: Fourth basis function for void frac- Figure 21: Fifth basis function for void fraction.

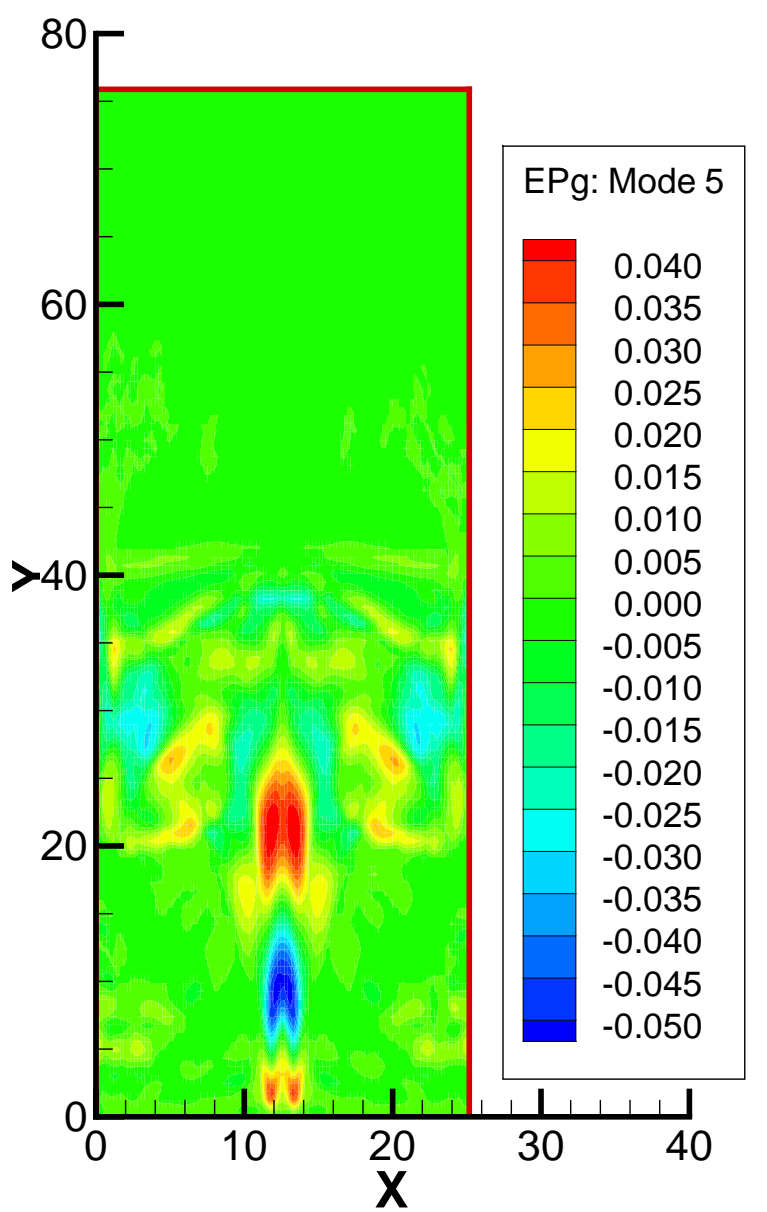




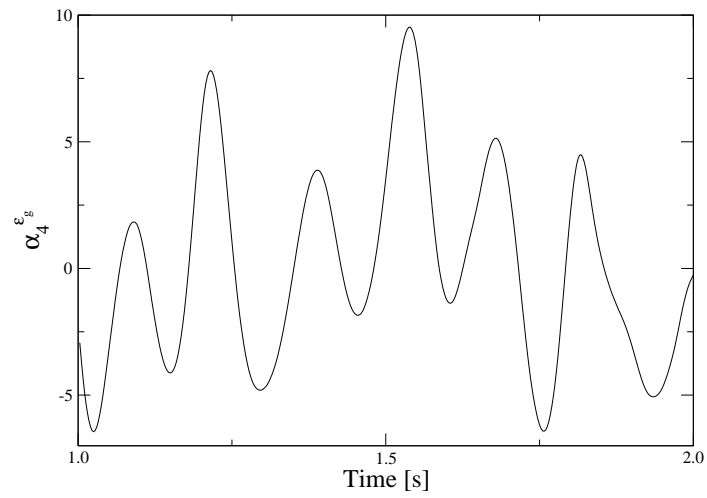

Figure 22: Fourth time coefficient for void Figure 23: Fifth time coefficient for void fracfraction.

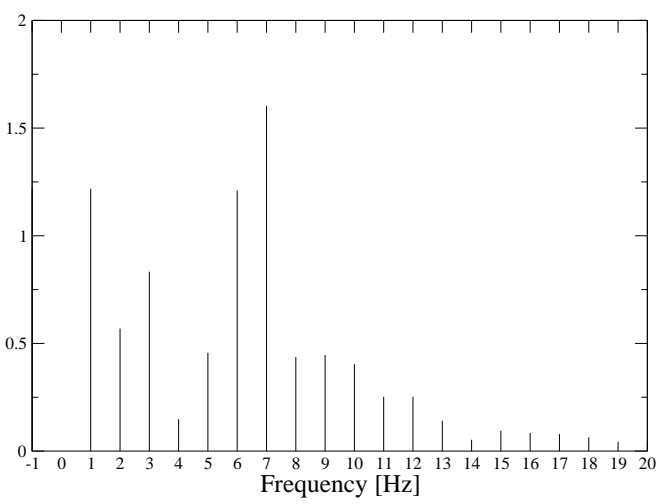

Figure 24: FFT of fourth time coefficient for void fraction.

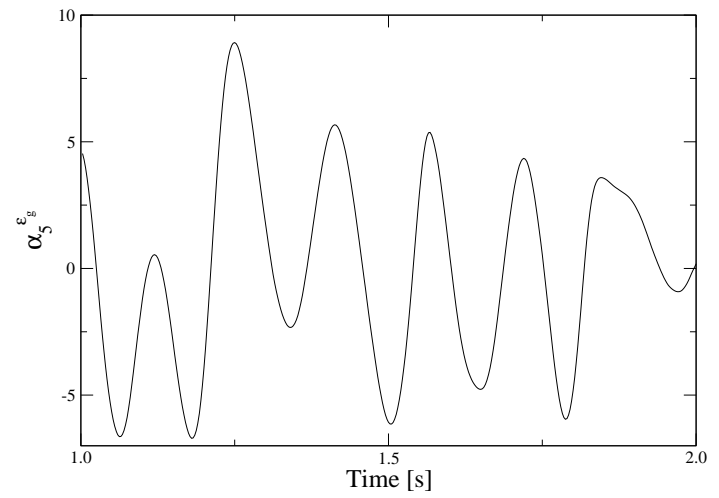

tion.

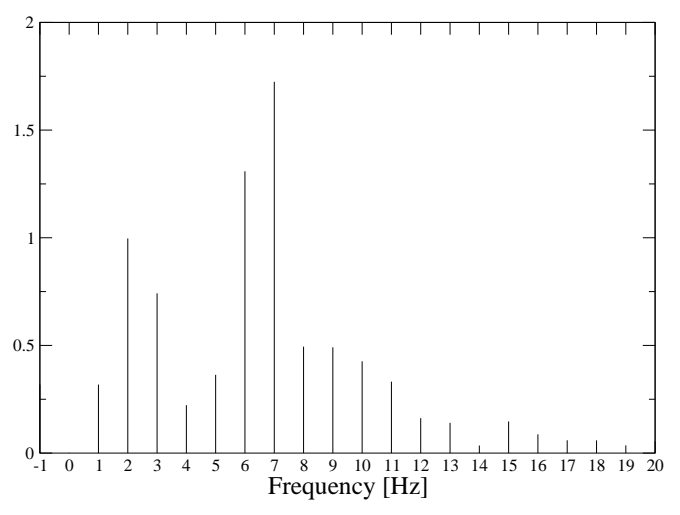

Figure 25: FFT of fifth time coefficient for void fraction. 
Table 15: Parameters of a bubbling fluidized bed (Gidaspon, 1994, p. 156).

\begin{tabular}{llcl}
\hline Parameter & Description & Units & \\
\hline$x_{\text {length }}$ & Length of the domain in $x$-direction & $\mathrm{cm}$ & 40.0 \\
$y_{\text {length }}$ & Length of the domain in $y$-direction & $\mathrm{cm}$ & 76.5 \\
$i_{\text {max }}$ & Number of cells in $x$-direction & - & 108 \\
$j_{\text {max }}$ & Number of cells in $y$-direction & - & 124 \\
$v_{1}, v_{2}$ & Gas inflow velocities & $\mathrm{cm} / \mathrm{s}$ & $577.0,53.6$ \\
$p_{g_{s}}$ & Static pressure at outlet & $\mathrm{g} / \mathrm{cm} / \mathrm{s}^{2}$ & $1.01 e^{6}$ \\
$T_{g_{0}}$ & Gas temperature & $\mathrm{K}$ & 297 \\
$\mu_{g_{0}}$ & Gas viscosity & $\mathrm{g} / \mathrm{cm} / \mathrm{s}$ & $1.8 e^{-4}$ \\
$t_{s t a r t}$ & Start time & $\mathrm{S}$ & 0.0 \\
$t_{\text {stop }}$ & Stop time & $\mathrm{S}$ & 5.0 \\
$\rho_{s}$ & Particle density & $\mathrm{g} / \mathrm{cm}^{3}$ & 2.42 \\
$D_{p}$ & Particle diameter & $\mathrm{cm}$ & 0.08 \\
$h_{s 0}$ & Initial height of packed bed & $\mathrm{cm}$ & 29.2 \\
$\epsilon_{g}^{*}$ & Initial void fraction of packed bed & - & 0.4 \\
\hline
\end{tabular}

POD modes capture the structure of the bubble.

\subsubsection{Dissemination of Research Results}

The paper titled "Practical Aspects of the Implementation of Proper Orthogonal Decomposition" was presented at the 47th AIAA Aerospace Sciences Meeting on January 7th, 2009 (Brenner et an, 2009). This paper described our investigation of the effect of time sampling on POD error and a brief study of the necessity of including inter-variable crosscorrelation terms in the computation of the POD basis functions. In the near future this paper will be submitted to a peer-reviewed journal for archival publication.

The paper titled "Acceleration techniques for reduced-order models based on proper orthogonal decomposition" was published in in the Journal of Computational Physics, volume 227 (Cizmas et al, 2008). This paper presents the ROM based on POD used to solve the void fraction. It also details the various acceleration methods that have been developed to reduce the computational time required by ODEx.

A Paper titled "Augmented Proper Orthogonal Decomposition for Problems with Moving Discontinues" has been submitted to the journal Powder Technology for publication in a special issue. This paper presents our efforts to adapt the POD method to capture moving discontinuities. It also includes a numerical algorithm for identifying the edges of bubbles. 


\subsubsection{Granular Energy}

Granular temperature, $\Theta_{m}$, is defined in the kinetic granular theory (that is based on an analogy of the kinetic theory of dense gases) to be proportional to the mean quadratic velocity of the random motion of grains:

$$
\Theta_{m}=\frac{1}{3}\left\langle C_{m}^{2}\right\rangle
$$

where $C_{m}$ is the velocity of the chaotic motion of grains and the symbol \langle\rangle denotes an ensemble average. The granular energy, that is, the fluctuating energy per unit mass due to granular random motion is

$$
E_{\Theta_{m}}=\frac{1}{2}\left\langle C_{m}^{2}\right\rangle=\frac{3}{2} \Theta_{m}
$$

Consequently, granular energy is proportional to granular temperature (Eyamlal et an., 1994, p. 27).

Currently MFIX provides the option to use an algebraic expression to solve for the granular temperature:

$$
\Theta_{m}=\left(\frac{-K_{1 m} \varepsilon_{m} D_{m i i}+\sqrt{K_{1 m}^{2} D_{m i i}^{2} \varepsilon_{m}^{2}+4 K_{4 m} \varepsilon_{m}\left[K_{2 m} D_{m i i}^{2}+2 K_{3 m} D_{m i j} D_{m i j}\right]}}{2 \varepsilon_{m} K_{4 m}}\right)^{2}
$$

where expressions for $K_{i m}$ are given in (Benyahia et a1, 2007, p. 5). This algebraic model of the granular temperature has been implemented and tested in ODEx.

Equation (29) is a highly simplified version of the following PDE for one solid phase (Benyahia et al., 2007, p. 2):

$$
\frac{3}{2} \varepsilon_{s} \rho_{s}\left[\frac{\partial \Theta_{m}}{\partial t}+U_{s j} \frac{\partial \Theta_{m}}{\partial x_{j}}\right]=\frac{\partial}{\partial x_{i}}\left(\kappa_{s} \frac{\partial \Theta_{m}}{\partial x_{i}}\right)+\tau_{s i j} \frac{\partial U_{s i}}{\partial x_{j}}+\Pi_{s}-\varepsilon_{s} \rho_{s} J_{s}
$$

where production is equated to dissipation in Equation (30) to derive Equation (29). Equation (30) can be discretized to the following form:

$$
a_{p}^{\Theta_{m}} \Theta_{m, p}=\sum_{n b} a_{n b}^{\Theta_{m}} \Theta_{m, n b}+b_{p}^{\Theta_{m}}
$$

where the $a_{n b}^{\Theta_{m}}, a_{p}^{\Theta_{m}}, b_{p}^{\Theta_{m}}$ coefficients are defined in the MFIX Numerical Technique document in a manner similar to the energy and species balance equations (Syamla, 1998, pp. 15-21). 
The granular energy is approximated using the basis functions of $\Theta_{m}, \phi_{i}^{\Theta_{m}}$

$$
\Theta_{m}(x, t) \cong \sum_{j=0}^{m^{\Theta_{m}}} \alpha_{j}^{\Theta_{m}}(t) \phi_{j}^{\Theta_{m}}(x)
$$

where $m^{\Theta_{m}}$ is the number of granular energy modes kept in the approximation, including the average mode.

Substituting Equation (32) into Equation (31) yields

$$
a_{p}^{\Theta_{m}} \sum_{j=0}^{m^{\Theta_{m}}} \alpha_{j}^{\Theta_{m}} \phi_{j_{p}}^{\Theta_{m}}=\sum_{n b} a_{n b}^{\Theta_{m}} \sum_{j=0}^{m^{\Theta_{m}}} \alpha_{j}^{\Theta_{m}} \phi_{j_{n b}}^{\Theta_{m}}+b_{p}^{\Theta_{m}}
$$

The Galerkin projection of Equation (33) onto the basis functions $\phi_{i}^{\Theta_{m}}$ yields

$$
a_{p}^{\Theta_{m}}\left(\sum_{j=0}^{m^{\Theta_{m}}} \alpha_{j}^{\Theta_{m}} \phi_{j_{p}}^{\Theta_{m}}, \phi_{i}^{\Theta_{m}}\right)=\sum_{n b} a_{n b}^{\Theta_{m}}\left(\sum_{j=0}^{m^{\Theta_{m}}} \alpha_{j}^{\Theta_{m}} \phi_{j_{n b}}^{\Theta_{m}}, \phi_{i}^{\Theta_{m}}\right)+\left(b_{p}^{\Theta_{m}}, \phi_{i}^{\Theta_{m}}\right), \quad i=0, m^{\Theta_{m}} .
$$

Equation (34) can be rearranged as

$$
\sum_{j=0}^{m^{\Theta_{m}}} \alpha_{j}^{\Theta_{m}}\left[a_{p}^{\Theta_{m}}\left(\phi_{j_{p}}^{\Theta_{m}}, \phi_{i}^{\Theta_{m}}\right)-\sum_{n b} a_{n b}^{\Theta_{m}}\left(\phi_{j_{n b}}^{\Theta_{m}}, \phi_{i}^{\Theta_{m}}\right)\right]=\left(b_{p}^{\Theta_{m}}, \phi_{i}^{\Theta_{m}}\right), \quad i=0, m^{\Theta_{m}} .
$$

The system of linear equations (35) can be written as

$$
\tilde{\mathcal{A}}^{\Theta_{m}} \alpha^{\Theta_{m}}=\tilde{\mathcal{B}}^{\Theta_{m}}
$$

where

$$
\tilde{\mathcal{A}}_{i j}^{\Theta_{m}}=\left(\left(a_{p}^{\Theta_{m}} \phi_{j}^{\Theta_{m}}-\sum_{n b} a_{n b}^{\Theta_{m}} \phi_{j, n b}^{\Theta_{m}}\right), \phi_{i}^{\Theta_{m}}\right)
$$

and

$$
\tilde{\mathcal{B}}_{i}^{\Theta_{m}}=\left(b_{p}^{\Theta_{m}}, \phi_{i}^{\Theta_{m}}\right) .
$$

The dimensions of $\tilde{\mathcal{A}}$ and $\tilde{\mathcal{B}}$ are $m^{\Theta_{m}} \times m^{\Theta_{m}}$ and $m^{\Theta_{m}} \times 1$, respectively.

The algorithm for the reduced granular energy was implemented into ODEx using Equation (36) and tested. The case in Table 2 was modified to give the solids an initial granular energy of $10 \frac{\mathrm{cm}^{2}}{\mathrm{~s}^{2}}$. This was then used to verify the algorithm. Figure 26 shows the granular energy at $t=1.0 \mathrm{~s}$ for MFIX and ODEx. The number of modes used in the POD reconstruc- 


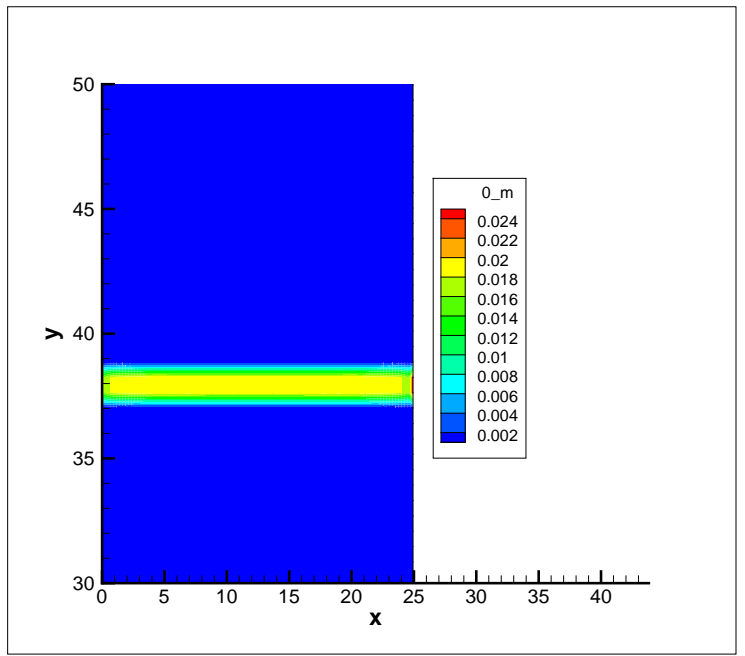

(a)

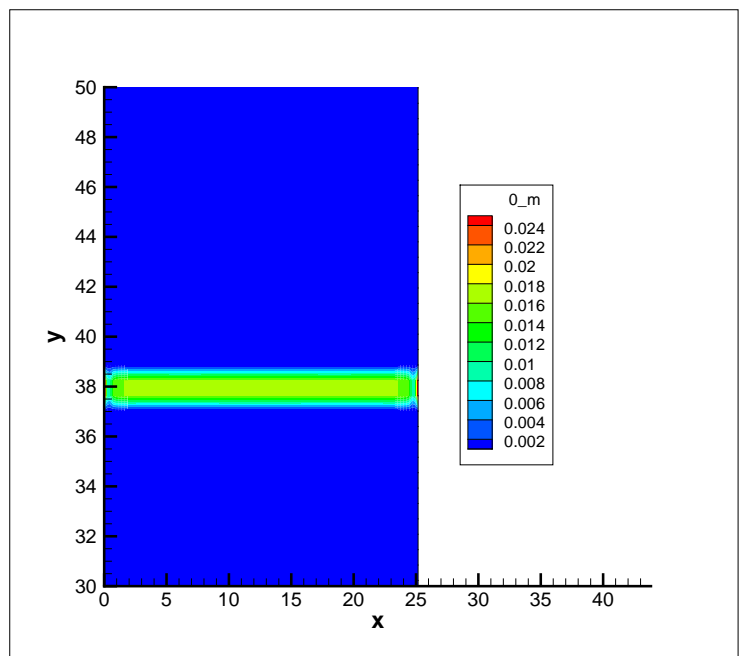

(b)

Figure 26: Contour plots of granular energy at $t=1 \mathrm{~s}$ : (a) MFIX, and (b) ODEx.

tion were 2,2,6,9,9,7 and 3 for the pressure, $x$-direction gas, $y$-direction gas, $x$-direction solids, and $y$-direction solids velocities, void fraction, and the granular energy, respectively. There is very good agreement between the solutions of the full-order and reduced-order model. The first two time coefficients for the granular energy are presented in Figure 27. The first time coefficient is closely approximated by ODEx, while the second one tends to diverge as the solution is moved forward in time. The first mode of granular energy carries over 99 percent of the energy, and therefore the solution is not greatly affected by the divergence of the second time coefficient. Also, granular energy is closely coupled to the other field variables, particularly the solids velocities and void fraction. Good approximations of these field variables tend to lead to better approximations of the granular energy.

\subsubsection{Three-Dimensional Algorithm for ROM}

MFIX models the $z$-direction gas and solids velocities as

$$
a_{p}^{w_{\ell}} w_{\ell}+\sum_{n b} a_{n b}^{w_{\ell}} w_{\ell}=b_{p}^{w_{\ell}}
$$

where the $a_{n b}^{w_{\ell}}, a_{p}^{w_{\ell}}, b_{p}^{w_{\ell}}$ coefficients are defined in the MFIX Numerical Technique document (5yamla, 1998, pp. 15-21) and $\ell$ represents the phase, either gas or solids. The velocity can then be approximated using the POD basis functions as

$$
w_{\ell}(x, t)=\phi_{0}^{w_{\ell}}(x)+\sum_{j=1}^{m^{w_{\ell}}} \alpha_{j}^{w_{\ell}}(t) \phi_{j}^{w_{\ell}}(x),
$$




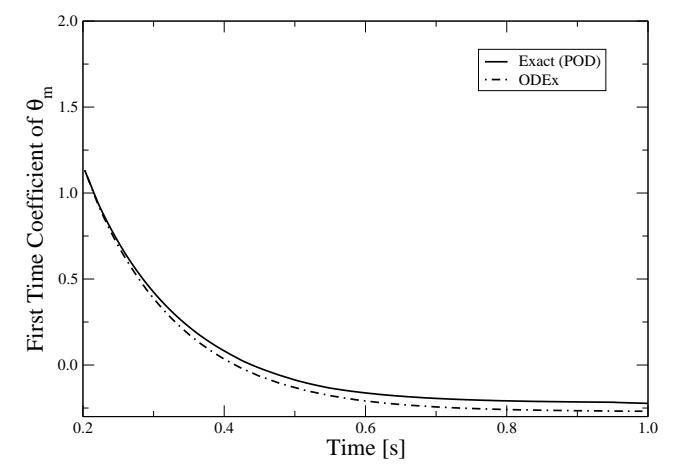

(a)

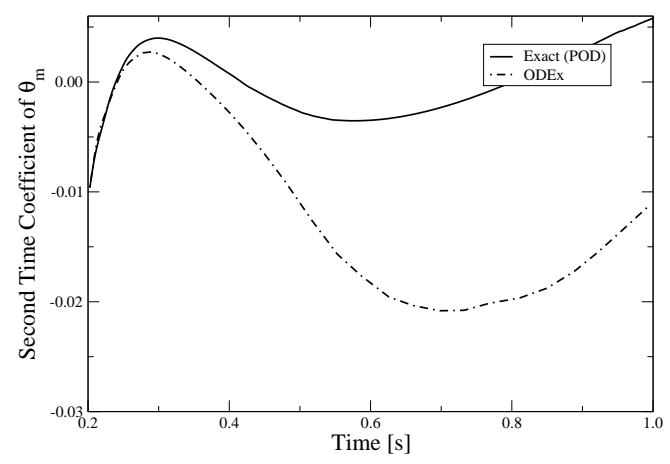

(b)

Figure 27: Comparison of time coefficients for granular energy: (a) $\alpha_{1}^{\Theta_{m}}$, and (b) $\alpha_{2}^{\Theta_{m}}$.

where $m^{w_{\ell}}$ is the number of $z$-direction velocity modes kept in the approximation. Substituting Equation (38) into Equation (37), projecting the result onto the POD basis functions and collecting the time coefficient terms on the left hand side of the equation,

$$
\tilde{\mathcal{A}}^{w_{\ell}} \alpha^{w_{\ell}}=\tilde{\mathcal{B}}^{w_{\ell}}
$$

where

$$
\begin{aligned}
& \tilde{\mathcal{A}}_{i j}^{w_{\ell}}=\left(\left(a_{p}^{w_{\ell}} \phi_{j}^{w_{\ell}}+\sum_{n b} a_{n b}^{w_{\ell}} \phi_{j, n b}^{w_{\ell}}\right), \phi_{i}^{w_{\ell}}\right), \\
& \tilde{\mathcal{B}}_{i}^{w_{\ell}}=\left(b_{p}^{w_{\ell}}-a_{p}^{w_{\ell}}-\sum_{n b} a_{n b}^{w_{\ell}} \phi_{0, n b}^{w_{\ell}}, \phi_{i}^{w_{\ell}}\right) .
\end{aligned}
$$

The dimensions of $\tilde{\mathcal{A}}$ and $\tilde{\mathcal{B}}$ are $m^{w_{\ell}} \times m^{w_{\ell}}$ and $m^{w_{\ell}} \times 1$, respectively.

\subsubsection{D Algorithm Implementation}

The reduced-order models for the $z$-direction gas and solids velocities have been implemented in ODEx. Additionally, the other field variables now model the contribution of the top and bottom neighbor cells as fluxes through the top and bottom faces in the three-dimensional case. A logical variable was used to preserve the ability of ODEx to model two-dimensional flows. 


\subsubsection{Testing of ROM vs. FOM}

A three-dimensional test case was developed to test the ROM. This test case used the same parameters specified in Table $\mathrm{Z}$ and the geometry shown in Fig. 1 was extruded in the $z$ direction. For this case the central jet was a slot oriented in the $z$-direction. MFIX was used to model the flow and PODDEC was used to extract the basis functions. The time coefficients computed by ODEx are shown in Figures 28 and 29 where they are compared to the best-case time coefficients computed by PODDEC. The agreement is generally very good.

\subsubsection{D Granular Energy and Temperature Algorithm}

The algorithms used to compute the granular energy and gas and solids temperatures in ODEx were extended to a third dimension. This algorithm now includes the fluxes through the top and bottom of the control volume. As with the other field variables, a logical variable was used to allow the solution of $2 \mathrm{D}$ flows as well. 

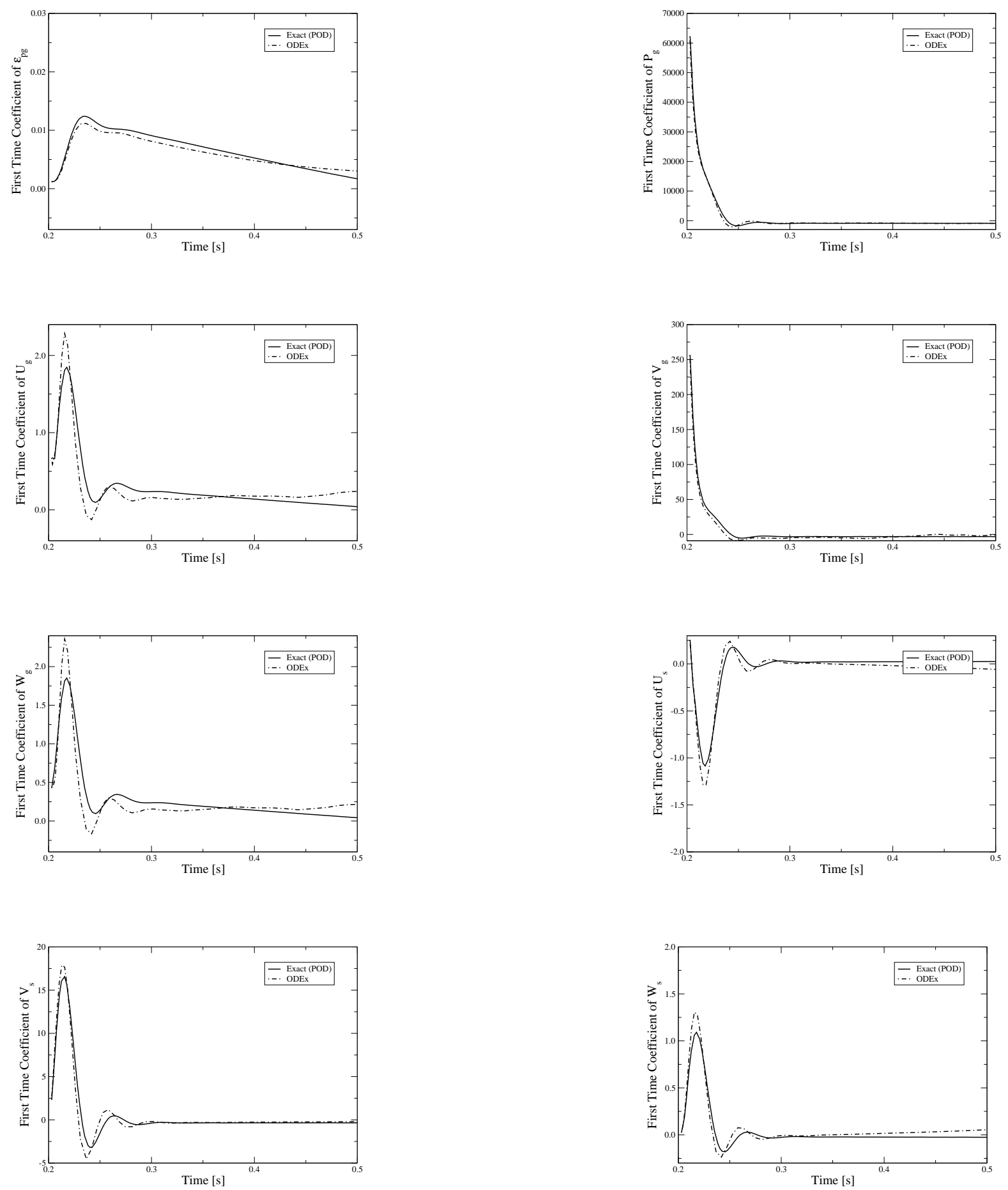

Figure 28: Time history of the first time coefficient calculated using PODDEC and ODEx for the $3 \mathrm{D}$ test case. 

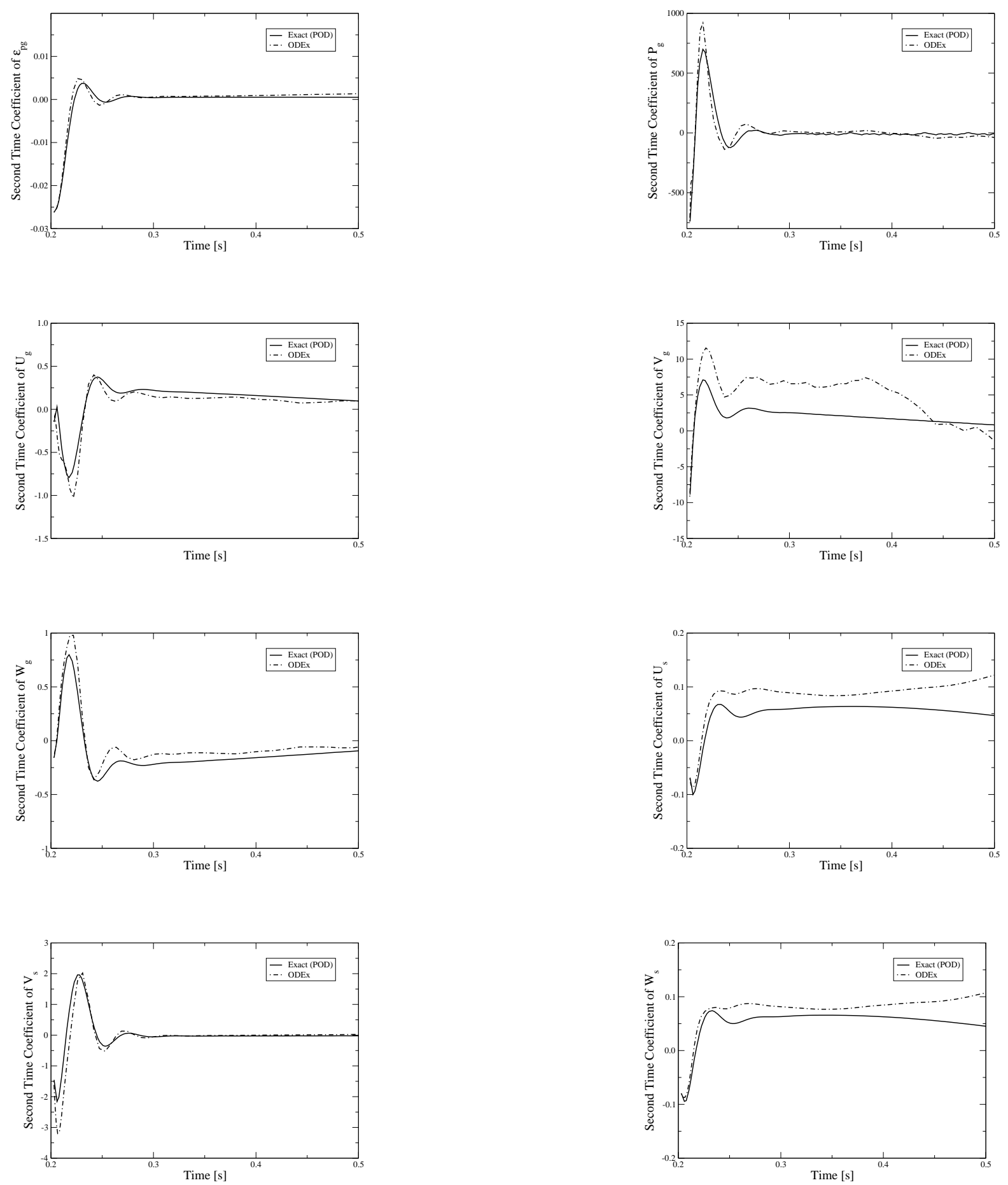

Figure 29: Time history of the second time coefficient calculated using PODDEC and ODEx for the $3 \mathrm{D}$ test case. 


\subsection{Additional Accomplishments}

Several additional accomplishments were made during this project. Included is a study of the effect of snapshot distribution on the POD basis functions. Also presented is an attempt to compute coupled POD basis functions.

The current implementation of the reduced-order solids volume fraction correction equation is unable to simulate flow with bubbles, which represent moving discontinuities in the void fraction. Various strategies for adapting our model to capture moving discontinuities are presented. These were not implemented due to time constraints, but they include a method for augmenting the POD basis functions with discontinuity modes and a method for using point modes to recover the full-order model in regions of the flow where the ROM produced unphysical results. A numerical method for identifying the edges of bubbles is also detailed.

\subsubsection{Snapshot Distribution Study}

The literature makes no claim as to the optimal distribution of snapshots. It is logical to state that the important dynamics of the flow must be contained in the snapshot ensemble if they are to be captured by the basis functions. To the best of our knowledge, there has been no study to determine the optimal placement in time of the snapshots.

Two studies were carried out. In the first, the snapshot sampling frequency was progressively increased and the reconstructed solution was compared against a reference solution, $u(x, t)$, consisting of a snapshot database containing 4823 snapshots. This represented the output of MFIX at every time step. It is therefore reasonable to assume that this solution captures all of the relevant flow dynamics. The error for each snapshot database is then defined by

$$
\varepsilon_{m}=\left\langle\left\|u\left(x, t_{i}\right)-\sum_{k=0}^{m} \alpha_{k}\left(t_{i}\right) \varphi_{k}(x)\right\|^{2}\right\rangle
$$

In the second study, the snapshots were redistributed so that the snapshot frequency was increased during periods of transience, while keeping the total number of snapshots as close to 200 as possible. The solution computed from the reconstruction of these data was then compared against the reference solution, using Equation (39). The increase in snapshot frequency during transience was accomplished in two ways. The first method used a larger constant sampling rate in the transient period and a smaller constant sampling rate in the quasi-steady period. This is shown in Fig. 30 where it is referred to as method 1. In the second method, a logarithmic function was developed to give a continuously varying sampling rate, with snapshots collected less frequently as the simulation progressed.

To obtain this logarithmic distribution, MFIX was run to generate an evenly spaced snapshot database with 320 realizations. ODEx was then used to find the relationship 


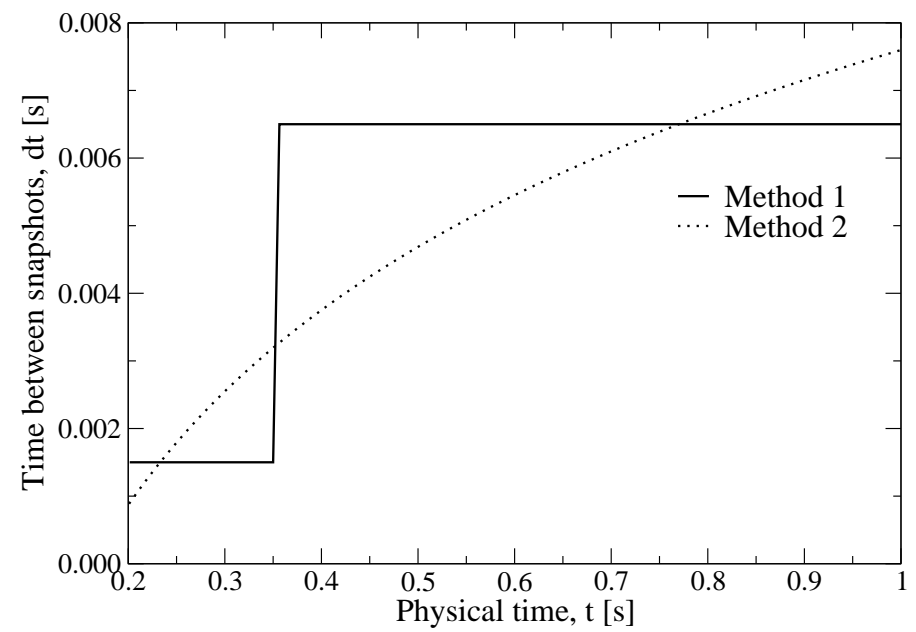

Figure 30: Time between snapshots as a function of physical time, t, for method 1: constant $\mathrm{dt}$ on each interval and method 2 : continuously varying $\mathrm{dt}$.

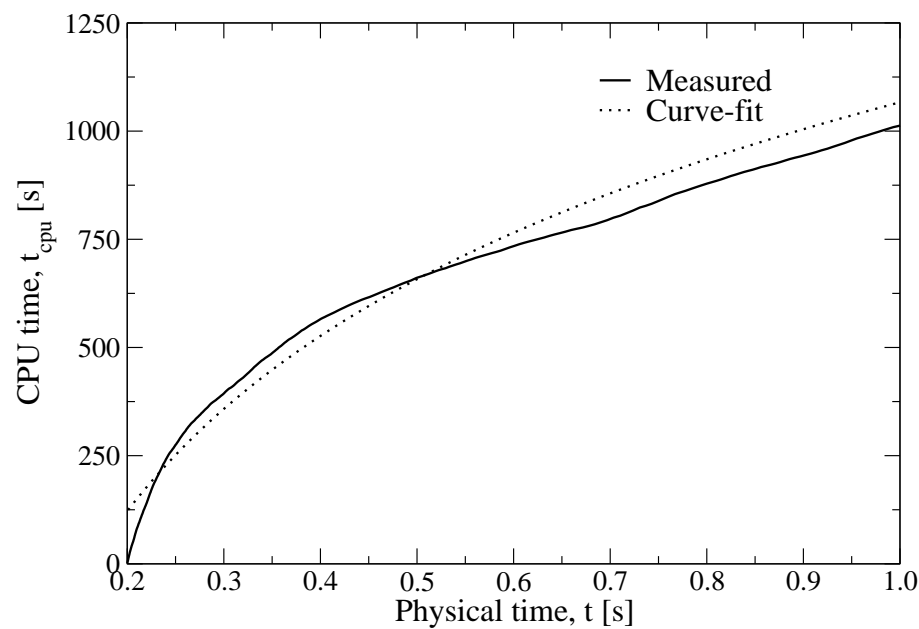

Figure 31: Relationship between physical time and CPU time in ODEx, with curve fit. 
between physical time, $t$, and CPU time, $t_{c p u}$ shown in Fig. 31. These data were selected as a useful measure of the complexity of the flow. A curve fit to these data was expressed by

$$
t_{\text {сpu }}(t)=588.14 \ln (t)+1071.0 .
$$

This equation was then scaled to obtain a function that gave as close to 200 snapshots in the computation region as possible, while still maintaining the shape of the curve. The time between snapshots was given by

$$
d t(t)=0.00419 \ln (t)+0.00763
$$

and is shown as method 2 in Fig. 30. In practice, this meant that MFIX was run again and the first snapshot was collected at $t=0.2 \mathrm{~s}$. The time between snapshots was then calculated based on this $t$ and the next sampling point was determined. The timing of the third snapshot could then be found using the time location of the second sampling point. This was continued until the end of the simulation at $t=1.0 \mathrm{~s}$ and resulted in the collection of 197 snapshots. This method is somewhat cumbersome, requiring MFIX to be run twice, and a more convenient method may exist. One possibility is to perform an interpolation on the data in the first snapshot database to generate a second snapshot database with logarithmic spacing in time.

The errors computed using Equation (39) for the snapshot distribution study are given in Table 16. To obtain these error values, the solution was reconstructed using the number of modes given in Table 17. The reconstructed solutions were linearly interpolated to find values of the field variables at the proper spacing for comparison with the reference solution at all 4283 time locations.

As Table 16 shows, the error tended to decrease as additional snapshots were collected and added to the computation of the basis functions. Note that the case with 200 snapshots has been presented out of sequence for easier comparison with the two unevenly distributed cases.

In the the unevenly distributed cases, where the snapshots were collected more frequently in the areas of flow transience, the errors improved drastically as compared to snapshots that were evenly spaced with a similar number of snapshots collected. In the study, the best results for gas pressure were obtained using a logarithmic distribution of 197 snapshots. These results were generally better than even the case where 800 evenly distributed snapshots were used.

Using a distribution that was constant in the transient region and another, less frequent, constant distribution in the quasi-steady region produced results that were nearly as good as the logarithmic distribution. This second case is easier in practice, as the function that gave the logarithmic distribution in time required a curve fit to data calculated with the ROM and was therefore not known a priori. As a result of this complication, the method using different constant sampling rates at different times is recommended. 
Table 16: POD reconstruction error using Equation (39) for various snapshot distributions

\begin{tabular}{lccccccc}
\hline $\begin{array}{l}\text { Time between } \\
\text { snapshots, dt, }[\mathrm{s}]\end{array}$ & No. of & & & \multicolumn{5}{c}{ Error, $\epsilon$} \\
\hline 0.1 & snapshots & $u_{g}$ & $v_{g}$ & $u_{s}$ & $v_{s}$ & $\epsilon_{g}$ & $p_{g}$ \\
0.075 & 8 & 0.166 & 48.628 & $4.324 \mathrm{E}-003$ & 0.584 & $5.737 \mathrm{E}-006$ & $3.424 \mathrm{E}+006$ \\
0.05 & 12 & 0.167 & 30.761 & $5.840 \mathrm{E}-003$ & 0.518 & $5.305 \mathrm{E}-006$ & $1.977 \mathrm{E}+006$ \\
0.025 & 16 & 0.166 & 58.420 & $6.459 \mathrm{E}-003$ & 0.558 & $3.204 \mathrm{E}-006$ & $3.978 \mathrm{E}+006$ \\
0.01 & 32 & 0.163 & 21.339 & $6.328 \mathrm{E}-003$ & 0.478 & $6.342 \mathrm{E}-006$ & $1.240 \mathrm{E}+006$ \\
0.0075 & 80 & 0.161 & 14.513 & $1.539 \mathrm{E}-003$ & 0.195 & $4.507 \mathrm{E}-007$ & $6.779 \mathrm{E}+005$ \\
0.005 & 108 & 0.160 & 1.165 & $5.346 \mathrm{E}-004$ & $1.149 \mathrm{E}-002$ & $1.438 \mathrm{E}-008$ & $3.883 \mathrm{E}+004$ \\
0.0025 & 160 & 0.161 & 1.709 & $1.052 \mathrm{E}-003$ & $1.401 \mathrm{E}-002$ & $1.578 \mathrm{E}-008$ & $9.977 \mathrm{E}+004$ \\
0.001 & 320 & 0.160 & 0.812 & $3.675 \mathrm{E}-004$ & $2.059 \mathrm{E}-003$ & $2.014 \mathrm{E}-009$ & $9.807 \mathrm{E}+003$ \\
\hline 0.004 & 800 & 0.160 & 0.247 & $1.315 \mathrm{E}-004$ & $1.937 \mathrm{E}-004$ & $1.781 \mathrm{E}-009$ & $1.287 \mathrm{E}+003$ \\
0.0015 and 0.0065 & 200 & 0.161 & 0.981 & $1.533 \mathrm{E}-003$ & $9.015 \mathrm{E}-003$ & $4.617 \mathrm{E}-009$ & $4.406 \mathrm{E}+004$ \\
varied from 9.2E-4 to 0.0075 & 197 & 0.165 & 0.241 & $1.439 \mathrm{E}-004$ & $3.525 \mathrm{E}-004$ & $3.372 \mathrm{E}-008$ & $1.254 \mathrm{E}+003$ \\
\hline
\end{tabular}

Table 17: Number of modes used and their symbols.

\begin{tabular}{lcc}
\hline Field variable & Symbol & No. of modes \\
\hline Gas pressure & $N_{p_{g}}$ & 2 \\
Solids volume fraction & $N_{\epsilon s}$ & 7 \\
$u$ gas velocity & $N_{u g}$ & 2 \\
$v$ gas velocity & $N_{v_{g}}$ & 5 \\
$u$ solids velocity & $N_{u s}$ & 8 \\
$v$ solids velocity & $N_{v_{s}}$ & 6 \\
\hline
\end{tabular}

\subsubsection{Composition of the Autocorrelation Matrix}

Another topic of study was the optimal composition of the autocorrelation matrix, $\overline{\bar{R}}$. Two approaches were explored, a split approach and a coupled approach.

Recall that proper orthogonal decomposition is a procedure for extracting time-independent basis functions from data that are dependent on both time and space. Consider a set of discrete snapshots represented by $u\left(x, t_{i}\right)$ for $i=1, \ldots, M$. These observations are assumed to form a linear, finite-dimensional Hilbert space $L^{2}$ on a spatial domain $D$. The basis functions, $\left\{\varphi_{k}(x)\right\}$ are computed such that the reconstruction Equation (18) is optimal in the sense that the average least-square truncation error given by Equation $(\overline{B 9})$ is a minimum for any given number $m \leq M$. Herein $\|\cdot\|$ denotes the $L^{2}$-norm given by $\|f\|=(f, f)^{\frac{1}{2}}$, where $($,$) denotes the Euclidean inner product. \langle\cdot\rangle$ denotes an ensemble average over the 
number of observations $\langle f\rangle=\sum_{i=1}^{M} f\left(x, t_{i}\right) / M$.

This optimization problem can be recast as an eigenvalue problem with (Holmes et a1., 1996)

$$
\int_{D}\left\langle u(x) u^{*}(y)\right\rangle \varphi(y) d y=\lambda \varphi(x) .
$$

Then, the optimal basis functions can be calculated as the eigenfunctions of Equation (40). In the finite-dimensional case this reduces to finding the eigenvectors of the autocorrelation matrix defined by

$$
\overline{\bar{R}}(\mathbf{x}, \mathbf{y})=\sum_{i=1}^{M} \mathbf{u}\left(\mathbf{x}, t_{i}\right) \mathbf{u}^{T}\left(\mathbf{y}, t_{i}\right) / M
$$

It is this autocorrelation matrix, $\overline{\bar{R}}$, that our study is concerned with.

In the coupled approach the field variables were concatenated into a single variable that was used to compute a single autocorrelation matrix (Kirby et al., [1999). The concatenated variable was:

$$
u\left(x, t_{i}\right)=\left(\begin{array}{c}
u_{g}\left(x, t_{i}\right) \\
v_{g}\left(x, t_{i}\right) \\
u_{s}\left(x, t_{i}\right) \\
v_{s}\left(x, t_{i}\right) \\
\epsilon_{g}\left(x, t_{i}\right) \\
p_{g}\left(x, t_{i}\right)
\end{array}\right)
$$

This new variable was then used directly in Equation (41) and the autocorrelation matrix was given by

$$
\{\overline{\bar{R}}\}=\left(\begin{array}{cccc}
\overline{\bar{R}}_{11} & \overline{\bar{R}}_{12} & \ldots & \overline{\bar{R}}_{1 N} \\
\overline{\bar{R}}_{21} & \overline{\bar{R}}_{22} & \ldots & \overline{\bar{R}}_{2 N} \\
\vdots & \vdots & \ddots & \vdots \\
\overline{\bar{R}}_{N 1} & \overline{\bar{R}}_{N 2} & \ldots & \overline{\bar{R}}_{N N}
\end{array}\right)
$$

where

$$
\left\{\overline{\bar{R}}_{i j}\right\}=\left(\begin{array}{cccccc}
\left\langle p_{g i} * p_{g j}\right\rangle & \left\langle\epsilon_{g i} * p_{g j}\right\rangle & \left\langle u_{g i} * p_{g j}\right\rangle & \left\langle v_{g i} * p_{g j}\right\rangle & \left\langle u_{s i} * p_{g j}\right\rangle & \left\langle v_{s i} * p_{g j}\right\rangle \\
\left\langle p_{g i} * \epsilon_{g j}\right\rangle & \left\langle\epsilon_{g i} * \epsilon_{g j}\right\rangle & \left\langle u_{g i} * \epsilon_{g j}\right\rangle & \left\langle v_{g i} * \epsilon_{g j}\right\rangle & \left\langle u_{s i} * \epsilon_{g j}\right\rangle & \left\langle v_{s i} * \epsilon_{g j}\right\rangle \\
\left\langle p_{g i} * u_{g j}\right\rangle & \left\langle\epsilon_{g i} * u_{g j}\right\rangle & \left\langle u_{g i} * u_{g j}\right\rangle & \left\langle v_{g i} * u_{g j}\right\rangle & \left\langle u_{s i} * u_{g j}\right\rangle & \left\langle v_{s i} * u_{g j}\right\rangle \\
\left\langle p_{g i} * v_{g j}\right\rangle & \left\langle\epsilon_{g i} * v_{g j}\right\rangle & \left\langle u_{g i} * v_{g j}\right\rangle & \left\langle v_{g i} * v_{g j}\right\rangle & \left\langle u_{s i} * v_{g j}\right\rangle & \left\langle v_{s i} * v_{g j}\right\rangle \\
\left\langle p_{g i} * u_{s j}\right\rangle & \left\langle\epsilon_{g i} * u_{s j}\right\rangle & \left\langle u_{g i} * u_{s j}\right\rangle & \left\langle v_{g i} * u_{s j}\right\rangle & \left\langle u_{s i} * u_{s j}\right\rangle & \left\langle v_{s i} * u_{s j}\right\rangle \\
\left\langle p_{g i} * v_{s j}\right\rangle & \left\langle\epsilon_{g i} * v_{s j}\right\rangle & \left\langle u_{g i} * v_{s j}\right\rangle & \left\langle v_{g i} * v_{s j}\right\rangle & \left\langle u_{s i} * v_{s j}\right\rangle & \left\langle v_{s i} * v_{s j}\right\rangle
\end{array}\right)
$$


with $i, j=1, \ldots, N$. The notation $\left\langle\aleph_{i} * \aleph_{j}\right\rangle$ is defined as

$$
\left\langle\aleph_{i} * \aleph_{j}\right\rangle=\frac{1}{M} \sum_{k=1}^{M} \aleph\left(x_{i}, t_{k}\right) \aleph^{T}\left(x_{j}, t_{k}\right)
$$

where $\aleph$ is any field variable, $N$ is the number of nodes and $M$ is the number of snapshots.

In the split approach a separate $\overline{\bar{R}}$ was computed for each field variable. This is analogous to computing a single $\overline{\bar{R}}$ with the off-diagonal sub-matrices set to zero, or

$$
\{\overline{\bar{R}}\}=\left(\begin{array}{cccccc}
\left\{\overline{\bar{R}}_{\left\langle p_{g} * p_{g}\right\rangle}\right\} & 0 & 0 & 0 & 0 & 0 \\
0 & \left\{\overline{\bar{R}}_{\left\langle\epsilon_{g} * \epsilon_{g}\right\rangle}\right\} & 0 & 0 & 0 & 0 \\
0 & 0 & \left\{\overline{\bar{R}}_{\left\langle u_{g} * u_{g}\right\rangle}\right\} & 0 & 0 & 0 \\
0 & 0 & 0 & \left\{\overline{\bar{R}}_{\left\langle v_{g} * v_{g}\right\rangle}\right\} & 0 & 0 \\
0 & 0 & 0 & 0 & \left\{\overline{\bar{R}}_{\left\langle u_{s} * u_{s}\right\rangle}\right\} & 0 \\
0 & 0 & 0 & 0 & 0 & \left\{\overline{\bar{R}}_{\left\langle v_{s} * v_{s}\right\rangle}\right\}
\end{array}\right) .
$$

In practice, the eigenvectors of these sub-matrices were computed separately for the split approach and the method of snapshots (Sirovich, 1987) was used to reduce the size of the matrices for both the coupled and the split approach. This method reduces the dimension of the autocorrelation matrix from the number of nodes to the number of snapshots, greatly speeding computation.

Two sets of basis functions were calculated using the two proposed $\overline{\bar{R}}$ matrices. The solutions were then reconstructed using Equation (18), where the time coefficients were calculated using the projection method,

$$
\alpha_{k}\left(t_{i}\right)=\left(u\left(x, t_{i}\right), \varphi(x)\right), \quad i=1, \ldots, M \text {. }
$$

The errors for each approach, as given by Equation (39), were then compared to determine the better method.

The errors computed using Equation (39) for the two approaches are given in Table 18 using the mode set specified in Table 17. Note that the coupled approach required the field variables to be of the same order of magnitude to produce an autocorrelation matrix with eigenvectors that could be calculated. To accomplish this, each variable was divided by its maximum value prior to the concatenation step. Once the POD was carried out and the solution was reconstructed, each variable was multiplied by the original scaling factor. Then the error was computed to give the values in Table 18 .

As Table 18 shows, the POD reconstruction error was larger for the coupled approach than for the split approach for each variable - sometimes significantly so. For the gas and solids velocities the difference in error between the two methods is only about an order of magnitude. Both the gas pressure and void fraction, however, show a five order of magnitude difference in their error. 
Table 18: POD reconstruction error using Equation (39) for coupled and split approaches and the relative value of these errors, defined by the error divided by the maximum value of the variable.

\begin{tabular}{lcccc}
\hline Variable & \multicolumn{4}{c}{ Error } \\
& $\varepsilon^{\text {coupled }}$ & $\varepsilon^{\text {split }}$ & $\varepsilon_{\text {rel }}^{\text {coupled }}$ & $\varepsilon_{\text {rel }}^{\text {split }}$ \\
\hline$p_{g}$ & $1.245 \mathrm{E}+006$ & 57.91 & 1.230 & $5.72 \mathrm{E}-007$ \\
$\epsilon_{g}$ & $1.012 \mathrm{E}-005$ & $5.891 \mathrm{E}-010$ & $1.012 \mathrm{E}-005$ & $5.891 \mathrm{E}-010$ \\
$u_{g}$ & $1.305 \mathrm{E}-002$ & $5.081 \mathrm{E}-003$ & $9.758 \mathrm{E}-004$ & $3.799 \mathrm{E}-004$ \\
$v_{g}$ & 1.380 & $8.980 \mathrm{E}-003$ & $8.944 \mathrm{E}-002$ & $5.820 \mathrm{E}-004$ \\
$u_{s}$ & $4.251 \mathrm{E}-005$ & $4.833 \mathrm{E}-006$ & $3.271 \mathrm{E}-004$ & $3.719 \mathrm{E}-005$ \\
$v_{s}$ & $2.248 \mathrm{E}-004$ & $4.866 \mathrm{E}-005$ & $1.613 \mathrm{E}-003$ & $3.491 \mathrm{E}-004$ \\
\hline
\end{tabular}

In the case of the void fraction this difference is not very significant. The void fraction can be expressed as

$$
\epsilon_{g}\left(x, t_{i}\right)=\varphi_{0}^{\epsilon_{g}}(x)+\sum_{k=1}^{M} \alpha_{k}^{\epsilon_{g}}\left(t_{i}\right) \varphi_{k}^{\epsilon_{g}}(x), \quad i=1, \ldots, M .
$$

The $\varphi_{0}^{\epsilon_{g}}(x)$ term is the time-average of the solution and is identical for both approaches. This time-average is always positive and less than one. The error calculated for the coupled approach is five orders of magnitude smaller than this average and the error for the split approach was ten orders of magnitude less. Both approaches allow the model to compute the void fraction dynamics by any reasonable measure.

For the gas pressure, however, the difference in error is significant. Recall the POD approximation for $p_{g}$ :

$$
p_{g}\left(x, t_{i}\right)=\varphi_{0}^{p_{g}}(x)+\sum_{k=1}^{M} \alpha_{k}^{p_{g}}\left(t_{i}\right) \varphi_{k}^{p_{g}}(x), \quad i=1, \ldots, M
$$

The error in the coupled approach is on the same order as $\varphi_{0}^{p_{g}}$, which is on the order of $10^{6}$. The difference in error between the two approaches is therefore quite significant.

Based on the POD errors calculated using both a coupled and a split approach to computing the autocorrelation matrix and given by Table 18, the split approach is clearly superior for the test case explored. The best method for coupling the field variables in POD is an ongoing topic of study.

\subsubsection{Augmented Proper Orthogonal Decomposition}

Flows with moving discontinuities cause the current POD approximation to produce poor results. In the usual POD method, the time average is subtracted from the data prior to 
computing the POD modes. This average mode captures any stationary discontinuities in the data. Moving discontinuities, however, are approximated as the superposition of modes of increasing frequency. This approximation is susceptible to dispersion errors that result in the well known Gibbs phenomenon of non-physical oscillations about the discontinuity.

Two common examples of moving discontinuities in flows that are challenging for PODbased ROMs are: (i) moving shocks and (ii) gas bubbles in multiphase flow. For flows with moving shocks, a solution of domain decomposition was proposed where the full-order model (FOM) was used in regions where a shock is possible and a ROM was used everywhere else (Lucia et al. 2003).

In multiphase flow, gas bubbles represent moving discontinuities and the resulting oscillations can cause non-physical values for the void fraction. These spurious values for the void fraction can then cascade through the other field variables and lead to divergence of the computation. In multiphase flow, bubbles can exist in most of the physical domain, rendering the domain decomposition approach impractical. The work presented herein seeks to augment the POD bases with non-orthogonal modes that capture the moving discontinuities exactly and prevent the Gibbs phenomenon from occurring.

The simplest model for a moving discontinuity is the first-order wave equation

$$
\frac{\partial u}{\partial t}+c \frac{\partial u}{\partial x}=0
$$

The wave equation models an initial velocity distribution that propagates with velocity $c$. This model was selected for the ease of predicting the location of the discontinuity, which simplifies the calculation of the discontinuity modes used to augment the POD basis functions in the ROM. At a given time $t$, the discontinuity location, $x_{s}$, is given by

$$
x_{s}=c t+x_{s, 0}
$$

where $x_{s, 0}$ is the initial location of the discontinuity.

The first-order wave equation has a number of exact solutions, but for this problem a simple quadratic polynomial solution given by

$$
u(x, t)=a+b(x-c t)+d(x-c t)^{2}
$$

was used. A discontinuity was added to the initial condition at time $t=0$ through superposition according to

$$
u(x, t=0)= \begin{cases}a+b x+d x^{2}+1 & x \leq x_{s, 0} . \\ a+b x+d x^{2} & x>x_{s, 0} .\end{cases}
$$

Using a first-order forward difference in time and a first-order backward difference in 
space, Equation (43) was discretized as

$$
\frac{u_{i}^{n+1}-u_{i}^{n}}{\Delta t}+c \frac{u_{i}^{n}-u_{i-1}^{n}}{\Delta x}=0
$$

where $u_{i}^{n+1}:=u\left(x_{i}, t^{n+1}\right), u_{i}^{n}:=u\left(x_{i}, t^{n}\right), u_{i-1}^{n}:=u\left(x_{i-1}, t^{n}\right)$, and $\Delta x$ and $\Delta t$ were constant over the integration interval.

Using Equation (14), the solution, $u_{i}^{n+1}$, was then computed over the desired time interval and a snapshot was collected at every time step. The time step size was computed using the CFL condition such that

$$
\Delta t \leq \frac{\Delta x}{c}
$$

Here, $\Delta x$ was determined by evenly spacing 100 grid points over the interval $0 \leq x \leq 1$. The propagation speed was $c=0.5$. One time unit was simulated, resulting in the collection of 50 snapshots.

Recall that in Equation (18), with the average mode $\varphi_{0}$, a function $u(\mathbf{x}, t)$ can be approximated as,

$$
u\left(\mathbf{x}, t_{k}\right)=\varphi_{0}(\mathbf{x})+\sum_{j=1}^{m} \alpha_{j}\left(t_{k}\right) \varphi_{j}(\mathbf{x})
$$

A series of discontinuity modes, denoted by $\psi$, can be added to the approximation so that

$$
u\left(\mathbf{x}, t_{k}\right)=\varphi_{0}(\mathbf{x})+\sum_{j=1}^{m} \alpha_{j}\left(t_{k}\right) \varphi_{j}(\mathbf{x})+\sum_{\ell=1}^{m_{a}} \beta_{\ell}\left(t_{k}\right) \psi_{\ell}\left(\mathbf{x}, t_{k}\right) \beta_{\ell}\left(t_{k}\right),
$$

where $\beta_{\ell}$ are the time coefficients associated with the discontinuity modes $\psi_{\ell}$. Substituting this approximation into Equation (43) gives

$$
\begin{aligned}
\frac{\partial}{\partial t}\left(\sum_{j=0}^{m} \varphi_{j}(\mathbf{x}) \alpha_{j}\left(t_{k}\right)+\sum_{\ell=1}^{m_{a}} \psi_{\ell}\left(\mathbf{x}, t_{k}\right)\right. & \left.\beta_{\ell}\left(t_{k}\right)\right) \\
& +c \frac{\partial}{\partial x}\left(\sum_{j=0}^{m} \varphi_{j}(\mathbf{x}) \alpha_{j}\left(t_{k}\right)+\sum_{\ell=1}^{m_{a}} \psi_{\ell}\left(\mathbf{x}, t_{k}\right) \beta_{\ell}\left(t_{k}\right)\right)=0
\end{aligned}
$$


or

$$
\begin{aligned}
\sum_{j=1}^{m} \dot{\alpha}_{j}\left(t_{k}\right) \varphi_{j}(\mathbf{x})+\sum_{\ell=1}^{m_{a}} \dot{\beta}_{\ell}\left(t_{k}\right) \psi_{\ell}\left(\mathbf{x}, t_{k}\right) & +\sum_{\ell=1}^{m_{a}} \beta_{\ell}\left(t_{k}\right) \dot{\psi}_{\ell}\left(\mathbf{x}, t_{k}\right) \\
& +c \sum_{j=0}^{m} \alpha_{j}\left(t_{k}\right) \varphi_{j}^{\prime}(\mathbf{x})+\sum_{\ell=1}^{m_{a}} \beta_{\ell}\left(t_{k}\right) \psi_{\ell}^{\prime}\left(\mathbf{x}, t_{k}\right)=0
\end{aligned}
$$

where the notation $\dot{u}:=\frac{\partial u}{\partial t}$ and $u^{\prime}:=\frac{\partial u}{\partial x}$ is used for derivatives. Projecting Equation (45) onto the augmented set of basis functions using a Galerkin projection, a system of equations is obtained given by

$$
[A]\left\{\begin{array}{c}
\dot{\alpha} \\
\dot{\beta}
\end{array}\right\}+[B]\left\{\begin{array}{c}
\alpha \\
\beta
\end{array}\right\}+\{d\}=\{0\},
$$

with $[A] \in \mathbb{R}^{m+m_{a}} \times \mathbb{R}^{m+m_{a}},\{\alpha\} \in \mathbb{R}^{m},\{\beta\} \in \mathbb{R}^{m_{a}}$ and $\{d\} \in \mathbb{R}^{m+m_{a}}$. The elements of the $[A]$ and $[B]$ matrices and the $\{d\}$ vector are

$$
\begin{gathered}
A_{i j}=\left\{\begin{array}{lll}
\left(\varphi_{j}, \varphi_{i}\right)=\delta_{j i} & i=1, \ldots, m & j=1, \ldots, m \\
\left(\varphi_{j}, \psi_{i}\right) & i=m+1, \ldots, m+m_{a} & j=1, \ldots, m \\
\left(\psi_{j}, \varphi_{i}\right) & i=1, \ldots, m & j=m+1, \ldots, m+m_{a} \\
\left(\psi_{j}, \psi_{i}\right) & i=m+1, \ldots, m+m_{a} & j=m+1, \ldots, m+m_{a}
\end{array}\right. \\
B_{i j}=\left\{\begin{array}{lll}
c\left(\varphi_{j}^{\prime}, \varphi_{i}\right) & i=1, \ldots, m & j=1, \ldots, m \\
c\left(\varphi_{j}^{\prime}, \psi_{i}\right) & i=m+1, \ldots, m+m_{a} & j=1, \ldots, m \\
\left(\dot{\psi}_{j}+c \psi_{j}^{\prime}, \varphi_{i}\right) & i=1, \ldots, m & j=m+1, \ldots, m+m_{a} \\
\left(\dot{\psi}_{j}+c \psi_{j}^{\prime}, \psi_{i}\right) & i=m+1, \ldots, m+m_{a} & j=m+1, \ldots, m+m_{a}
\end{array}\right.
\end{gathered}
$$

and

$$
d_{i}= \begin{cases}c\left(\varphi_{0}, \varphi_{i}\right) & i=1, \ldots, m \\ c\left(\varphi_{0}, \psi_{i}\right) & i=m+1, \ldots, m+m_{a}\end{cases}
$$

Note that the discontinuity modes, $\psi_{\ell}$, are defined as having unit value on one side of a discontinuity and are zero on the other side. The modes are therefore discontinuous and the spatial derivative, $\psi_{\ell}^{\prime}$, is the Dirac delta function, $\delta(x)$, centered at the shock location. Then for $j=m+1, \ldots, m+m_{a}$, by definition

$$
\begin{array}{ll}
\left(c \psi_{j}^{\prime}, \varphi_{i}\right)=\left(c \cdot \delta\left(x_{s_{j}}\right), \varphi_{i}\right)=c \varphi_{i}\left(x_{s_{j}}\right) & i=1, \ldots, m \\
\left(c \psi_{j}^{\prime}, \psi_{i}\right)=\left(c \cdot \delta\left(x_{s_{j}}\right), \psi_{i}\right)=c \psi_{i}\left(x_{s_{j}}\right) & i=m+1, \ldots, m+m_{a}
\end{array}
$$


where $x_{s_{j}}$ is the location of the discontinuity in the $j$ th discontinuity mode. Therefore,

$$
B_{i j}=\left\{\begin{array}{lll}
c\left(\varphi_{j}^{\prime}, \varphi_{i}\right) & i=1, \ldots, m & j=1, \ldots, m \\
c\left(\varphi_{j}^{\prime}, \psi_{i}\right) & i=m+1, \ldots, m+m_{a} & j=1, \ldots, m \\
\left(\dot{\psi}_{j}, \varphi_{i}\right)+c \varphi_{i}\left(x_{s_{j}}\right) & i=1, \ldots, m & j=m+1, \ldots, m+m_{a} \\
\left(\dot{\psi}_{j}, \psi_{i}\right)+c \psi_{i}^{\prime}\left(x_{s_{j}}\right) & i=m+1, \ldots, m+m_{a} & j=m+1, \ldots, m+m_{a}
\end{array}\right.
$$

Note that for the simple test case considered, only one discontinuity mode was used, i.e., $m_{a}=1$. An adaptive Runge-Kutta-Fehlberg method (Fehlberg, 1969) was then used to solve the system of ODEs given by Equation (46).

This method has been labeled PODA, with the A standing for analytical. In this approach, the POD approximation is substituted directly into the governing equation and the temporal and spatial discretizations are not performed in the derivation. This allows flexibility in the discretization, depending on the specific problem encountered. A second method was considered, labeled PODD, where the D is for discretized. In the PODD approach the discretizations are performed prior to the substitution of the POD approximation into the governing equation. This approach is considered next.

Keeping the convention that $n$ gives the current time step, $i$ the current spatial location, and $j$ and $\ell$ the mode, the terms in the right hand side of Equation (44) can be approximated by

$$
\begin{aligned}
& u_{i}^{n+1}=\varphi_{0_{i}}+\sum_{j=1}^{m} \alpha_{j}^{n+1} \varphi_{j_{i}}+\sum_{\ell=1}^{m_{a}} \beta_{\ell}^{n+1} \psi_{\ell_{i}}^{n+1} \\
& u_{i}^{n}=\varphi_{0_{i}}+\sum_{j=1}^{m} \alpha_{j}^{n} \varphi_{j_{i}}+\sum_{\ell=1}^{m_{a}} \beta_{\ell}^{n} \psi_{\ell_{i}}^{n} \\
& u_{i-1}^{n}=\varphi_{0_{i-1}}+\sum_{j=1}^{m} \alpha_{j}^{n} \varphi_{j_{i-1}}+\sum_{\ell=1}^{m_{a}} \beta_{\ell}^{n} \psi_{\ell_{i-1}}^{n} .
\end{aligned}
$$

Substituting equations (47)-(49) into Equation (44) yields

$$
\begin{aligned}
\sum_{j=0}^{m} \alpha_{j}^{n+1} \varphi_{j_{i}}+\sum_{\ell=1}^{m_{a}} \beta_{\ell}^{n+1} \psi_{\ell_{i}}^{n+1} & =\sum_{j=0}^{m} \alpha_{j}^{n} \varphi_{j_{i}}+\sum_{\ell=1}^{m_{a}} \beta_{\ell}^{n} \psi_{\ell_{i}}^{n} \\
& -\frac{c \Delta t}{\Delta x}\left(\sum_{j=0}^{m} \alpha_{j}^{n} \varphi_{j_{i}}+\sum_{\ell=1}^{m_{a}} \beta_{\ell}^{n} \psi_{\ell_{i}}^{n}-\sum_{j=0}^{m} \alpha_{j}^{n} \varphi_{j_{i-1}}+\sum_{\ell=1}^{m_{a}} \beta_{\ell}^{n} \psi_{\ell_{i-1}}^{n}\right) .
\end{aligned}
$$

This form of the governing equation can then be projected onto the basis functions, 
$\left\{\varphi_{k}, \psi_{k}\right\}$ to obtain a system of ordinary differential equations as an initial value problem with the time coefficients, $\left\{\alpha_{j}, \beta_{j}\right\}$, as the unknowns. The method is explicit, so the time coefficients at the $n$-th step are known and the field variable can be reconstructed to find $u_{i}^{n}$. The Galerkin projection of the governing equation is

$$
\begin{aligned}
& \left(\sum_{j=0}^{m} \alpha_{j}^{n+1} \varphi_{j_{i}}+\sum_{\ell=1}^{m_{a}} \beta_{\ell}^{n+1} \psi_{\ell}^{n+1}, \varphi_{k_{i}}\right) \\
& =\left(u_{i}^{n}-\frac{c \Delta t}{\Delta x}\left(u_{i}^{n}-u_{i-1}^{n}\right), \varphi_{k_{i}}\right) \quad k=1, \ldots, m \\
& \left(\sum_{j=0}^{m} \alpha_{j}^{n+1} \varphi_{j_{i}}+\sum_{\ell=1}^{m_{a}} \beta_{\ell}^{n+1} \psi_{\ell}^{n+1}, \psi_{k_{i}}^{n}\right) \\
& =\left(u_{i}^{n}-\frac{c \Delta t}{\Delta x}\left(u_{i}^{n}-u_{i-1}^{n}\right), \psi_{k_{i}}^{n}\right) \quad k=m+1, \ldots, m+m_{a}
\end{aligned}
$$

The contribution of the average mode, $\varphi_{0}$ on the $u_{i}^{n+1}$ term is moved to the right hand side so that

$$
\left[A_{D}\right]\left\{\begin{array}{c}
\alpha^{n+1} \\
\beta^{n+1}
\end{array}\right\}=\left\{b_{D}\right\}
$$

where

$$
A_{D j k}=\left\{\begin{array}{lll}
\left(\varphi_{k_{i}}, \varphi_{j_{i}}\right)=\delta_{k j} & j=1, \ldots, m & k=1, \ldots, m \\
\left(\varphi_{k_{i}}, \psi_{j_{i}}^{n+1}\right) & j=m+1, \ldots, m+m_{a} & k=1, \ldots, m \\
\left(\psi_{k_{i}}^{n+1}, \varphi_{j_{i}}\right) & j=1, \ldots, m & k=m+1, \ldots, m+m_{a} \\
\left(\psi_{k_{i}}^{n+1}, \psi_{j_{i}}^{n+1}\right) & j=m+1, \ldots, m+m_{a} & k=m+1, \ldots, m+m_{a}
\end{array}\right.
$$

and

$$
b_{D k}=\left\{\begin{array}{l}
\left(u_{i}^{n}-\frac{c \Delta t}{\Delta x}\left(u_{i}^{n}-u_{i-1}^{n}\right), \varphi_{k_{i}}\right. \\
\left(u_{i}^{n}-\frac{c \Delta t}{\Delta x}\left(u_{i}^{n}-u_{i-1}^{n}\right), \psi_{k_{i}}^{n}\right.
\end{array}\right)-\left(\begin{array}{l}
\varphi_{0_{i}}, \varphi_{k_{i}} \\
\varphi_{0_{i}}, \psi_{k_{i}}^{n}
\end{array}\right) \begin{aligned}
& k=1, \ldots, m \\
& k=m+1, \ldots, m+m_{a}
\end{aligned}
$$

Here $j$ and $k$ denote the modes, $i$ is the spatial location and $n$ is the time step. Note that $\psi$ is a function of time and it is therefore necessary to consider both $\psi^{n}$ and $\psi^{n+1}$. A conjugate gradient method was used to solve this system of equations.

A set of time coefficients were calculated directly by projecting the snapshots onto the 
basis functions (Cizmas and Palacios, 2003). This gave the best case solution for the flow at reference conditions. The POD approximation

$$
u_{a}\left(\mathbf{x}, t_{k}\right)=\sum_{j=0}^{m} \alpha_{j}\left(t_{k}\right) \varphi_{j}(\mathbf{x})+\sum_{\ell=1}^{m_{a}} \beta_{\ell}\left(t_{k}\right) \psi_{\ell}\left(\mathbf{x}, t_{k}\right)
$$

was then projected onto the augmented set of basis functions using a Galerkin projection such that

$$
\begin{aligned}
& \left(u_{a}-\varphi_{0}, \varphi_{i}\right)=\left(\sum_{j=1}^{m} \alpha_{j} \varphi_{j}+\sum_{\ell=1}^{m_{a}} \beta_{\ell} \psi_{\ell}, \varphi_{i}\right) \quad i=1, \ldots, m \\
& \left(u_{a}-\varphi_{0}, \psi_{i}\right)=\left(\sum_{j=1}^{m} \alpha_{j} \varphi_{j}+\sum_{\ell=1}^{m_{a}} \beta_{\ell} \psi_{\ell}, \psi_{i}\right) \quad i=m+1, \ldots, m+m_{a}
\end{aligned}
$$

It is possible for the discontinuity modes to not be orthogonal to the POD bases. As a result the system does not decouple and the time coefficients are found by solving the system

$$
\left[A_{E}\right]\left\{\begin{array}{l}
\alpha \\
\beta
\end{array}\right\}=\left\{b_{E}\right\}
$$

where, using $i$ and $j$ to denote the modes,

$$
A_{E i j}=\left\{\begin{array}{lll}
\left(\varphi_{j}, \varphi_{i}\right)=\delta_{j i} & i=1, \ldots, m & j=1, \ldots, m \\
\left(\varphi_{j}, \psi_{i}\right) & i=m+1, \ldots, m+m_{a} & j=1, \ldots, m \\
\left(\psi_{j}, \varphi_{i}\right) & i=1, \ldots, m & j=m+1, \ldots, m+m_{a} \\
\left(\psi_{j}, \psi_{i}\right) & i=m+1, \ldots, m+m_{a} & j=m+1, \ldots, m+m_{a}
\end{array}\right.
$$

and

$$
b_{E i}= \begin{cases}\left(u_{a}-\varphi_{0}, \varphi_{i}\right) & i=1, \ldots, m \\ \left(u_{a}-\varphi_{0}, \psi_{i}\right) & i=m+1, \ldots, m_{a}\end{cases}
$$

Up to this point, very little has been said about the structure of the discontinuity modes. Note that the POD basis functions do not span the entire space because the set of basis functions was truncated and the highest-order modes discarded. The discontinuity modes must be linearly independent of the POD basis functions and it was therefore necessary to test the augmented set of basis functions to check whether the discontinuity modes, $\{\psi\}$ could be expressed as a linear combination of $\varphi_{i}, i \in[0, m]$.

To ensure this linear independence, the discontinuity modes were appended to the matrix of basis functions at every time step and the singular value decomposition was computed. If the smallest singular value of this matrix was less than an error tolerance, the corresponding discontinuity mode was not linearly independent and was therefore not needed in the calculation.

For simplicity, herein each discontinuity mode was a simple step function with a value of one upstream of the discontinuity and zero downstream of the discontinuity. For the 
simple test case considered in this paper, only one discontinuity was present at any time and its location was always known, so only one discontinuity mode was needed. In practice, a number of discontinuity modes could have been used, each representing a possible location for the discontinuity. Since each discontinuity mode in this collection was constant in time, $\dot{\psi}_{\ell}$ was assumed to be zero.

The velocity profile for the first-order wave equation case is shown in Figure 32 at $t=1.0$ for the FOM, the non-augmented reduced-order model and the augmented reduced-order model. The non-augmented ROM used 20 modes while the augmented ROM used only two modes. The non-augmented ROM shows the Gibbs phenomenon while the augmented ROM does not have oscillations present.

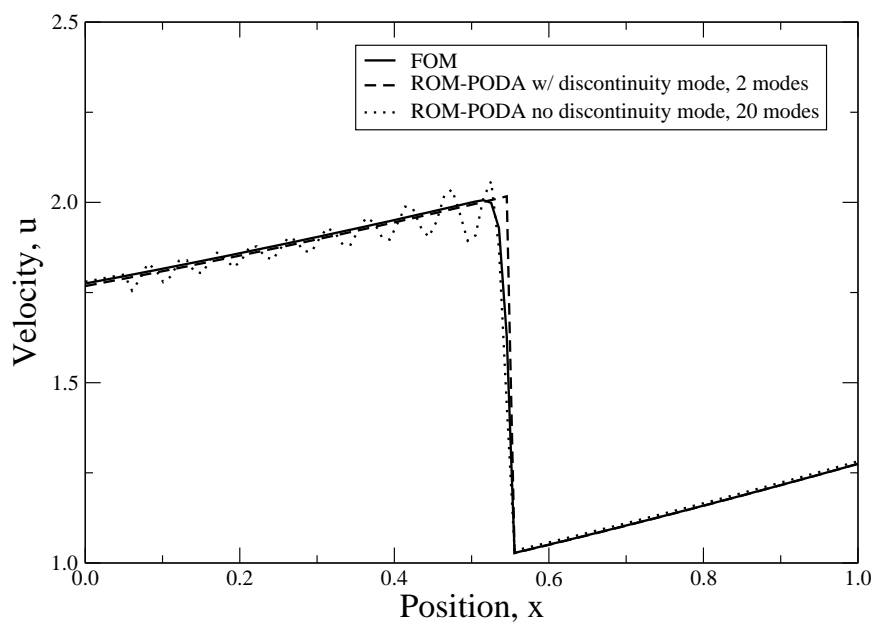

Figure 32: Velocity profile at $t=1.0 \mathrm{~s}$ for full-order model, reduced-order model with 20 modes and no discontinuity mode, and reduced-order model with 2 modes, including a discontinuity mode.

Figures 33 and 34 show the first two time coefficients, respectively, computed using the ROM with a discontinuity mode, compared to the results obtained by projecting the snapshots directly onto the basis functions. Note that for this problem, the second mode time coefficient is the discontinuity mode time coefficient.

The computed time coefficients show excellent agreement with the reference solution. This is further reinforced by the lack of Gibbs oscillations in the solution of the augmented POD method. The additional computations created by the non-orthogonality of the modes are more than accounted for by the reduction in modes from 20 to 2 when the augmented ROM is compared to the traditional ROM. As an additional benefit, the solution computed using the augmented ROM is not degraded by numerical dissipation around the discontinuity as the FOM is. 


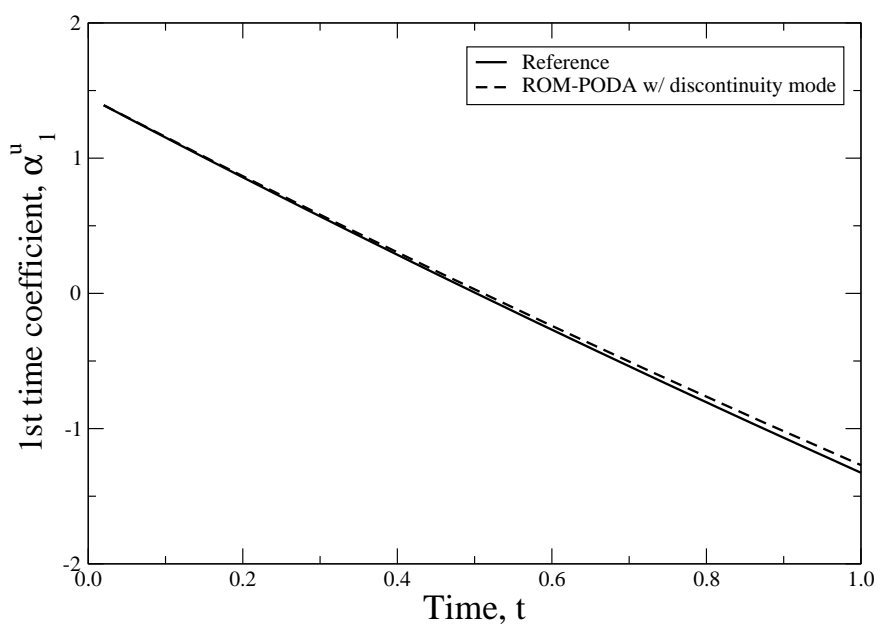

Figure 33: First mode time coefficient computed using the augmented ROM and compared to a reference computed by projecting the snapshots onto the basis functions.

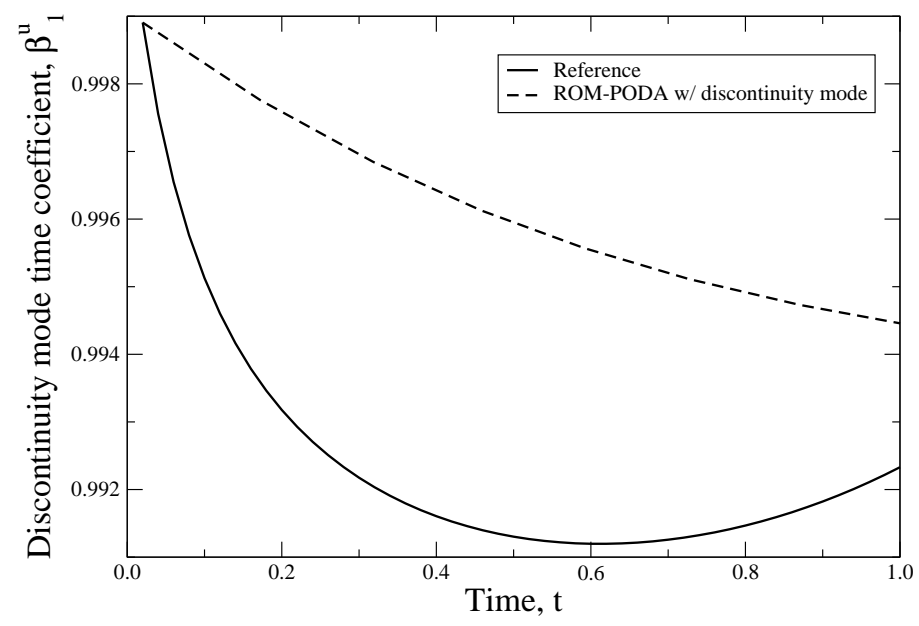

Figure 34: Time coefficient for discontinuity mode computed using the augmented ROM and compared to a reference computed by projecting the snapshots onto the basis functions. 
This work has demonstrated that for a very simple test case, it is possible to augment the POD bases with a non-orthogonal discontinuity mode that travels in both space and time. This augmented collection of basis functions produces a more accurate solution with considerably fewer modes and demonstrates no Gibbs oscillations. The non-orthogonality of the modes causes an increase in the number of computations required per mode for each time step.

Two approaches to formulating reduced-order models were presented. The two approaches are computationally identical when the same spatial and temporal discretizations were used. The PODA formulation was deemed to be more useful, as it allows the specification of an appropriate accuracy on a problem by problem basis.

\subsubsection{Point-Mode POD}

Another method for adapting the POD method to solve for moving discontinuities is the point-mode method. In this approach, a collection of point modes are added to the set of POD basis functions. Point modes are defined as having a value of one for a single cell in the computational mesh and are zero everywhere else. If the POD basis functions are then set equal to zero in the cells for which point modes are used, the computational domain will be automatically decomposed into areas where the ROM is solved and areas where the FOM is solved.

To see this, consider the discretized $x$-direction conservation of momentum equation:

$$
a_{p}\left(u_{g}\right)_{p}+\sum_{n b} a_{n b}\left(u_{g}\right)_{n b}=b_{p}
$$

The $x$-direction gas velocity can be approximated as

$$
u_{g}(x, t)=\phi_{0}(x)+\sum_{j=1}^{n} \phi_{j}(x) \alpha_{j}(t)+\sum_{j=n+1}^{n+m} \phi_{j}^{p m}(x) \alpha_{j}^{p m}(t),
$$

where $\phi_{j}$ represents a POD basis function and $\phi_{j}^{p m}$ is a point mode. The collection of POD and point modes is then defined as $\{\psi\}$ where

$$
\psi_{j}= \begin{cases}\phi_{j} & 0 \leq j \leq n \\ \phi_{j}^{p} & n+1 \leq j \leq n+m\end{cases}
$$

Note that the POD basis functions that are included in this collection of modes have been masked, that is set to zero, at the spatial points where point modes exist. Substituting Equation (51) into Equation (50), using a Galerkin projection to project the resulting equation onto $\{\psi\}$ and rearranging, the system of equations to be solved for the time coefficients can 
be expressed as

$$
A_{i j} \alpha_{j}=b_{i}
$$

Here

$$
A_{i j}=\left(a_{p}\left(\psi_{j}\right)_{p}+\sum_{n b} a_{n b}\left(\psi_{j}\right)_{n b}, \psi_{i}\right)
$$

and

$$
b_{i}=\left(b_{p}-\sum_{n b} a_{n b}\left(\psi_{0}\right)_{n b}-a_{p}\left(\psi_{0}\right)_{p}, \psi_{i}\right)
$$

Consider a point mode, $\phi_{j}^{p m}$ defined at a point $x_{p}$. This point mode will have a time coefficient, $\alpha_{j}^{p m}$. The point mode is orthogonal to every other mode in the set $\{\psi\}$ so the equation for $\alpha_{j}^{p m}$ is

$$
a_{p}^{x_{p}} \alpha^{p}+\sum_{n b} a_{n b}^{x_{p}} \sum_{j=0}^{n+m}\left(\psi_{j}\right)_{n b} \alpha_{j}=b_{p}^{x_{p}}
$$

where $a_{p}^{x_{p}}, a_{n b}^{x_{p}}$ and $b_{p}^{x_{p}}$ are the coefficients from the discretized momentum equation for the point $x_{p}$. Recognizing that

$$
\left(u_{g}\right)_{n b} \approx \sum_{j=0}^{n+m}\left(\psi_{j}\right)_{n b} \alpha_{j}
$$

regardless of whether the neighbor cell is a point-mode cell or a POD mode cell, then

$$
a_{p}^{x_{p}} \alpha^{p}+\sum_{n b} a_{n b}^{x_{p}}\left(u_{g}\right)_{n b}=b_{p}^{x_{p}}
$$

Clearly $\alpha^{p}\left(t_{k}\right)$ is an approximation for $u_{g}\left(x_{p}, t_{k}\right)$.

To demonstrate this, 12 point modes were defined for $u_{g}$ and used in a test case whose parameters are given in Table 2. The geometry for this case is shown in Fig. 1. The point modes were placed in a block, just above the jet inlet. A zoomed view of the average mode, $\phi_{0}$, is shown in Fig. 35 both before and after masking. Figure 36 indicates the location of two of the point modes. Finally, Fig. 37 compares the time history of the $x$-direction gas velocity computed with MFIX at these two points to the time history of the time coefficients associated with the two point modes. Good agreement is observed, considering the relatively small variation in the velocity in this simple test case.

The logical next step is to apply this method to the ROM for void fraction. Unfortunately, as ODEx is currently written, the time coefficient associated with a point mode would 


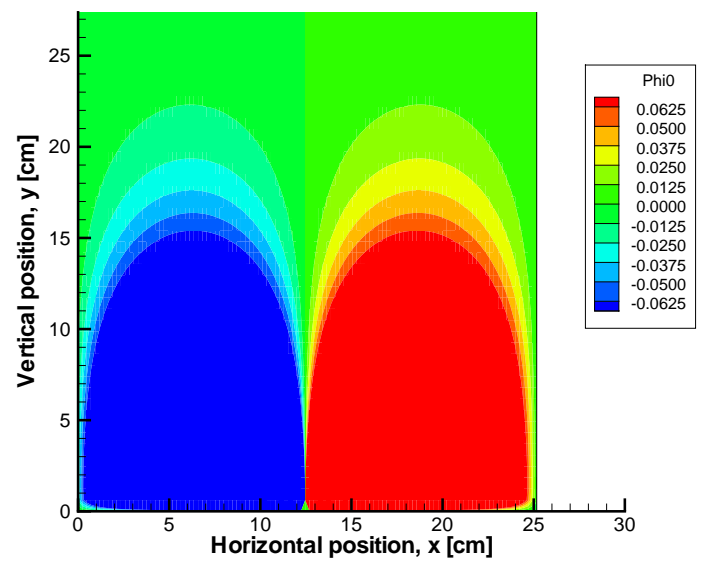

(a)

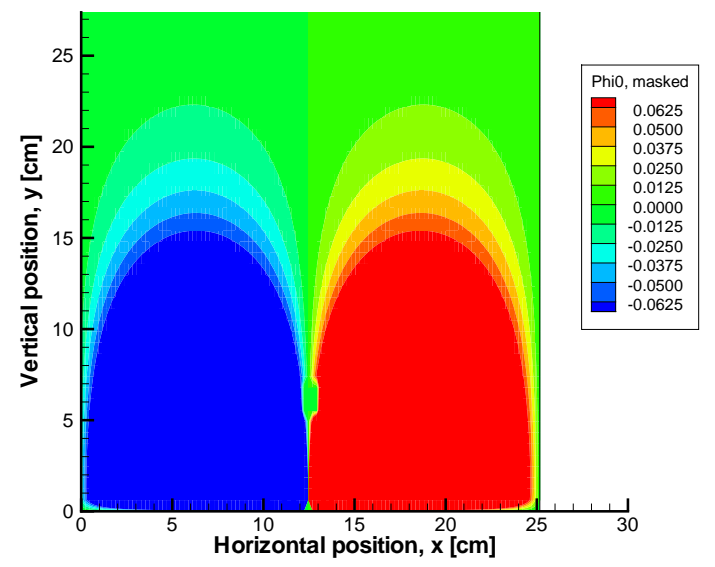

(b)

Figure 35: Contour plots of the average mode for $u_{g}$ : (a) unmasked, and (b) masked.

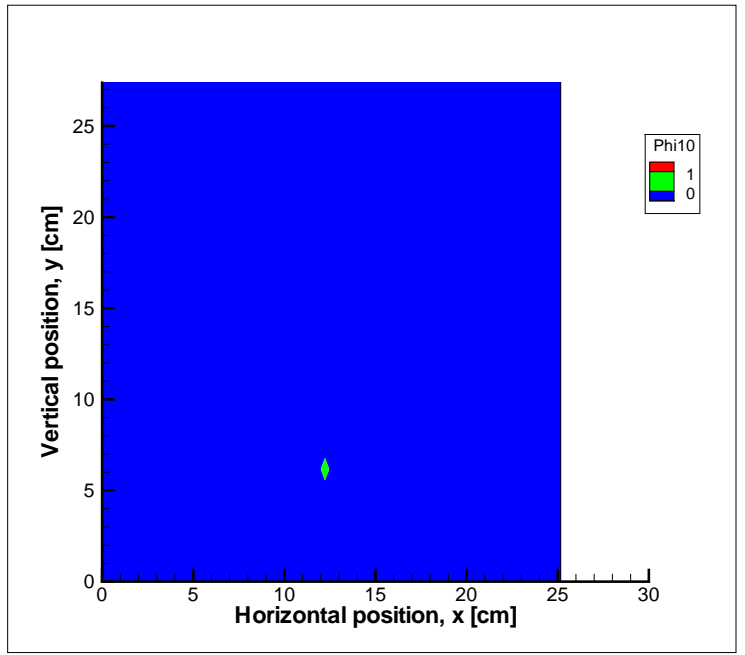

(a)

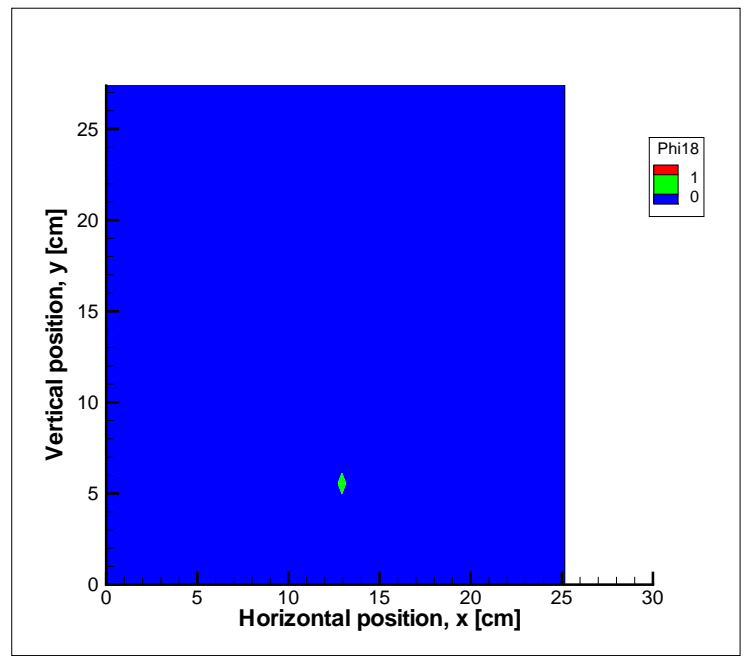

(b)

Figure 36: Contour plots indicating the location of two point modes: (a) $\phi_{10}^{p m}$, and (b) $\phi_{18}^{p m}$. 


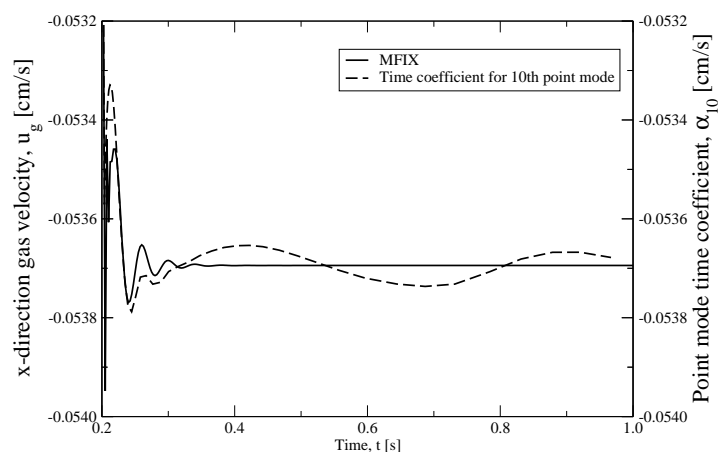

(a)

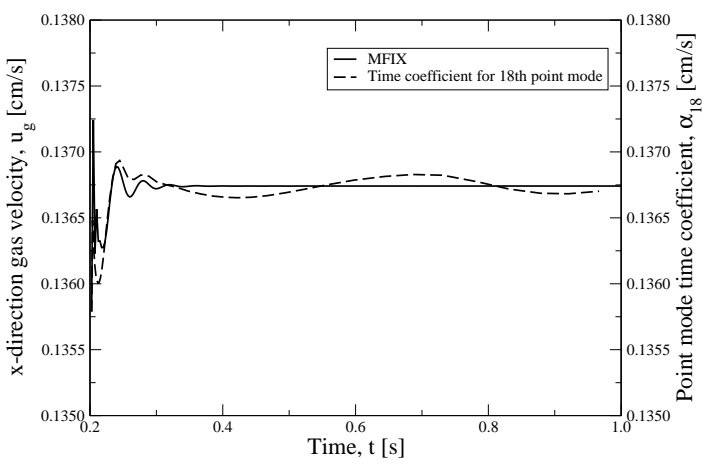

(b)

Figure 37: Time histories for $u_{g}$ at two points in the flow, compared to: (a) $\alpha_{10}^{p m}$, and (b) $\alpha_{18}^{p m}$.

approximate the void fraction correction, while the ROM is solving for the void fraction itself. An additional modification is needed, either reducing an equation that models the void fraction directly, or developing an algorithm that allows the void fraction to be modeled in some region and the void fraction correction in others. This is a possible topic for future development.

\subsubsection{Morphology for Bubble Detection}

No analytical solution exists to determine the location of the moving discontinuities, but they can be located numerically using morphology. Developed in the 1960's by Georges Matheron and Jean Serra at the Ecole des Mines de Paris, France, mathematical morphology is a method for the analysis of spatial structures (5oill, 2003). Morphology is heavily based on set theory, integral geometry, and lattice algebra. Morphology was originally developed to describe porous media, where the porous material can be characterized as a binary image containing pores or solid surrounding material (5oill, 2003). While this method has primarily been used in the image processing community, it also has been used on a wide variety of spatial structures. Herein the theory and application of morphology is extended to locating discontinuities in flows.

While (Gidaspow, 1994, Ch. 6-7) gives some correlations for determining the formation of bubbles as well the Davidson bubble model, which indicates if a bubble is fast or slow, for multiphase flows there are no formulations to predict the number, size, and location of bubbles in a fluidized bed. If POD is to be applied to bubbling fluidized beds using the augmented method discussed herein, a numerical algorithm must be applied to the data on the POD snapshots to determine when the bubbles are generated and where the bubbles are. Using mathematical morphology, these bubbles can be extracted from the data sets as 
the discontinuity modes for later augmentation of the bases.

Morphology attempts to extract structures of interest located within the whole spatial domain, herein referred to as an image, by performing various set operations over so-called structuring elements. Structuring elements are used to probe the image with various shapes that describe the structure of interest. Structuring elements can take on any number of shapes, from simple rods (straight elements) to circles, or in extreme cases, galaxy shapes. Structuring elements can also be applied in combinations to create larger structuring elements of varying shapes. While there is not a limitation to what shapes or size the structuring elements can take, these structuring elements must be aligned in the same direction throughout the image. Failure to maintain the same alignment throughout will distort the structures that are sought. In addition, structuring elements can only be applied to highly structured images. Images not in a highly structured state must be transformed to this state before applying the morphological operations as usual.

The structuring element, denoted as $b$, is a locally defined quantity in the local coordinates $(k, l)$. The values of $(k, l)$ are defined depending on the total size of the structuring element. If $(0,0)$ is the point of interest in local $(k, l)$ coordinates, then for a five point vertical rod structuring element the global coordinates would be $(i, j-2 l),(i, j-l),(i, j),(i, j+l)$, and $(i, j+2 l) . b$ assumes the value of the image, $f$, at these coordinates.

While morphology can perform a wide variety of operations over the structuring elements, all of these operations can be described by combinations of two elementary operations: erosion and dilation. Both of these operations can be described as shifts in the data set. Erosion is the minimum of these shifts of the image, $f(i, j)$, over the structuring element, $b(k, l)$, given as

$$
e(f)=\min (f(i+k, j+l)-b(k, l)) .
$$

Dilation is the maximum of these shifts over the same structuring element,

$$
d(f)=\max (f(i-k, j-l)+b(k, l)) .
$$

Using equations (52) and (53) in conjunction, more powerful operations can be performed. While these operations are not inherently morphological in nature, they are useful for detecting discontinuities in flows. These operations include blurring, blur/erosion, dilation/blur, and thresholding.

Blurring is used in image processing as a noise reduction technique. This operation is required for a preliminary elimination of extreme values. Blurring is also required for locating step-type edges (Lee et a1., 1987). Without blurring, the area around a step edge is not a local maximum or minimum, and the erosion and dilation operations will not extract discontinuities of that type (Lee et al, 1987). Blurring is defined as

$$
\operatorname{blur}(f)=\frac{\sum_{p=i-L}^{i+L} f(p)}{K} \quad L=\frac{K-1}{2} .
$$


Here blurring is defined over the reduced stencil size of nodes, $K$.

While blurring is necessary, using a reduced stencil that is too large will over-blur the image, resulting in overly distorted edges that are impossible to discern. A good reduced stencil size for blurring is $K=3$ (Lee et an., 1987); i.e., when using a vertical rod structuring element and looking at the center point, the values used to blur the center point are, in global coordinates: $(i, j-l),(i, j)$, and $(i, j+l)$. A consequence of this stencil size for blurring is that the structuring elements must be at least two elements larger than the blurring stencil in order to mitigate possible over-distortion of the edges.

The blur/erosion $(b e)$ and dilation/blur $(d b)$ operators were proposed by Lee et al. (Lee et a1., 1987). These operations act over the structuring elements and are used to eliminate obvious points where edges are not located using the standard erosion and dilation operations in conjunction with blurring. These combination operators initially blur the image and then apply the erosion and dilation operators to the blurred image. The be and $d b$ operators are defined as

$$
\operatorname{be}(f)=\operatorname{blur}(f)-e(\operatorname{blur}(f))
$$

and

$$
d b(f)=d(\operatorname{blur}(f))-\operatorname{blur}(f),
$$

respectively.

The last operation required to detect edges is thresholding. Thresholding is an image segmentation technique used to isolate areas of interest in the image. For non-binary images, thresholding is almost always required to completely extract the desired information from the image. Grayscale images can take on a wide variety of values, causing the previously described operations to assign more than two points to an edge, where the edge is defined as being only two points in width; thresholding ensures that each edge is only two points in width. Thresholding can be applied globally or semi-globally, where globally is a binary fix to the image and semi-globally maintains the original value of the edge. We use global thresholding, defined as (Ritter and Wilson, 2000)

$$
\operatorname{thres}(f)= \begin{cases}1 & \alpha \leq f \leq \beta \\ 0 & \text { otherwise }\end{cases}
$$

The constants $\alpha$ and $\beta$ are user defined values specific for the image or set of images if the group of images describe the same general phenomenon.

All of the previously described operations, when combined, allow for the detection of edges in images with two-point edge strength. The algorithm for edge detection, described by Lee, et al. (Lee et al, 1987), is adapted here for multiphase flow.

1. Read in the images with discontinuities to be detected.

2. Create the structuring elements. Herein, we used four rod-type structuring elements 
that are five units in length and have the following orientations: horizontal, vertical, and diagonal (45 and 135 degrees with respect to the horizontal).

3. Blur the image using Equation (54) with $K=3$.

4. Compute the erosion and dilation of the blurred images using equations (52) and (53).

5. Compute values of the blur/erosion and dilation/blur operators with equations (55) and (56).

6. Determine the maximum values of the be and $d b$ operators

$$
\max \left(b e_{i}(f)\right) \quad i=1, \ldots, 4
$$

and

$$
\max \left(d b_{i}(f)\right) \quad i=1, \ldots, 4 \text {. }
$$

where $i$ represents each of the four structuring elements.

7. Determine the edge strength

$$
\text { Indicator }(f)=\min (\max (d b(f)), \max (b e(f))) \text {. }
$$

8. Apply the global thresholding to the images given by Equation (57). This gives edges for the discontinuity that are only two points in width.

The method developed to detect discontinuities was tested using data obtained from the simulation of a bubbling fluidized bed using the Multiphase Flow with Interphase eXchanges (MFIX) code (Syamlal et an, [994). MFIX was developed at the National Energy Technology Laboratory and uses a staggered grid arrangement and a finite volume method (Syamla, 1998). For this case, the conservation of mass and momentum equations were solved. The parameters and geometry for this test case were taken from (Gidaspow, 1994). Figure 1 shows the problem geometry. The flow parameters are given in Table 19 .

Figure 38 shows the effect of reconstructing the void fraction using the POD basis functions obtained from the bubbling case. The time coefficients were calculated by projecting the snapshot at $t=1.0 \mathrm{~s}$ onto the basis functions. The white areas in the flow field are outside of the range of physically possible values, that is, $0.35 \leq \epsilon_{g} \leq 1$. This demonstrates the need for the augmented basis functions.

The results of applying the discontinuity detection algorithm to a snapshot of the void fraction at $t=1.0 \mathrm{~s}$ are given in Figure 39. For this problem, the thresholding constants $\alpha$ and $\beta$ were varied from 0.0 to 0.4 . The values giving the best discontinuity detection were found to be $\alpha=0.1$ and $\beta=0.2$. Note that all of the high energy bubbles, those containing high concentrations of gas, are easily extracted. Lower energy bubbles, however, are not indicated. This arises from the application of the thresholding technique. While the algorithm still detects these lower energy bubbles, thresholding the data set eliminates these bubbles due to their low indication energy, given by Equation (57). Unlike the high-energy 
Table 19: Parameters of fluidized bed Gidaspow (1994).

\begin{tabular}{llcl}
\hline Parameter & Description & Units & \\
\hline$x_{\text {length }}$ & Length of the domain in $x$-direction & $\mathrm{cm}$ & 39.37 \\
$y_{\text {length }}$ & Length of the domain in $y$-direction & $\mathrm{cm}$ & 58.44 \\
$i_{\text {max }}$ & Number of cells in $x$-direction & - & 108 \\
$j_{\text {max }}$ & Number of cells in $y$-direction & - & 124 \\
$v_{1}, v_{2}$ & Gas inflow velocities & $\mathrm{cm} / \mathrm{s}$ & $355.0,28.4$ \\
$p_{g_{s}}$ & Static pressure at outlet & $\mathrm{g} / \mathrm{cm} / \mathrm{s}^{2}$ & $1.01 e^{6}$ \\
$T_{g_{0}}$ & Gas temperature & $\mathrm{K}$ & 297 \\
$\mu_{g_{0}}$ & Gas viscosity & $\mathrm{g} / \mathrm{cm} / \mathrm{s}$ & $1.8 e^{-4}$ \\
$t_{\text {start }}$ & Start time & $\mathrm{S}$ & 0.2 \\
$t_{\text {stop }}$ & Stop time & $\mathrm{s}$ & 1.0 \\
$\rho_{s}$ & Particle density & $\mathrm{g} / \mathrm{cm}{ }^{3}$ & 2.61 \\
$D_{p}$ & Particle diameter & $\mathrm{cm}$ & 0.05 \\
$h_{s 0}$ & Initial height of packed bed & $\mathrm{cm}$ & 29.22 \\
$\epsilon_{g}^{*}$ & Initial void fraction of packed bed & - & 0.4 \\
\hline
\end{tabular}

bubbles, the discontinuities in the low-energy bubbles are extremely weak and we expect the normal POD modes to accurately capture them.

Mathematical morphology was shown to extract key features from an image by the use of a simple set of operations. Regardless of the original grid arrangement of the data, the morphological discontinuity detection algorithm presented has the ability to detect the high energy bubbles present in multiphase flows that lead to the Gibbs phenomenon. The discontinuities are detected with two-point accuracy.

\section{Conclusions}

All of the project deliverables have been achieved. Reduced-order models for the solids volume fraction correction equation, the energy balance equations and the granular energy equation have been implemented in ODEx. A reduced-order model for the $z$-direction conservation of gas and solids momentum has also been implemented and the other algorithms in ODEx have been modified to include the fluxes through the top and bottom of the computational cells, allowing the simulation of three-dimensional flow.

MFIX was used to build snapshot databases for a variety of simple test cases and the POD basis functions were computed for each case using PODDEC. These cases were then used to verify and validate the ODEx computations. In general, the agreement between ODEx and MFIX was very good.

A User's Manual and a Theory Guide were written to facilitate the use of ODEx. The 


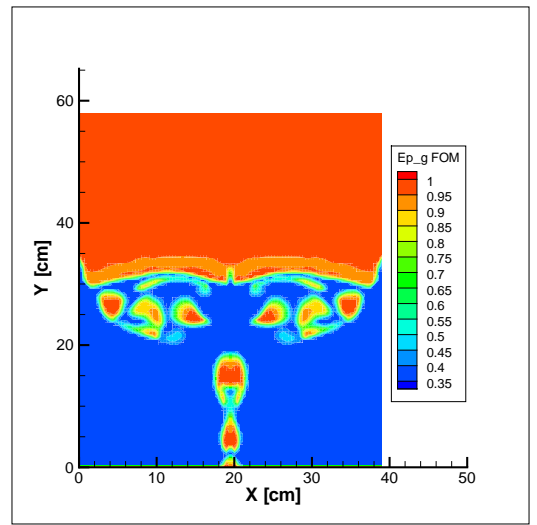

(a)

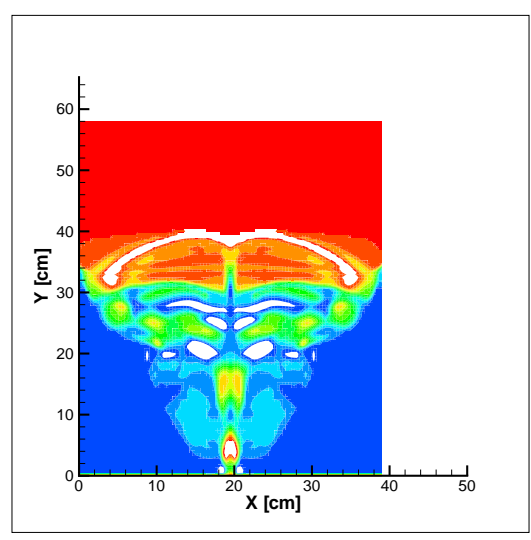

(d)

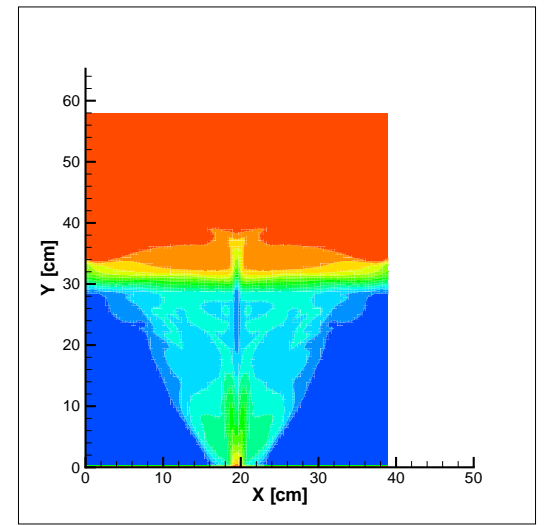

(b)

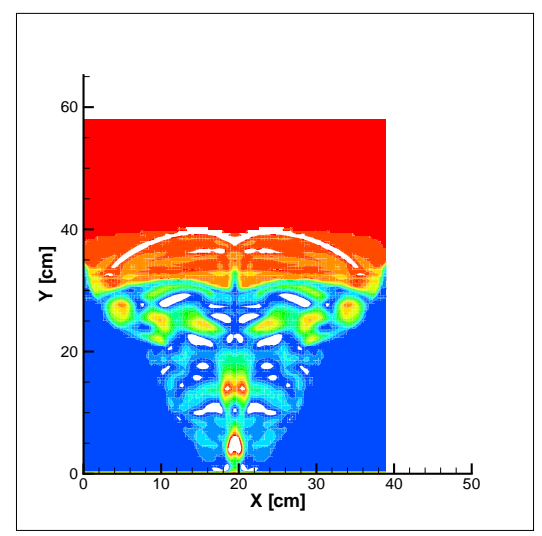

(e)

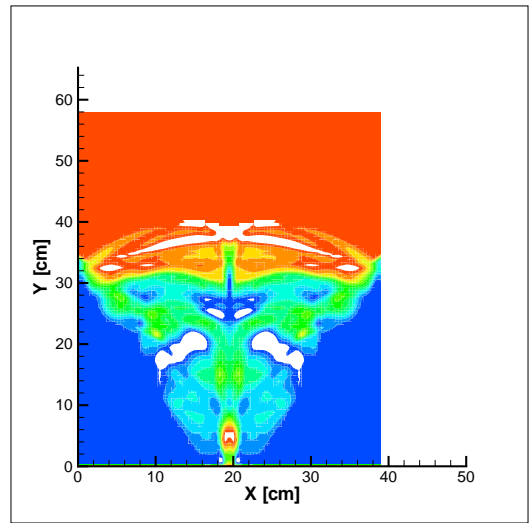

(c)

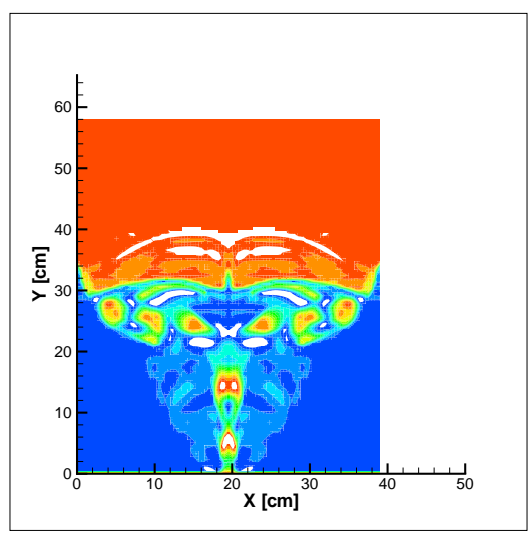

(f)

Figure 38: Contour plots for void fraction at $t=1.0 \mathrm{~s}$ : (a) full-order model; (b) POD reconstruction with 4 modes; (c) POD reconstruction with 8 modes; (d) POD reconstruction with 12 modes; (e) POD reconstruction with 16 modes; (f) POD reconstruction with 20 modes.

early results of the research conducted during this project have been published in archival journals. The remaining results will be published in the following three months. Additionally, the interfaces needed for the CAPE-OPEN standard have been identified.

Moving discontinuities in the void fraction variable exist in multiphase flow in the form of bubbles. Two methods have been developed to address the unphysical approximation produced by POD-based ROMs near moving discontinuities. These methods have been implemented and tested for simple one-dimensional and two-dimensional canonical cases, such as the wave equation and Burgers equation. The proposed adaptations include an augmented POD method and a point-mode method. These methods have not been yet implemented in the ODEx code. 

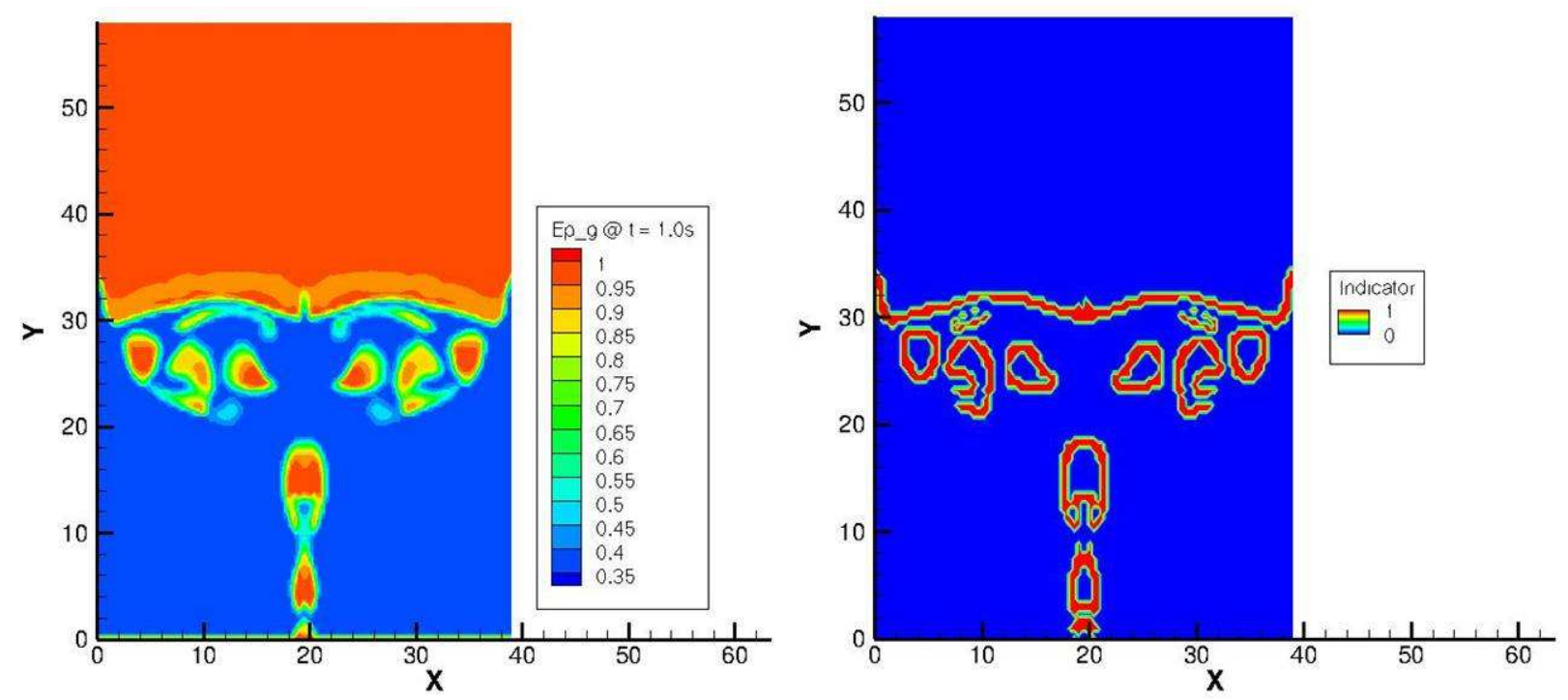

Figure 39: Results of the application of the shock detection algorithm to a bubbling fluidized bed. (a) is the bubbling fluidized bed's void fraction $\epsilon_{g}$ at $\mathrm{t}=1.0 \mathrm{~s}$. (b) is the edge indicator of (a) after thresholding.

\section{Future Work}

The difficulties inherent in modeling bubbling flow with a POD based ROM represent a significant potential source for future research. This report has summarized two methods for adapting the set of basis functions to better capture moving discontinuities. These are, however, by no means the only approaches available to solve this problem.

Another potential approach is to compute POD basis functions for the solids volume fraction correction. Then the solids volume fraction would be the value at a previous subiteration, plus the approximate correction. This would mean that the solids volume fraction has converged to the correct value as the value of the time coefficients associated with the basis functions approach zero. This approach would also allow the more efficient implementation of the point-mode method.

Yet another approach is to build a reduced-order model for the integral form of the conservation of gas and solids mass equations. The POD approximation of the gas pressure and void fraction could then be substituted and the Galerkin projection taken. This would result in a general set of ordinary differential equations that are suited to any finite volume discretization scheme and allow easy implementation of a point-mode method. 


\section{References}

Benyahia, S., M. Syamlal, and T. J. O'Brien (2007, July). Summary of MFIX Equations 2005-4. NETL. http://www.mfix.org/documentation/MfixEquations2005-4-3.pdf.

Brenner, T. A., P. G. A. Cizmas, T. J. O’Brien, and R. W. Breault (2009, January). Practical aspects of the implemenation of proper orthogonal decomposition. In 4 'th AIAA Aerospace Sciences Meeting, Orlando, FL.

Cizmas, P. G. A. and A. Palacios (2003, March-April). Proper orthogonal decomposition of turbine rotor-stator interaction. AIAA Journal of Propulsion and Power 19(2), 268-281.

Cizmas, P. G. A., B. R. Richardson, T. A. Brenner, T. J. O'Brien, and R. W. Breault (2008). Acceleration techniques for reduced-order models based on proper orthogonal decomposition. Journal of Computational Physics 227, 7791-7812.

CO-LaN consortium (2003, August). Methods \& Tools Integrated Guidelines (1 ed.). COLaN. http://www.colan.org/Spec\%2010/Documentation\%20set\%201.0.zip.

Fehlberg, E. (1969). Low-order classical Runge-Kutta formulas with stepsize control and their application to some heat transfer problems. Technical report, NASA, USA.

Gidaspow, D. (1994). Multiphase Flow and Fluidization. Boston: Academic Press.

Holmes, P., J. L. Lumley, and G. Berkooz (1996). Turbulence, Coherent Structures, Dynamical Systems and Symmetry. Cambridge University Press.

Kirby, M., J. P. Boris, and L. Sirovich (1990). A proper orthogonal decomposition of a simulated supersonic shear layer. International Journal for Numerical Methods in Fluids 10, 411-428.

Lee, J. S. J., R. Haralick, and L. Shapiro (1987, April). Morphological edge detection. IEEE J. of Robotics and Automation RA-3(2), 142-156.

Lucia, D. J., P. I. King, and P. S. Beran (2003). Reduced order modeling of a two-dimensional flow with moving shocks. Computers $\&$ Fluids 32, 917-938.

Press, W. H., W. T. Vetterling, S. A. Teukolsky, and B. P. Flannery (1992). Numerical Recipes in FORTRAN - The Art of Scientific Computing (Second ed.). Cambridge.

Richardson, B. R. (2007, December). A reduced-order model based on proper orthogonal decomposition for two-phase flow non-isothermal transport phenomena. Master's thesis, Texas A\&M University, College Station, TX.

Ritter, G. and J. Wilson (2000). Computer Vision Algorithms in Image Algebra (2nd ed.)., Chapter 4, pp. 137-138. CRC Press. 
Sirovich, L. (1987). Turbulence and the dynamics of coherent structures. Quarterly of Applied Mathematics 45(3), 561-590.

Soille, P. (2003). Morphological Image Analysis: Principles and Applications (2nd ed.). Springer-Verlag.

Syamlal, M. (1998). MFIX documentation numerical technique. Technical Report DE-AC2195MC31346, EG\&G Technical Services of West Virginia.

Syamlal, M., W. Rogers, and T. J. O'Brien (1994). MFIX documentation theory guide. Technical Report DOE/METC-94/1004, DOE/METC.

Yuan, T. (2003, December). Reduced order modeling for transport phenomena based on proper orthogonal decomposition. Master's thesis, Texas A\&M University, College Station, Texas.

Yuan, T., P. G. Cizmas, and T. O'Brien (2005). A reduced-order model for a bubbling fluidized bed based on proper orthogonal decomposition. Computers and Chemical Engineering 30(2), 243-259. 